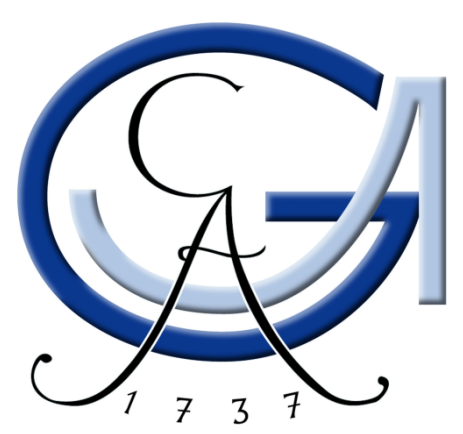

\title{
ROBUST ELECTROMYOGRAPHY BASED CONTROL OF MULTIFUNCTIONAL PROSTHESES OF THE UPPER EXTREMITY
}

\author{
Dissertation \\ zur Erlangung des mathematisch-naturwissenschaftlichen \\ Doktorgrades \\ "Doctor rerum naturalium" \\ der Georg-August-Universität Göttingen \\ im Promotionsprogramm PCS \\ der Georg-August University School of Science (GAUSS) \\ vorgelegt von \\ Sebastian Amsüss, MSc.
}

aus Wien

Göttingen, 2014 


\section{Betreuungsausschuss:}

Referent: Prof. Dr. Florentin Wörgötter, Dept. of Computational Neuroscience

Koreferent: $\quad$ Prof. DDr. Dario Farina, Dept. of Neurorehabilitation Engineering

Externer Betreuer Priv. Doz. Dr. Bernhard Graimann, Otto Bock HealthCare GmbH

\section{Weitere Mitglieder der Prüfungskommission:}

Prof. Dr. Otto Rienhoff

Prof. Dr. Ulrich Sax

Prof. Dr. Hansjörg Scherberger

Prof. Dr. Dieter Hogrefe

Tag der mündlichen Prüfung: 26. September 2014 


\section{Preface And Declarations}

The results presented in this thesis were obtained as part of my work at the Department of Neurorehabilitation Engineering at the University Medical Center Göttingen. My work was funded by the European Commission via the IAPP project AMYO, project reference 251555, project duration September $1^{\text {st }} 2010$ until August $31^{\text {st }} 2014$.

All studies involving human subjects were approved by the local ethics committee and all subjects signed informed consents prior to their participation in the studies.

All parts of this thesis are originally written by me. Partially, some of the material has also been published by me as the principal author or as co-author in other publications. The relevant references are cited herein adhering to scientific practice. All rights for re-use of previously published material were obtained. Reused figures and tables of IEEE publications are marked with (C)[Year] IEEE.

Hereby I declare that I have written this thesis independently and with no other aids and sources than quoted.

Göttingen, September 13, 2014

Sebastian Amsüss 


\section{Abstract}

Multifunctional, highly dexterous and complex mechanic hand prostheses are emerging and currently entering the market. However, the bottleneck to fully exploiting all capabilities of these mechatronic devices, and to making all available functions controllable reliably and intuitively by the users, remains a considerable challenge. The robustness of scientific methods proposed to overcome this barrier is a crucial factor for their future commercial success.

Therefore, in this thesis the matter of robust, multifunctional and dexterous control of prostheses of the upper limb was addressed and some significant advancements in the scientific field were aspired. To this end, several investigations grouped in four studies were conducted, all with the same focus on understanding mechanisms that influence the robustness of myoelectric control and resolving their deteriorating effects.

For the first study, a thorough literature review of the field was conducted and it was revealed that many non-stationarities, which could be expected to affect the reliability of surface EMG pattern recognition myoprosthesis control, had been identified and studied previously. However, one significant factor had not been addressed to a sufficient extent: the effect of long-term usage and day-to-day testing. Therefore, a dedicated study was designed and carried out, in order to address the previously unanswered question of how reliable surface electromyography pattern recognition was across days. Eleven subjects, involving both able-bodied and amputees, participated in this study over the course of 5 days, and a pattern recognition system was tested without daily retraining. As the main result of this study, it was revealed that the time between training and testing a classifier was indeed a very relevant factor influencing the classification accuracy. More estimation errors were observed as more time lay between the classifier training and testing.

With the insights obtained from the first study, the need for compensating signal non-stationarities was identified. Hence, in a second study, building upon the data obtained from the first investigation, a self-correction mechanism was elaborated. The goal of this approach was to increase the systems robustness towards non-stationarities such as those identified in the first study. The system was capable of detecting and correcting its own mistakes, yielding a better estimation of movements than the un- 
corrected classification or other, previously proposed strategies for error removal. In the third part of this thesis, the previously investigated ideas for error suppression for increased robustness of a classification based system were extended to regression based movement estimation. While the same method as tested in the second study was not directly applicable to regression, the same underlying idea was used for developing a novel proportional estimator. It was validated in online tests, with the control of physical prostheses by able-bodied and transradial amputee subjects. The proposed method, based on common spatial patterns, outperformed two state-of-the art control methods, demonstrating the benefit of increased robustness in movement estimation during applied tasks. The results showed the superior performance of robust movement estimation in real life investigations, which would have hardly been observable in offline or abstract cursor control tests, underlining the importance of tests with physical prostheses.

In the last part of this work, the limitation of sequential movements of the previously explored system was addressed and a methodology for enhancing the system with simultaneous and proportional control was developed. As a result of these efforts, a system robust, natural and fluent in its movements was conceived. Again, online control tests of physical prostheses were performed by able-bodied and amputee subjects, and the novel system proved to outperform the sequential controller of the third study of this thesis, yielding the best control technique tested.

An extensive set of tests was conducted with both able-bodied and amputee subjects, in scenarios close to clinical routine. Custom prosthetic sockets were manufactured for all subjects, allowing for experimental control of multifunction prostheses with advanced machine learning based algorithms in real-life scenarios. The tests involved grasping and manipulating objects, in ways as they are often encountered in everyday living. Similar investigations had not been conducted before. One of the main conclusions of this thesis was that the suppression of wrong prosthetic motions was a key factor for robust prosthesis control and that simultaneous wrist control was a beneficial asset especially for experienced users. As a result of all investigations performed, clinically relevant conclusions were drawn from these tests, maximizing the impact of the developed systems on potential future commercialization of the newly conceived control methods. This was emphasized by the close collaboration with Otto Bock as an industrial partner of the AMYO project and hence this work. 


\section{ACKNOWLEDGMENTS}

First and foremost, I would like to express my thankfulness to Prof. Dr. Dario Farina, who offered me better guidance than I could have hoped for during the course of my work. His exceptional professional expertise in just about everything as well as his kind and gentle personal being have inspired me throughout my time in his department - and will certainly continue so in the furture.

Further I would like to thank Prof. Dr. Florentin Wörgötter, without whom I would not have been able to complete this work. I valued his support in all matters regarding this thesis - from its initial phase until its finalization.

I thank PD. Dr. Bernhard Graimann from Otto Bock HealthCare GmbH for his management of the AMYO project, which allowed me to pursue my Ph.D. program as early stage researcher at the University Medical Center Göttingen. Thank you also to Peter Michael Göbel from Otto Bock Healthcare Products GmbH for the close and fruitful collaboration over the years.

Moreover, I thank the entire Department of Neurorehabilitation Engineering led by Prof. Farina, which I was lucky enough to be part of. I cherished the various movie nights, gaming nights and barbecues we all shared together. Particularly I would like to thank my office (snacks) colleague Jakob. Thanks to him, help was always just a chair-spin away. I enjoyed sharing work and leisure time with him, as well as with all the colleagues I have met here.

Furthermore I thank all subjects who volunteered for their participation in the experiments conducted within this thesis.

Last, but certainly not least, I embrace my entire family for their endorsement during the years I spent away from home, their constant encouragement and their never ending support.

For Elise, my completion. "Meilleure équipe du monde, toujours" 


\section{Contents}

1 Introduction 1

1.1 Limitations of current myoelectric control . . . . . . . . . . . . . 2

1.2 State of the art hardware in transradial prostheses . . . . . . . . . . . 3

1.3 The early days of myoelectric pattern recognition . . . . . . . . . . . 3

1.4 Extracting more information from the EMG . . . . . . . . . . . . 4

1.5 Estimators . . . . . . . . . . . . . . . . . 6

1.5.1 Classifiers - choosing either-or . . . . . . . . . . 6

1.5.2 Regressors - It doesn't always have to be either-or . . . . . . . 12

1.6 Current state of the art . . . . . . . . . . . . . . . . . . . 14

1.7 Thesis goal and outline . . . . . . . . . . . . . . . . . . 15

2 Time related robustness $\quad 17$

2.1 Methods and procedures . . . . . . . . . . . . . . . 18

2.1.1 Test protocol . . . . . . . . . . . . . . . . 20

2.1.2 Signal processing . . . . . . . . . . . . . . . . . . 21

2.2 Results . . . . . . . . . . . . . . . . . . . . . . 21

2.3 Study discussion and conclusion . . . . . . . . . . . . . 26

3 Self correcting classification system $\quad 29$

3.1 Methods and procedures . . . . . . . . . . . . . . 31

3.1.1 Self correction system _ . . . . . . . . . . . . . 32

3.1.2 Implementation of self-correction mechanism . . . . . . . . . . 33

3.1 .3 Evaluations . . . . . . . . . . . . . . . . . . 36

3.1.4 Statistical Analysis . . . . . . . . . . . . . . . . 37

3.2 Results . . . . . . . . . . . . . . . . . . 37

3.2.1 Able-bodied subjects . . . . . . . . . . . . . 40

3.2.2 Amputee subjects .................. 43 
3.2 .3 Time accuracy . . . . . . . . . . . . . . . . . . 44

3.3 Study discussion and conclusion . . . . . . . . . . . . . 45

4 A novel multi-class proportional estimator 49

4.1 Methods and procedures . . . . . . . . . . . . 50

4.1.1 Common spatial patterns proportional estimator (CSP-PE) . . 51

4.1 .2 Compared methods . . . . . . . . . . . . . . 56

$4.1 .3 \quad$ Subjects . . . . . . . . . . . . . . . . . . . 58

4.1 .4 Applied test scenarios $\ldots \ldots \ldots \ldots$

4.1 .5 Questionnaire . . . . . . . . . . . . . . . . 63

4.1.6 Hardware control system . . . . . . . . . . . . . . . 63

4.1 .7 Data acquisition . . . . . . . . . . . . . . . . 64

4.1 .8 Statistical analysis . . . . . . . . . . . . . . . 64

4.2 Results . . . . . . . . . . . . . . . . . . . . 65

4.2 .1 Able-bodied subjects . . . . . . . . . . . . . 65

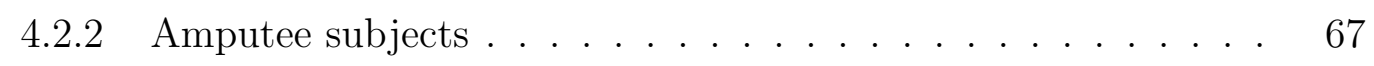

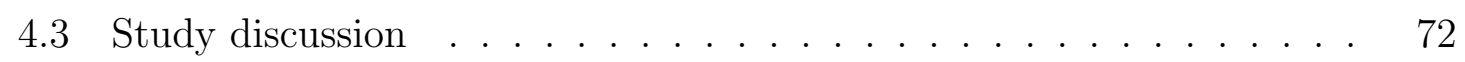

4.3 .1 Machine learning methods . . . . . . . . . . . . . . 72

4.3.2 Extended state-of-the-art control . . . . . . . . . . . . 73

4.3 .3 Block turn test . . . . . . . . . . . . . . . . 73

4.4 Study conclusion . . . . . . . . . . . . . . . . . 74

5 Combining sequential and simultaneous regressors $\quad 75$

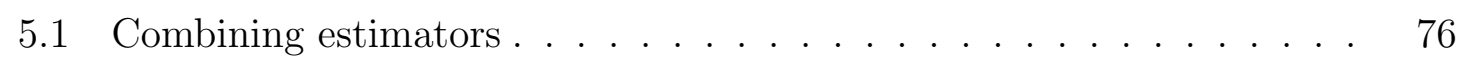

5.1.1 Embedded dimensionality estimators . . . . . . . . 76

5.1 .2 Methods for identifying the optimal EDE . . . . . . . . 80

5.1 .3 Statistical Analysis . . . . . . . . . . . . . . . 81

5.1.4 Results of EDE performance . . . . . . . . . . . . . 81

5.2 Online control of physical prostheses $\ldots \ldots \ldots \ldots \ldots$

5.3 Results. . . . . . . . . . . . . . . . . . . . 85

5.3 .1 Amputee SHAP results . . . . . . . . . . . . . 85

$5.3 .2 \quad$ Able-bodied results . . . . . . . . . . . . . . . . . . . 89

5.4 Study discussion and conclusion $\ldots \ldots \ldots \ldots \ldots$

6 Thesis discussion and conclusion $\quad 95$ 
7.1 List of publications . . . . . . . . . . . . . . . . . . . . 115

7.2 Reviewer activities . . . . . . . . . . . . . . . 116 


\section{GLOSSARY}

a/tAcc active/total accuracy

ADL activities of daily living

ANN artificial neural network

CSP common spatial patterns

CSP-PE CSP proportional estimator

CSP-PE+LR combination of CSP-PE and LR

DOF degree of freedom

EDE embedded dimensionality estimators

i/s-EMG intramuscular/surface electromyogram

FP fine pinch

HO hand opening

KG key grip

KNFST Kernel Null Foley-Sammon Transform

kNN k-Nearest Neighbor

LDA linear discriminant analysis

LR linear regression

MD Mahalanobis distance

NM no movement

OCSVM one class support vector machine

RMS root mean square

SHAP Southampton hand assessment procedure

SOA state of the art

SVM support vector machine

TI trust index

WE wrist extension

WF wrist flexion

WP wrist pronation

WS wrist supination 


\section{Introduction}

The importance of our hands is appreciated in all daily tasks - from object manipulation to communication, gesticulation, touching, feeling, caressing and holding hands with a loved-one. The vast variety of actions our hands are involved in makes us understand the large impact on the quality of life of not having them. In 2008 it was estimated that 41,000 persons with major upper limb amputation (above finger level) were living in the United States alone [1] and 31\% of all upper limb amputation procedures were performed at transradial level [2]. In total, around $16 \%$ of all upper limb amputations occur at higher than finger level [3]. The majority of upper limb amputations are secondary to traumatic incidences (estimated around $83 \%$ in 2005 in the U.S.) followed by dysvascular diseases (12\%) and oncological etiologies (5\%) [1]. These numbers show representative estimates for the U.S. where most substantial surveys have been published in literature originate from. It is difficult to generalize from these estimates to the entire population worldwide. In developing countries and regions afflicted by war, the prevalence of upper limb absence might be significantly higher [4].

The presented numbers of persons with upper limb deficiency coupled with the severe consequences of arm loss put a high demand and expectations towards reconstructive measures for such handicaps. While the transplantation of hands and arms has recently been proven to be a possibility for alleviating the severity of upper extremity amputation [5], prosthetic devices are still the preferred way of treatment. A variety of prosthetic systems is available on the market, ranging from purely cosmetic prostheses to multifunctional, externally powered and myoelectric controlled systems.

The most life-like and natural, non-fatiguing control is offered by myoelectric prostheses and is thus regarded as the state of the art in prosthetic devices [6]. The control of this type of prosthesis relies on the lead of electrical potentials which originate from neuromuscular discharges during muscle activations [7]. For prosthetic applications, 
these signals are usually measured as electromyographic (EMG) signals on the skin surface (sEMG), however also implantable sensors (iEMG) are being investigated in experimental research settings $[8,9,10,11]$. The benefits of surface electrodes consist in their easy applicability, negligible risks for the wearer, minimal invasiveness and the possibility to integrate them in the prosthetic sockets. For these reasons, surface electrodes are exclusively used in commercial state-of-the-art myoelectric controlled prostheses. However, certain drawbacks of this methodology exist and a need for improvement of the current control strategies is desirable, as will be motivated in detail in the following sections.

\subsection{Limitations of current myoelectric control}

In commonly commercially available myoelectric prostheses a maximum of two sEMG electrodes is used. These are strategically placed over regions that exhibit maximal and most distinctive activations of the remnant muscles during phantom limb movements of the amputee $[12,13]$. It is up to the orthopedic technician fitting the prosthesis to the wearer to identify these optimal placements, which often proves to be a bottleneck in the prosthetic care [14]. In the case that two independently controllable muscle sites with sufficient sEMG signal quality can be found, a 1-to-1 mapping between electrodes and prosthetic functions is performed. The global activity of the underlying muscle group beneath one electrode is estimated, usually by calculating the signal power or envelope, and this estimate is used to drive one function of the prosthesis. In most cases, the best configuration is obtained by placing one electrode above the wrist flexor and one above the wrist extension muscles. Closing the prosthetic prehensor is then mapped to the flexor electrode while opening to the extensor. In the event that not enough sites with sufficient signal quality can be found by the clinicians, only one electrode may be used and the two prosthetic control functions are obtained by distinguishing between slow and fast or low and high contractions in order to obtain the desired two function control [15,16]. Experienced users may even use the latter strategy with two electrodes, resulting in the so-called four channel control [17], allowing to address four prosthetic functions with two sEMG signals. This is however only applicable for amputees with excellent signal quality, precise proportional control and requires extensive training. Thus, more commonly, a switching paradigm is adopted for the control of more than 2 functions. Either a hardware 
switch integrated in the prosthetic socket [17] or a special muscle signal is used for this purpose. In the latter case, the most common choice is the use of co-contractions, elicited by a quick and short contraction of both the flexor and extensor muscles. This scheme has proven to be very robust in practice, however its extensibility to more than four functions is questionable although it has not been studied in detail previously. In Chapter 4 this topic will be addressed in a comparative study.

In conclusion, the classic two channel control, which is the state of the art in myoelectric controlled prostheses, is reliable and robust, however the number of controllable functions is very limited.

\subsection{State of the art hardware in transradial pros- theses}

The first externally powered hand prostheses were developed in the 1940s $[18,19]$ and refined and commercialized 15 years later [20,21]. Ever since, the rapid advances of microelectronics, electric actuators and battery technologies drastically propelled the further developments of these devices. To date, a large variety of multifunctional hand prostheses with many degrees of freedom is available commercially and for research, mimicking their natural anatomical counterparts in appearance, force, precision and dexterity. In a recent review [22], an overview over the various types and specifications of the most relevant hand prostheses was given. The common trend in all these modern devices is the increasing number of actuated joints, allowing for complex movements, natural in appearance. As discussed in Section 1.1 however, there is a substantial disparity between the number of actuated motions in these devices and the number of control signals that can be obtained with the conventional control strategies.

\subsection{The early days of myoelectric pattern recogni- tion}

Researchers therefore began to explore alternative signal processing techniques, allowing for the control of several prosthetic movements. Soon, the domain of machine learning in general and pattern recognition in particular were identified as suitable 
candidates for accomplishing this challenging task. The first work in this direction was published in 1967 by Finley and Wirta [23] and soon pursued by other groups [24,25]. The work of Herberts, Almström and Caine [25] published in 1978, in which a wearable prosthesis implementing wrist rotation, wrist flexion and extension and hand opening and closing controlled by pattern recognition of sEMG signals was developed, is particularly interesting. The pattern recognition system used was discriminant analysis and was implemented in hardware with analogue circuits. The system was tested in four amputees. Two evaluation schemes involving abstract computer tests and activities of daily living (ADL) had to be completed by the subjects.

Apart from relatively small changes, this study design would still comply with current state of the art research standards, which is rather surprising considering that in the meantime almost four decades have passed.

\subsection{Extracting more information from the EMG}

A significant improvement to the above described system was introduced in 1993 by Hudgins, Parker and Scott [26]. In their contribution they suggested to extract more features from the EMG signal than only the global muscle activation. By windowing and interlacing those windows, the EMG signal was regarded as quasi-stationary over short periods of time $(<300 \mathrm{~ms})$, and certain characteristic features could be extracted with sufficient repeatability across windows [27]. Those features comprised the mean absolute value (MAV), the number of signal zero crossings (ZC), slope sign changes (SSC) and the global wave form length (WL): (adapted from [26])

$$
\begin{aligned}
& M A V:=\frac{1}{N} \sum_{k=1}^{N}\left|x_{k}\right| \\
& Z C:=\frac{1}{N} \sum_{k=1}^{N} c, c= \begin{cases}1, & \text { if } x_{k} \cdot x_{k+1}<0 . \\
0, & \text { otherwise or if }\left|x_{k}-x_{k+1}\right|<0.01 V .\end{cases} \\
& S S C:=\frac{1}{N} \sum_{k=1}^{N} c, c= \begin{cases}1, & \text { if } x_{k} \gtrless x_{k-1} \text { and } x_{k} \gtrless x_{k+1} . \\
0, & \text { otherwise or if }\left|x_{k}-x_{k+1}\right|<0.01 \mathrm{~V} .\end{cases} \\
& W L:=\frac{1}{N} \sum_{k-1}^{N}\left|x_{k}-x_{k-1}\right|
\end{aligned}
$$


where $x_{k}$ is the $k^{t h}$ sample of the current time window with $N$ samples. For the calculation of the ZC and SSC features, a threshold between two adjacent samples of $0.01 \mathrm{~V}$ (corresponding to $4 \mu \mathrm{V}$ peak to peak raw signal) was proposed to be included in order to make these features less affected by random noise. The MAV value contained information related to the amplitude of the EMG signal, the ZC and SSC were simple frequency measures (for lower and higher frequencies, respectively) and the WL contained mixed information of amplitude and frequency. These features were extracted from every EMG channel and are still widely used and regarded as the "standard" feature set in myoelectric pattern recognition, termed Hudgins time domain features (see for example [28] for an exemplary overview of studies which used this set of features for myoelectric prosthesis control). Sometimes, the MAV is replaced by the root mean square (RMS) value of the signal, defined as

$$
R M S:=\sqrt{\frac{1}{N} \sum_{k=1}^{N}\left(x_{k}\right)^{2}}
$$

The basic idea of splitting the EMG in interlaced windows and calculating features from these signal segments is still the most widely used. Over the years, the Hudgins time domain feature set has been substantially expanded, by other features computed in the time domain $[29,30]$, frequency domain and time-frequency domain features, such as extracted by short time fast Fourier transform (STFFT) and wavelet transformation (WT) [31,32, 33, 34, 35, 36], autoregressive models [29], fractal dimension [37] and several more.

\section{Importance of the feature set}

The features extracted from the EMG signals constitute the basis for separability of different movements by EMG. The desired characteristic of the optimal features is to represent as distant as possible and with minimal intra-class variability the different motions to be classified. This was first analyzed in [29], where the Davis-Bouldin cluster separation measure (DBCSM) was used to evaluate a variety of features precisely regarding these qualities. DBCSM is related to Fisher's criterion of separability [38,39], which is also used as the optimization criterion in one of the most popular classifiers (see Section 1.5.1). In their evaluations, Zardoshti-Kermani et al. found that the time domain features differed in their importance for separability and that the EMG histogram feature performed best, followed by WL and the amplitude 
related features [29]. Oskoei and $\mathrm{Hu}$ [34] found that time domain features yielded slightly better classification accuracy when compared to features obtained in the frequency domain. The best classification accuracies were reported for mixed feature sets, for example time domain and autoregressive features [9, 40, 41, 42, 43].

From these findings reported in literature, the discrepancy in different feature sets in general appears small. Complex features often require hyper parameter selection or optimization, substantially increased computation time and their sensitivity to noise and electrode shifts is largely unknown (as opposed to time domain features which have been studied extensively in that respect, see e.g. [44]). Therefore, the simple time domain features offer a very competitive option and yield the best trade-off between simplicity and performance.

\subsection{Estimators}

With the most relevant features extracted from the EMG signals, the next step in the signal processing chain (Figure 1.1) is to translate the captured information to movement commands. A long list of machine learning methods provides a series of estimators to accomplish this task. The methods can be generally grouped into classification and regression approaches. The former yield discrete outputs used as class labels. The latter fit smooth curves to e.g. force functions. In the following, the most relevant methods, representatives of each type, are briefly presented and discussed.

\subsubsection{Classifiers - choosing either-or}

In the first studies using pattern recognition for myoelectric prosthetic control (refer to Section 1.3), discriminant analysis was used. Later, artificial neural networks (ANN) were introduced [26] and extensively used (e.g. [43, 45, 46]). Further popular choices for non-parametric classifiers are for example k-nearest neighbor $(\mathrm{kNN})[29,47]$ and support vector machines (SVM) $[31,34,48,49]$, whereas linear and quadratic discriminant analysis (LDA, QDA), Gaussian mixture models and hidden markov models are some investigated examples for parametric classifiers. For an extensive comparison of different features, in combination with different estimators applied to sEMG and iEMG signals, the interested reader is referred to [50]. Further comparisons of different classifiers are found in $[28,47,51]$. 


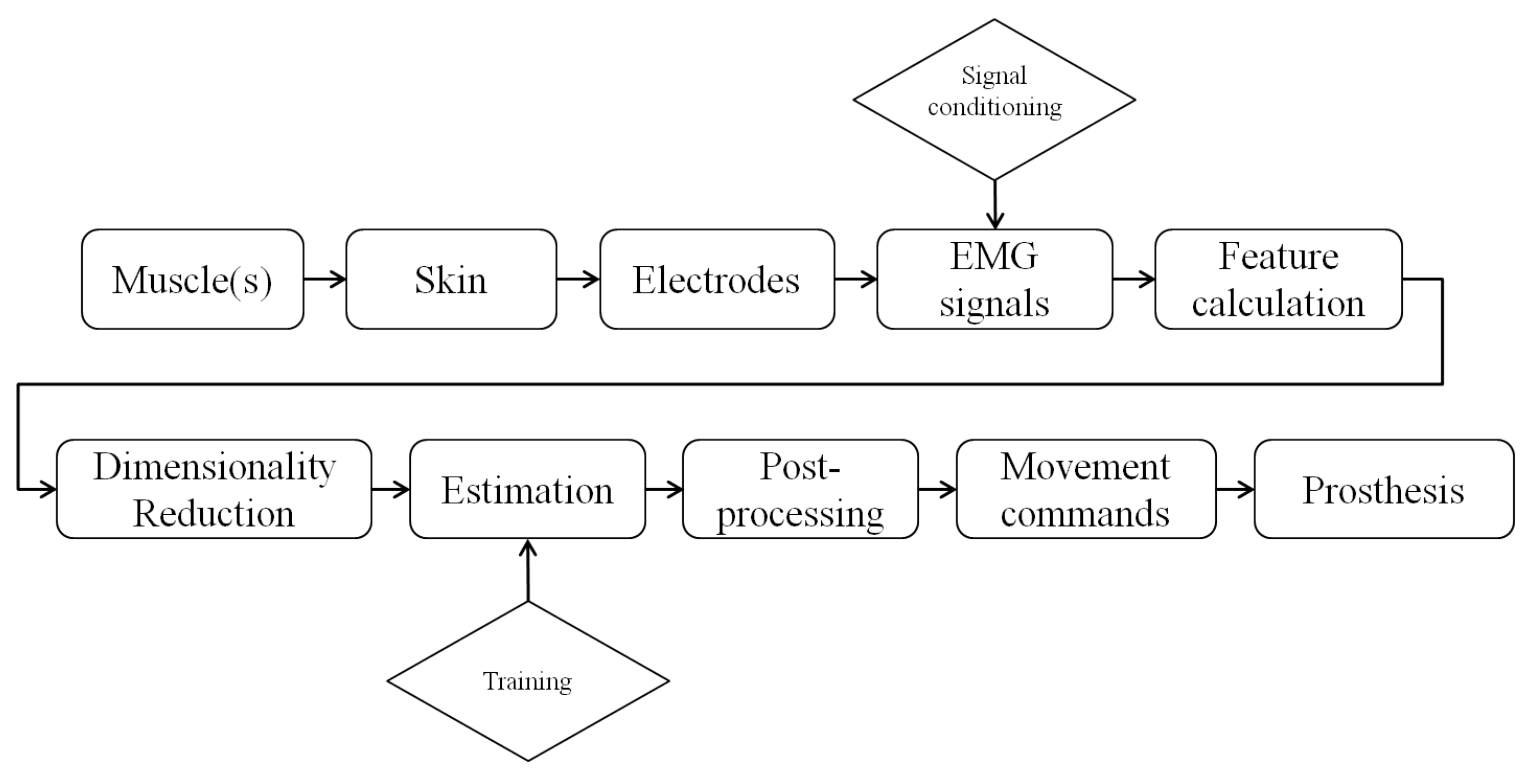

Figure 1.1: Typical signal processing chain of a modern myoelectric control pattern recognition system for upper limb prostheses. The signals originate in the muscle fibers, propagate through the arm tissue to the skin where they are picked up as sEMG signals. The signals are filtered, amplified and digitized. After windowing, discriminative signal features are calculated. In case of large resulting dimensionality (many sEMG channels, many features), dimensionality reduction is performed prior to calculating an estimate of the performed movement. The estimator needs to be trained with a series of training data. The raw estimation outputs are postprocessed (e.g. again windowed, filtered...) and ultimately the prosthesis control commands are sent to the prosthetic control unit driving the actuators. 
As for the features, in general, simple and computationally cheap, (hyper-)parameter independent, well studied and robust classifiers yield comparable results to more complex and sensitive methods and are therefore the methods of choice in a generic setup. These classifiers were also preferred in this work. However, as will be discussed in Chapter 4, significant performance improvements can be achieved by specific, targeted modifications of existing methods for desired objectives.

\section{Linear Discriminant Analysis (LDA) classifier}

In this section the LDA classifier is introduced in detail, since it will be used extensively in the further progress of this thesis as well as other related algorithms, such as CSP, PCA and KNFST (see later chapters for details on these methods). LDA attempts to express the dependent variable (class) by a linear combination of independent variables (features). This section has been adapted and extended from $[38,39]$. LDA is closely related to the Fisher discriminant ratio (FDR), given as

$$
F D R=\frac{\left(\mu_{1}-\mu_{2}\right)^{2}}{\sigma_{1}^{2}+\sigma_{2}^{2}}
$$

where $\mu_{i}$ and $\sigma_{i}^{2}$ are the class means and variances in the transformed space, respectively. The criterion thus optimizes the feature separability (minimal inter-class dispersion, maximal between-class dispersion) in the transformed space, resulting in an optimized setting for classification. Realizing that the variance of variable $y$ transformed from an input vector $x$ with the linear projection vector $w$

$$
y=\boldsymbol{w}^{T} \boldsymbol{x}+w_{0}
$$

(1.6) can be obtained in the transformed space from the input space by

$$
F D R(\boldsymbol{w})=\frac{\boldsymbol{w}^{T} \Sigma_{b} \boldsymbol{w}}{\boldsymbol{w}^{T} \Sigma_{w} \boldsymbol{w}}
$$

where $\Sigma_{b}$ is the covariance matrix between the class means of the different classes and $\Sigma_{w}$ is the average covariance matrix of data belonging to the same class.

In order to maximize the separability criterion given in (1.8), $\boldsymbol{w}$ has to be chosen such that $F D R(\boldsymbol{w})$ is maximized:

$$
\underset{\boldsymbol{w}}{\arg \max } F D R(\boldsymbol{w})=\underset{\boldsymbol{w}}{\arg \max } \frac{\boldsymbol{w}^{T} \Sigma_{b} \boldsymbol{w}}{\boldsymbol{w}^{T} \Sigma_{w} \boldsymbol{w}}
$$


To make the problem well defined, the scaling factor of $\boldsymbol{w}$ has to be fixed, which can be achieved by setting the norm of $\boldsymbol{w}$ to 1 : $\|\boldsymbol{w}\|^{2}=\boldsymbol{w}^{T} \boldsymbol{w}=1$.

This results in the constrained optimization problem:

$$
\underset{\boldsymbol{w}}{\arg \max } \frac{\boldsymbol{w}^{T} \sum_{b} \boldsymbol{w}}{\boldsymbol{w}^{T} \sum_{w} \boldsymbol{w}} \text { subject to: } \boldsymbol{w}^{T} \boldsymbol{w}=1
$$

Eq. (1.10) is a standard mathematical problem and is known as quadratic programming. The standard technique yielding a closed form solution for such a problem is by transforming the problem to a Lagrangian formulation $\mathcal{L}(\boldsymbol{w})$

$$
\mathcal{L}(\boldsymbol{w})=\frac{\boldsymbol{w}^{T} \Sigma_{b} \boldsymbol{w}}{\boldsymbol{w}^{T} \Sigma_{w} \boldsymbol{w}}-\lambda\left(\boldsymbol{w}^{T} \boldsymbol{w}-1\right)
$$

where $\lambda$ are the Lagrange multipliers. Differentiating (1.11) w.r.t. $\boldsymbol{w}$ and setting to 0 :

$$
\begin{aligned}
\frac{\partial \mathcal{L}(\boldsymbol{w})}{\partial \boldsymbol{w}}=2 \boldsymbol{w} \Sigma_{w}^{-1} \Sigma_{b}-2 \lambda \boldsymbol{w} & =0 \\
\rightarrow \boldsymbol{w} \Sigma_{w}^{-1} \Sigma_{b} & =\lambda \boldsymbol{w}
\end{aligned}
$$

Eq. (1.13) is satisfied for all tuples $(\boldsymbol{w}, \lambda)$ where $\boldsymbol{w} \in \mathcal{W}$ and $\lambda \in \mathbb{R}$ and $\mathcal{W}$ is the set of eigenvectors of $\Sigma_{w}^{-1} \Sigma_{b}$ and $\lambda$ the corresponding eigenvalues. The quantity of $\lambda$ is a measure of separation quality for its corresponding $\boldsymbol{w}$. Thus, by taking the eigenvectors sorted by their corresponding eigenvalues from largest to lowest, the projection directions of optimal class separability as measured by the Fisher criterion in the projected space are obtained. Note that in a $C$ class problem, $\Sigma_{b}$ is calculated from the sum of outer products of $C$ class mean vectors and thus its rank is at most $C-1$. Therefore, there exist only $C-1$ eigenvectors of $\Sigma_{w}^{-1} \Sigma_{b}$ with non-zero eigenvalues.

Plugging in the obtained result for $\boldsymbol{w}$ in the linear transformation (1.7), we obtain a discriminative function $g(\boldsymbol{x})$

$$
g(\boldsymbol{x})=W^{T} \boldsymbol{x}+w_{0}
$$

where $W$ contains the calculated eigenvectors as columns aggregated in a matrix and $w_{0}$ are the corresponding biases. A sample of an unknown class can now be classified by calculating $g(\boldsymbol{x})$ and attributing it to the class with the largest likelihood value. 
LDA becomes the optimal Bayesian classifier under two important assumptions, as will be shown in the following:

In a general formulation, given a certain measurement $\boldsymbol{x}$, we should classify $\boldsymbol{x}$ to any of the $C$ classes $i$ if

$$
P(i \mid \boldsymbol{x})>P(j \mid \boldsymbol{x}) \forall j \neq i, i, j \in\{1 \ldots C\}
$$

Read as: "Decide that $\boldsymbol{x}$ stems from class $i$ if the probability of class label $i$ is higher than that of any other class, i.e. class $i$ has the highest probability".

Applying the Bayesian rule between posterior and prior probabilities and plugging back into (1.15) delivers:

$$
\begin{aligned}
& P(i \mid \boldsymbol{x})=\frac{P(\boldsymbol{x} \mid i) P(i)}{\sum_{k} P(\boldsymbol{x} \mid k) P(k)} \\
& \frac{P(\boldsymbol{x} \mid i) P(i)}{\sum_{k} P(\boldsymbol{x} \mid k) P(k)}>\frac{P(\boldsymbol{x} \mid j) P(j)}{\sum_{k} P(\boldsymbol{x} \mid k) P(k)}
\end{aligned}
$$

Since the term $\sum_{k} P(\boldsymbol{x} \mid k) P(k)$ is positive and equal on both sides of the inequality, it can be eliminated, leaving:

$$
P(\boldsymbol{x} \mid i) P(i)>P(\boldsymbol{x} \mid j) P(j)
$$

There are two possible ways to obtain the class conditional probability density function, $P(\boldsymbol{x} \mid \cdot)$ : One way would be by estimation of the distribution, but this requires a great amount of measurements which is usually hard to obtain. Another way is to assume a probability distribution. Usually the following assumption is made:

Assumption 1: All measurements $\boldsymbol{x}_{k}$ stem from a multivariate Gaussian distribution, which is given by:

$$
P(\boldsymbol{x} \mid k)=\frac{1}{2 \pi^{\frac{k}{2}}\left|\Sigma_{k}\right|^{\frac{1}{2}}} \exp \left(-\frac{d_{m}}{2}\right)
$$

where $d_{m}$ is given as

$$
d_{m}=\left(\boldsymbol{x}-\boldsymbol{\mu}_{k}\right) \Sigma_{k}^{-1}\left(\boldsymbol{x}-\boldsymbol{\mu}_{k}\right)^{T}
$$


Therefore, (1.18) can be re-written as:

$$
\frac{P(i)}{2 \pi^{\frac{k}{2}}\left|\sum_{i}\right|^{\frac{1}{2}}} \exp \left(-\frac{d_{m}}{2}\right)>\frac{P(j)}{2 \pi^{\frac{k}{2}}\left|\Sigma_{j}\right|^{\frac{1}{2}}} \exp \left(-\frac{d_{m}}{2}\right)
$$

Eliminating $\frac{1}{2 \pi^{\frac{k}{2}}}$ on both sides and taking the natural logarithm leads to:

$\log P(i)-\frac{1}{2}\left|\Sigma_{i}\right|-\frac{1}{2}\left(\boldsymbol{x}-\boldsymbol{\mu}_{i}\right) \Sigma_{i}^{-1}\left(\boldsymbol{x}-\boldsymbol{\mu}_{i}\right)^{T}>\log P(j)-\frac{1}{2}\left|\Sigma_{j}\right|-\frac{1}{2}\left(\boldsymbol{x}-\boldsymbol{\mu}_{j}\right) \Sigma_{j}^{-1}\left(\boldsymbol{x}-\boldsymbol{\mu}_{j}\right)^{T}$

Equation 1.22 is referred to as quadratic discriminant analysis (QDA) and the separation lines between the classes are (hyper-)quadratics $\left(d_{m}\right.$ has been resubstituted in (1.22) to make the quadratic term apparent). It can readily be used as a classification rule, and the mean vectors and covariance matrices are approximated empirically using a set of training data.

This equation can only be simplified further under the following assumption:

Assumption 2: All classes $k$ share the same covariance matrix: $\Sigma_{i}=\Sigma_{j}=\Sigma$. Under this assumption, the term $-\frac{1}{2}|\Sigma|$ in 1.22 is the same on both sides and can be eliminated, leaving:

$$
\log P(i)-\frac{1}{2}\left(\boldsymbol{x}-\boldsymbol{\mu}_{i}\right) \Sigma^{-1}\left(\boldsymbol{x}-\boldsymbol{\mu}_{i}\right)^{T}>\log P(j)-\frac{1}{2}\left(\boldsymbol{x}-\boldsymbol{\mu}_{j}\right) \Sigma^{-1}\left(\boldsymbol{x}-\boldsymbol{\mu}_{j}\right)^{T}
$$

The term $\left(\boldsymbol{x}-\boldsymbol{\mu}_{k}\right) \Sigma^{-1}\left(\boldsymbol{x}-\boldsymbol{\mu}_{k}\right)^{T}$ can be split into: ${ }^{1}$

$$
\boldsymbol{x}^{T} \Sigma^{-1} \boldsymbol{x}-2 \boldsymbol{x}^{T} \Sigma^{-1} \boldsymbol{\mu}_{k}+\boldsymbol{\mu}_{k}^{T} \Sigma^{-1} \boldsymbol{\mu}_{k}
$$

with $\boldsymbol{x}^{T} \Sigma^{-1} \boldsymbol{x}$ being equal on both sides (assuming same covariance matrix for all

$$
\begin{aligned}
(\boldsymbol{x}-\boldsymbol{\mu})^{T} \Sigma^{-1}(\boldsymbol{x}-\boldsymbol{\mu}) & = \\
\left(\boldsymbol{x}^{T}-\boldsymbol{\mu}^{T}\right) \Sigma^{-1}(\boldsymbol{x}-\boldsymbol{\mu}) & = \\
\left(\boldsymbol{x}^{T}-\boldsymbol{\mu}^{T}\right)\left(\Sigma^{-1} \boldsymbol{x}-\Sigma^{-1} \boldsymbol{\mu}\right) & = \\
\boldsymbol{x}^{T} \Sigma^{-1} \boldsymbol{x}-\boldsymbol{x}^{T} \Sigma^{-1} \boldsymbol{\mu}-\boldsymbol{\mu}^{T} \Sigma^{-1} \boldsymbol{x}+\boldsymbol{\mu}^{T} \Sigma^{-1} \boldsymbol{\mu} &
\end{aligned}
$$

$\boldsymbol{\mu}^{T} \Sigma^{-1} \boldsymbol{x}$ is a scalar, thus: $\boldsymbol{\mu}^{T} \Sigma^{-1} \boldsymbol{x}=\left(\boldsymbol{\mu}^{T} \Sigma^{-1} \boldsymbol{x}\right)^{T}$. Further, $\left.(A B)^{T}\right)=B^{T} A^{T}$ and thus $(A B C)^{T}$ $=C^{T}(A B)^{T}=C^{T} B^{T} A^{T}$. This leads to: $\left(\boldsymbol{\mu}^{T} \Sigma^{-1} \boldsymbol{x}\right)^{T}=\boldsymbol{x}^{T}\left(\Sigma^{-1}\right)^{T} \boldsymbol{\mu}$. Since $\Sigma^{-1}$ is by definition a symmetric matrix, $\left(\Sigma^{-1}\right)^{T}=\Sigma^{-1}$. Therefore:

$$
\boldsymbol{x}^{T} \Sigma^{-1} \boldsymbol{x}-\boldsymbol{x}^{T} \Sigma^{-1} \boldsymbol{\mu}-\boldsymbol{\mu}^{T} \Sigma^{-1} \boldsymbol{x}+\boldsymbol{\mu}^{T} \Sigma^{-1} \boldsymbol{\mu}=\boldsymbol{x}^{T} \Sigma^{-1} \boldsymbol{x}-2 \boldsymbol{x}^{T} \Sigma^{-1} \boldsymbol{\mu}+\boldsymbol{\mu}^{T} \Sigma^{-1} \boldsymbol{\mu}
$$


classes!), and thus:

$$
\log P(i)-\frac{1}{2} \boldsymbol{\mu}_{i}^{T} \Sigma^{-1} \boldsymbol{\mu}_{i}+\boldsymbol{x}^{T} \Sigma^{-1} \boldsymbol{\mu}_{i}>\log P(j)-\frac{1}{2} \boldsymbol{\mu}_{j}^{T} \Sigma^{-1} \boldsymbol{\mu}_{j}+\boldsymbol{x}^{T} \Sigma^{-1} \boldsymbol{\mu}_{j}
$$

Thus for classifying an input vector $\boldsymbol{x}$, the function $g(\boldsymbol{x}, i)$ has to be evaluated for each class $i$ :

$$
g(\boldsymbol{x}, i)=\underbrace{\log P(i)-\frac{1}{2} \boldsymbol{\mu}_{i}^{T} \Sigma^{-1} \boldsymbol{\mu}_{i}}_{C g}+\boldsymbol{x}^{T} \underbrace{\Sigma^{-1} \boldsymbol{\mu}_{i}}_{W g}
$$

where $C g$ and $W g$ can be calculated readily during the training of the classifier. The classification rule is then simply to evaluate (1.31) for each of the classes and classify $\boldsymbol{x}$ to class $i$ if

$$
g(\boldsymbol{x}, i)>g(\boldsymbol{x}, j) \forall j \neq i, i, j \in\{1 \ldots C\}
$$

The value of $g(\boldsymbol{x}, i)$ is an indicator for the likelihood of the correctness of this classification. When the sum of all likelihoods for all classes is normalized to 1, each of the obtained values can be interpreted as a probability. Since in (1.22) the logarithm was taken for mathematical convenience, re-linearization of the likelihood values is advisable by exponentiation of each $g(\boldsymbol{x}, i)$ value, as proposed in [52].

\subsubsection{Regressors - It doesn't always have to be either-or}

In the previous section, the most classic machine learning methods applied to EMG signals for advanced myoelectric prosthetic control were introduced - classifiers. In pattern recognition, classifiers are used to assign one class label from a set of pretrained classes for a given input pattern. Therefore, they have hard boundaries and discrete outputs. In myoelectric pattern recognition, classifiers are usually used to determine the desired movement. In order to get an additional measure for the strength of that movement, the global amplitude of EMG signals (normalized to a maximum) is used and translated to the speed with which the selected movement is to be executed (proportional value). Other strategies have also been proposed [53]. In an entirely different approach however, one can directly estimate the movement speeds or forces for each involved degree of freedom (DOF). This is accomplished by performing a regression of the input features on the desired outputs. For example, in linear regression, a target vector $\boldsymbol{y}$ describing the instantaneous desired state of the 
prosthesis can be obtained by linear mapping of the input features $\boldsymbol{x}$ using a weight matrix $W$ :

$$
\boldsymbol{y}=W^{T} \boldsymbol{x}
$$

In a prosthesis with $2 \mathrm{DOF}, \boldsymbol{y}$ would be a two dimensional vector, $\boldsymbol{x}$ would be a $n$-dimensional feature vector and $W \in \mathbb{R}^{n \times 2}$. In order to obtain the fit that produces the least squared error between all measurements $X$ and corresponding given targets $Y, W$ could be obtained by simply multiplying (1.33) with $X^{-1}$ from the right:

$$
X^{-1} Y=W^{T}
$$

Evaluating (1.34) directly however is not possible in general, since $X$ is usually not symmetric and thus $X^{-1}$ not defined. Instead, the Moore-Penrose pseudoinverse [54] can be substituted:

$$
\left(X^{T} X\right)^{-1} X^{T} Y=W^{T}
$$

The resulting regression weights $W$ minimize the squared error between the produced estimates and the prompted targets. Linear regression is the most straightforward and simple regressor. It can be extended by regularization (ridge regression) and application of the kernel trick (kernel ridge regression) for non-linear estimations [55]. These regression methods require precisely labeled data, i.e., for each input vector the instantaneous target value has to be known. These can be acquired by tracking of the sound hand in mirrored bilateral movements $[42,43,46,55]$, the targets prompted to the subject or, in intact limb subjects, the produced grasping force $[48,56]$. However, also semi-unsupervised methods exist for this purpose, requiring only information on the active DOF but not the exact force trajectory. Such a method was proposed by Jiang et al. [45] and is inspired by the natural, synergistic ways in which muscles operate. Non-negative matrix factorization (NNMF) [57] was used to factorize a matrix of recorded muscle activations (= EMG envelopes) into a matrix of primitives and synergies. The inverse of the synergy matrix can later be used as the weight matrix as in (1.33).

In a slightly different type of approach, regression can also be performed by training an ANN to map the input features to some target prosthetic states or commands $[42,46,58]$. In this case, one network is trained for each DOF and the outputs 
are concatenated to yield the final movement commands for all DOF for the prosthesis.

Another type of regression that has been applied to EMG signals for myoelectric prosthesis control is support vector regression (SVR) [48,59], showing promising results. The main advantage of regression methods over classifiers is that they facilitate simultaneous estimation of several DOF. Therefore they allow for a close resemblance of movements to natural, sound arms and hands. Their common drawback however is that they can only cope with a limited number of DOF (typically 2), since including more results in very unstable estimation (as will be discussed further in Chapter 5). Furthermore, some of these techniques also require training data from combined movements together with their labels (ANN, SVR), which are time intensive to acquire in a sufficient amount. Therefore, methods which can extrapolate from single-DOF training data to multi-DOF movements during application such as linear regression, (kernel) ridge regression and NNMF are preferred [60].

\subsection{Current state of the art}

In commercial prostheses, none of the methods described above have been integrated so far in a clinically viable manner. However recently, a new controller called COAPT Complete Control $^{\mathrm{TM}}$ [61] has been presented, capable of sEMG pattern recognition for prosthetic control. The system emulates independent conventional electrode signals and can thus be used in conjunction with many commercial prosthesis controllers. It uses pattern recognition and allows the user to recalibrate anytime necessary. The commercialization of the product has just begun and while it still has to prove to prevail on the market, this is the first promising step towards commercial routine use of pattern recognition in upper limb prosthetic control.

From an academic point of view, the most important limitation of the current state of the art is that only very little studies have been conducted with amputees in a setup close to clinical practice. The online control of physical prostheses by subjects was rarely reported in literature. However, it has recently also been discussed that results from offline and online evaluations are only loosely correlated $[58,62,63]$. This underlines the importance of clinically relevant studies for a better estimation of the impact that newly developed methods have on the clinical outcome. The robustness of the investigated methods under such study settings has previously not been described. 


\subsection{Thesis goal and outline}

The goal of this thesis was to investigate sources of non-stationarity affecting the sEMG signals and to alleviate their effects on the machine learning based control of multifunctional myoelectric prostheses. This was to be done in clinically realistic study settings. The increased robustness of the developed estimation methods was to be shown in comparative offline as well as in online control investigations. Physical prostheses used by both able-bodied and amputee subjects were to be employed. The tests were designed to mimic real life scenarios in order to maximize the clinical relevance of the achieved results.

The further outline of this thesis is therefore structured as follows:

- In Chapter 2, a detailed review of non-stationarities, which affect the performance of EMG pattern recognition systems, is gathered from literature. A lack of knowledge on time influences (performance across days) is identified. Hence, in the further progress of this chapter, the development of a suitable evaluation paradigm for this influence is elaborated, measured, analyzed and discussed.

- In Chapter 3, a method capable of alleviating the detrimental effects of nonstationarities on sEMG pattern recognition is proposed. The development of the method is detailed and its applicability is demonstrated on test data acquired from able-bodied and amputee subjects. Specifically, the most influential nonstationarities identified in Chapter 2 are coped with. The accuracy obtained with this method is compared to that of the unprocessed classification stream as well as to results of other post-processing methods.

- In Chapter 4, a new multi-class proportional myocontrol algorithm for upper limb prosthesis control is proposed. It incorporates important insights obtained from the first two studies into a single, improved and novel control method. It is specifically developed for robust control of multifunctional prostheses under the influence of certain non-stationarities such as dynamic contractions. It inherently eliminates wrong movements of the prosthesis while providing direct proportional control values for smooth prosthesis control. The method is applied for the sequential control of a multi-DOF physical prosthesis in various test 
scenarios with able-bodied and amputee subjects and its superiority to two other, state of the art, control methods is demonstrated.

- In Chapter 5, the limitation of sequential control from the previous study is overcome by combining the introduced method with methods of simultaneous and proportional control across multiple DOF. This final system incorporates the knowledge gained from all previous evaluations and yields a robust, reliable and highly advanced control method for multi-DOF prostheses with simultaneous and sequential control for natural positioning of the wrist and robust, intuitive grasping. 


\section{$2 \quad$ Time related robustness}

The main shortcoming of current pattern recognition algorithms for sEMG for prosthetic control is believed to be a lack in robustness. Many sources of reliability reducing factors have been identified in previous studies. One of the most investigated factors in literature is the so called limb position effect $[42,64,65,66,67,68,69]$. This effect describes the influence of different arm positions on the recorded sEMG signals, such as lifting or stretching the arm. One possible reason for altered signals in elevated arm positions and thus decreased movement recognition is the activation of posture sustaining muscles such as the $m$. brachioradialis, responsible for lifting the forearm. Additionally, moving one's arm results in muscle displacement underneath the skin, causing the electrodes located on the skin surface to detect changed signals with respect to the neutral arm position. These effects are even more pronounced when additional weight is being sustained by the limb, e.g. when holding a heavy object or the end effector of the prosthesis itself [70], causing the socket to press against the stump. Recently, also the influence of arm motions while executing wrist and hand functions, such as lifting the arm or bringing the hand towards the mouth, has been investigated [68]. Unfortunately, no regularities of the described effects with respect to the limb positions and movements could be determined so far for automated compensation. Therefore, the only effective methodology proposed in literature for resolving the limb positioning effect so far is to heuristically include a variety of arm positions in the training set of the classifier $[64,65,66]$. In $[69,71,72]$ the utilization of an inertial sensor unit in the prosthesis was proposed. By including the orientation of the prosthesis in the feature set, a significantly increased robustness towards varying arm positions could be demonstrated. The common drawback of all the presented solutions however is that several arm positions have to be included in the data acquisition session for classifier training data collection. This can drastically increase the required training time and is fatiguing for the users. 
Other sources of non-stationarity that have already been identified and studied are the effects of electrode shifts $[40,73,74]$, dynamic contractions $[49,75]$, different contraction levels [76], muscle fatigue [77], impedance changes, movement strategy changes (mutual adaptation) [78], and psychological factors [79]. For coping with all of these factors, similar strategies as with the limb position effect have been proposed, i.e. including them in the classifier training set, with the same drawback as mentioned above.

One factor which has only received very limited amount of attention is time. How does a classifier trained with data of one day perform on the next day? And the day after, etc? Kaufmann et al. [80] conducted investigations in this direction, however in this conference publication only data of one healthy subject were included, allowing only for limited generalizability to a larger population and amputees. Therefore, in the study presented hereafter, the design and results of a study aiming to significantly extend our knowledge in this direction are presented.

The concept of this study, as well as the results have been published partially in similar form by me as first author in [81] and are extended here. Further, they have been used as a basis for the submitted paper [82]. Therefore, text or results reproduced from these manuscripts are not cited explicitly in the following.

\subsection{Methods and procedures}

In order to assess the stability over time of EMG pattern recognition, 7 able-bodied ( 5 male, 2 female, age $25.4 \pm 1.4$ years) and 4 male transradial amputee subjects with medium stump lengths were recruited to participate in this study. For all amputee subjects, the origin of amputation was traumatic, ranging from 1 to 21 years ago. Two of the amputees were right and two left hand amputees. Over the course of 5 days, each subject performed the same exercises each day. Prior to the start of the experiments, each subject was introduced to the study procedures and an informed consent was signed by the participants.

The subjects were seated comfortably in front of a computer monitor, leaning their back against the backrest of the seat. The upper arms were hanging in a relaxed position parallel to the torso, while the lower right arm was flexed in a $90^{\circ}$ angle, parallel to the floor, pointing forward. Eight double differential dry electrodes $(13 \mathrm{E} 200=50 \mathrm{AC}$, 


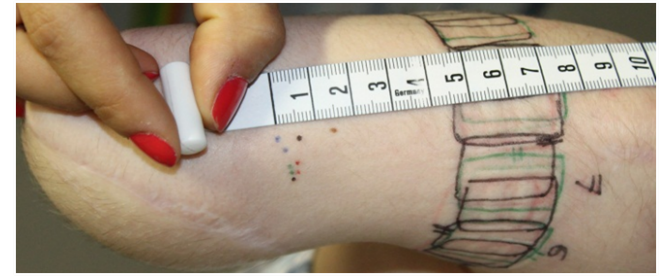

(a) Stump Amputee1

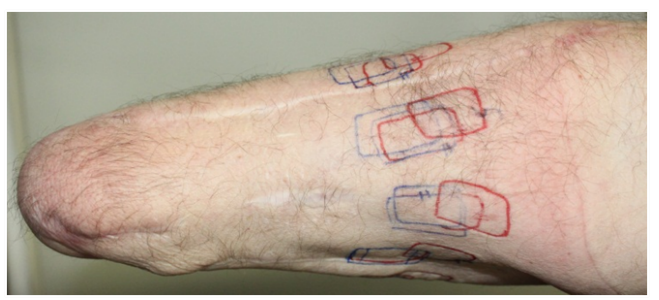

(c) Stump Amputee3

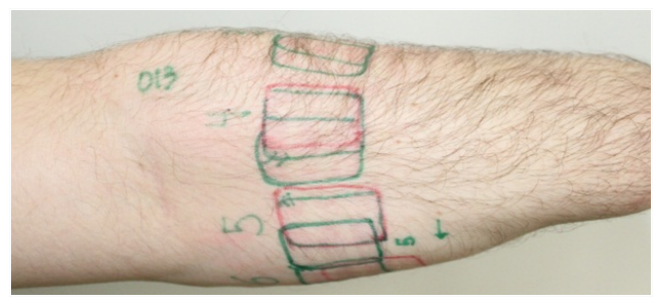

(b) Stump Amputee2

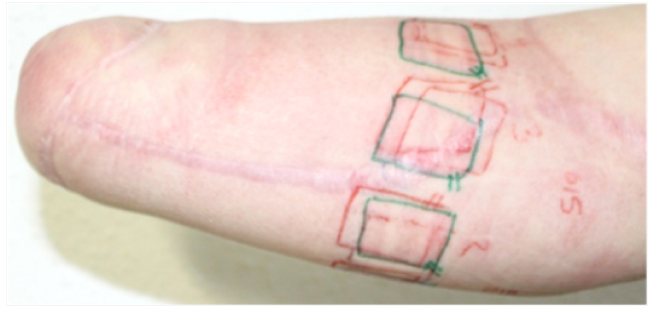

(d) Stump Amputee4

Figure 2.1: Stumps of the subjects, with the electrode locations marked after doffing to quantify the electrode displacements.

Otto Bock HealthCare Products GmbH, Vienna, Austria) were placed equidistantly around the circumference of the right forearm in able-bodied subjects and on the stump of the amputees, approximately $7 \mathrm{~cm}$ distal to the elbow. All able-bodied subjects were dominant right handed. In case of dry skin, the electrode placement locations were cleaned and moistened, since this reduced the time required for electrodeskin contact to settle. The necessity of this measure was judged by the obtained signal quality.

For each amputee, a custom prosthetic socket housing the 8 sEMG electrodes was manufactured by an orthopedic technician. This allowed for a very realistic test setup in which doffing/donning effects could be observed like with an actual, personalized prosthesis of the wearer in a clinical routine use.

The recorded signals were filtered and amplified by the active electrodes $(20-450 \mathrm{~Hz}$, $50 \mathrm{~Hz}$ notch filter, output 0-4.5 V). The such conditioned raw signals were sampled at $1 \mathrm{kHz}$ by the Axon Bus ${ }^{\circledR}$ master (Otto Bock HealthCare Products GmbH, Vienna, Austria) with 10 bit resolution and wireless transmitted via Bluetooth to a personal computer, where the data were recorded and saved using a custom application. Before the start of the experiment, the exact position of each electrode on the skin was marked using a skin friendly, water and sweat resistant marker and renewed daily as needed. This way, the electrodes could be placed on the same locations each day. 


\subsubsection{Test protocol}

Following the above described preparations, each subject was introduced to the correct performance of the following 8 movements: wrist supination (WS), wrist pronation (WP), wrist flexion (WF), wrist extension (WE), hand opening (HO), key grip $(\mathrm{KG})$, fine pinch (FP), and no movement (NM). The instructions given to the ablebodied subjects were to perform each movement as consistently as possible in each repetition and to focus on exclusively performing only the prompted move. For example, during WE some subjects tended to also spread their fingers (= opening of the hand), which they were then corrected to only extend the wrist joint while keeping their fingers relaxed. This was done for every movement. With amputee subjects, their phantom limb movements were explored until consistent and distinguishable movements were found by visual inspection of the sEMG signals.

For each of the 8 movements, the maximum long term voluntary contraction (MLVC), defined as the maximum contraction that the subject was able to hold over a period of approximately $20 \mathrm{~s}$, was determined. This maximum was later used to scale the prompted movements. For data collection, the subjects received biofeedback on their current total exerted force by calculating the sum of RMS values of all electrodes, scaled to the MLVC level. A red cursor was displayed on the computer screen, whose $\mathrm{y}$-coordinate was proportional to the exerted force and which propagated along the $\mathrm{x}$-axis with time.

During one run, subjects were asked to track trapezoidal shaped profiles $\left(t_{\text {rise }}=1 \mathrm{~s}\right.$, $t_{\text {plateau }}=3 s, t_{\text {fall }}=1 \mathrm{~s}$ ) with plateau heights of $30 \%, 60 \%$ and $90 \%$ of the MLVC. Hence, one run consisted of $8 \times 3=24$ movements. The movement which was to be performed for each profile tracking was indicated to the subject with audio and visual cues. In total, each subject completed 5 runs in one session. Able-bodied subjects completed 3 sessions per day and amputees 2 sessions. Between sessions, the electrodes were removed for approximately 15 minutes to study the effect of electrode doffing-donning. In able-bodied subjects, the electrodes were placed again on the exact same locations as marked on the skin before doffing. In amputees, natural doffing-donning shifts occurred and were quantified by measuring the electrode displacements between sessions and days. This test protocol was repeated over five consecutive days. Subjects were granted sufficient breaks between each run to avoid fatigue. 


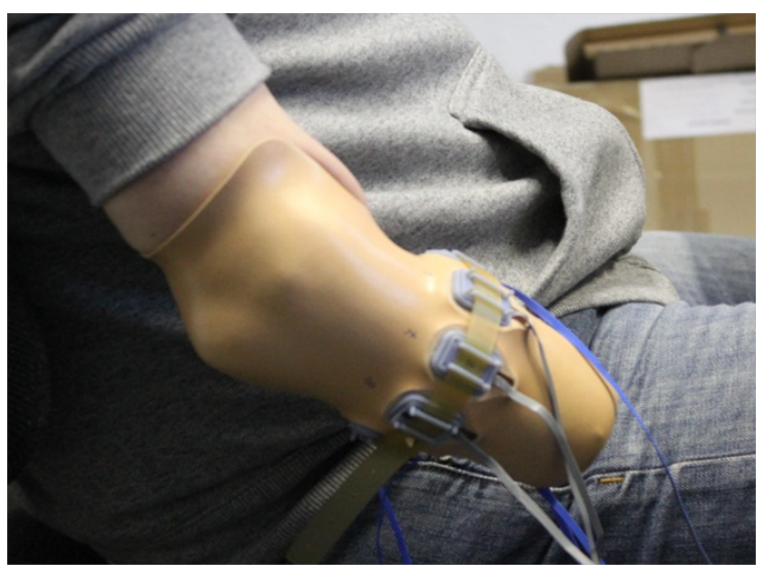

Figure 2.2: The test socket manufactured for Amp4 for this study. A similar socket housing the 8 electrodes was manufactured for each amputee to participate in this study.

\subsubsection{Signal processing}

The four time domain features RMS, ZC, SSC and WL as introduced in Section 1.4 were calculated from the signals, which were split in windows of $128 \mathrm{~ms}$ length with $78 \mathrm{~ms}$ overlap. Only the static parts of the contractions (3s plateau center of each movement) were considered for this study. All classification evaluations were performed offline using the LDA classifier as introduced in Section 1.5.1. A separate classifier was trained with data of each day of recordings and the data of all days were tested with each classifier. All data of one day were used for classifier training and all data of each test day were classified. For within-day evaluation, a five-fold crossvalidation was performed, i.e. $4 / 5^{\text {th }}$ of data from that day were used for training and $1 / 5^{\text {th }}$ for testing, permuted until all data were used for testing once. The classification accuracy (ratio of correct classifications and total classifications) is reported and is shown as mean \pm standard deviation, calculated across subjects.

\subsection{Results}

All subjects were able to complete the full study. Exemplary sEMG signals recorded from one amputee and one able-bodied subject are shown in Figure 2.3

In the setup of this study, a time difference of 1 to 4 days between classifier training set and testing set could be investigated as shown in Table 2.1. 


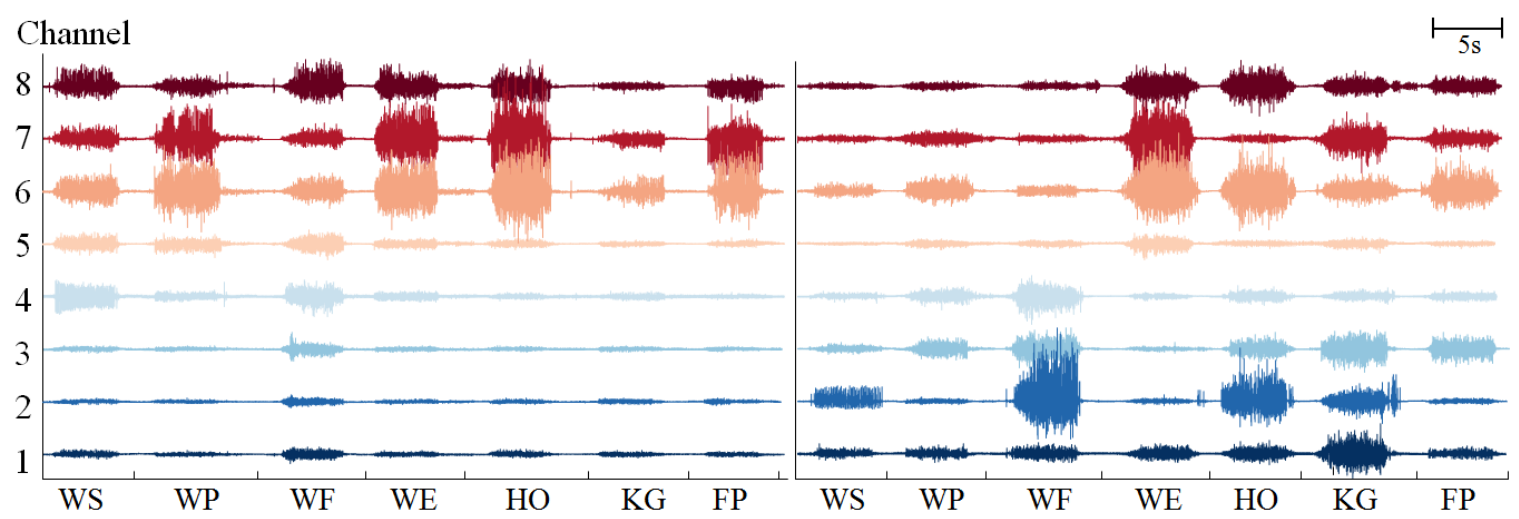

Figure 2.3: Exemplary sEMG signals for all 7 active movement classes investigated in this study of a representative amputee (left) and able-bodied subject (right). By visual inspection, the sEMG patterns appear well distinguishable per class for the able-bodied subject, however in the amputee subject, the patterns only differ slightly between most of the movements

Table 2.1: For comparing the robustness across days, a total of 4 inter-day differences could be evaluated.

\begin{tabular}{cccc}
1 Day & 2 Days & 3 Days & 4 Days \\
\hline Day1 $\leftrightarrow$ Day2 & Day1 $\leftrightarrow$ Day3 & Day1 $\leftrightarrow$ Day4 & Day1 $\leftrightarrow$ Day5 \\
Day2 $\leftrightarrow$ Day3 & Day2 $\leftrightarrow$ Day4 & Day2 $\leftrightarrow$ Day5 & \\
Day3 $\leftrightarrow$ Day4 & Day3 $\leftrightarrow$ Day5 & & \\
Day4 $\leftrightarrow$ Day5 & & &
\end{tabular}

The average classification accuracy within each day was $97.6 \pm 1.4 \%$ for able-bodied subjects. For amputees, the individual within day performances were 96.2\%, 75.3\%, $67.3 \%$ and $79.3 \%$. From these peak performances, substantial decreases in accuracy were found when the test data where drawn from days different than the day on which the classifier was trained. The average drop in accuracy from one day to the next was $8.3 \%$ in able-bodied subjects and $6.8 \%$ in amputees. With an increasing number of days between the training and testing data set, the recognition error increased monotonically up to $13.6 \%$ and $19.1 \%$ with the maximum investigated of 4 days distance ( $c f$. Table 2.1) in able-bodied and amputee subjects, respectively. The average performances of all combinations of training and testing days are summarized in Figure 2.4 and the average decrease of classification accuracy as function of days between training and testing is summarized in Figure 2.5.

In a further analysis it was investigated whether a certain subgroup of movements caused a majority of the mis-classifications. It was found that HO, FP and WS were 


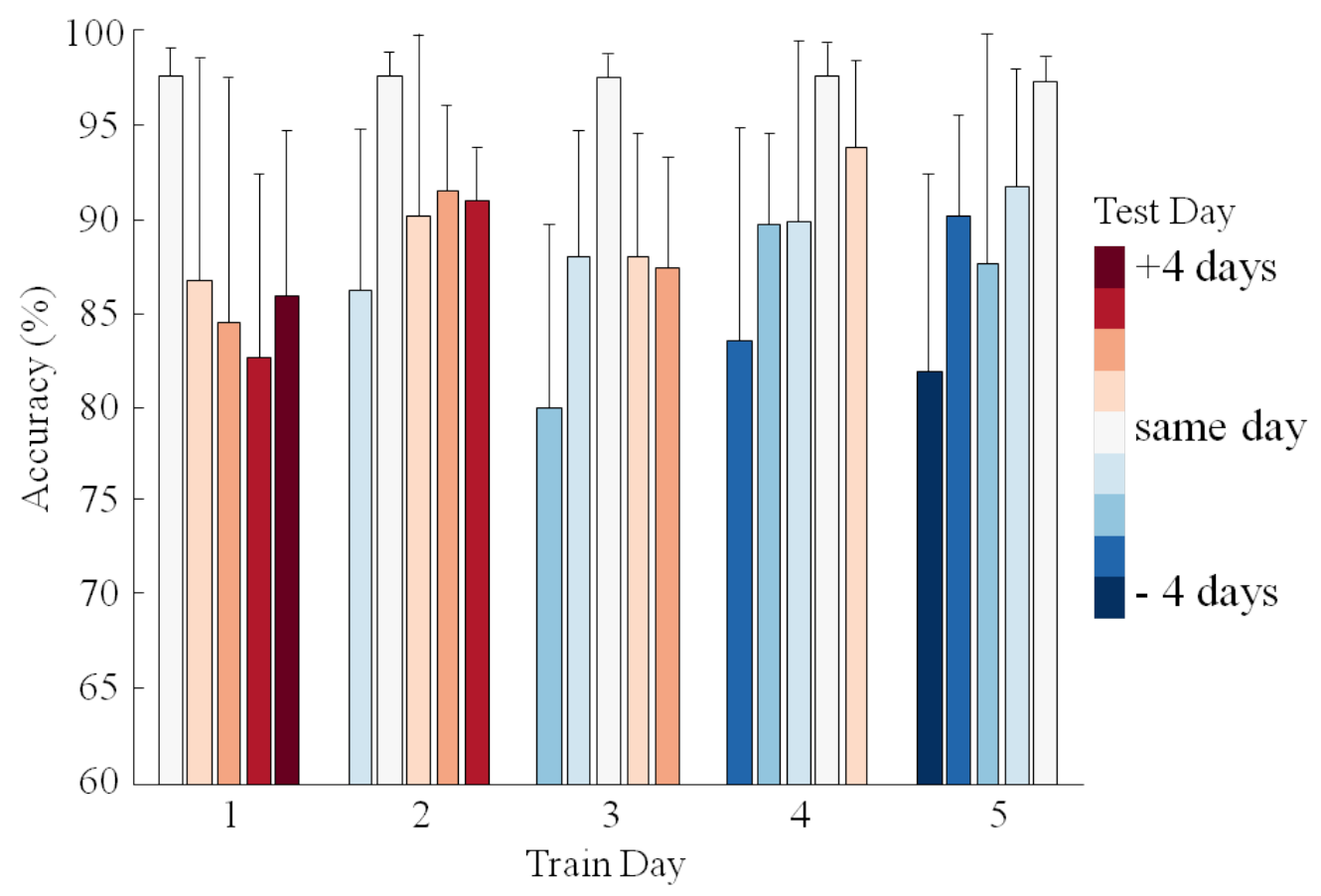

(a) Able-bodied subjects

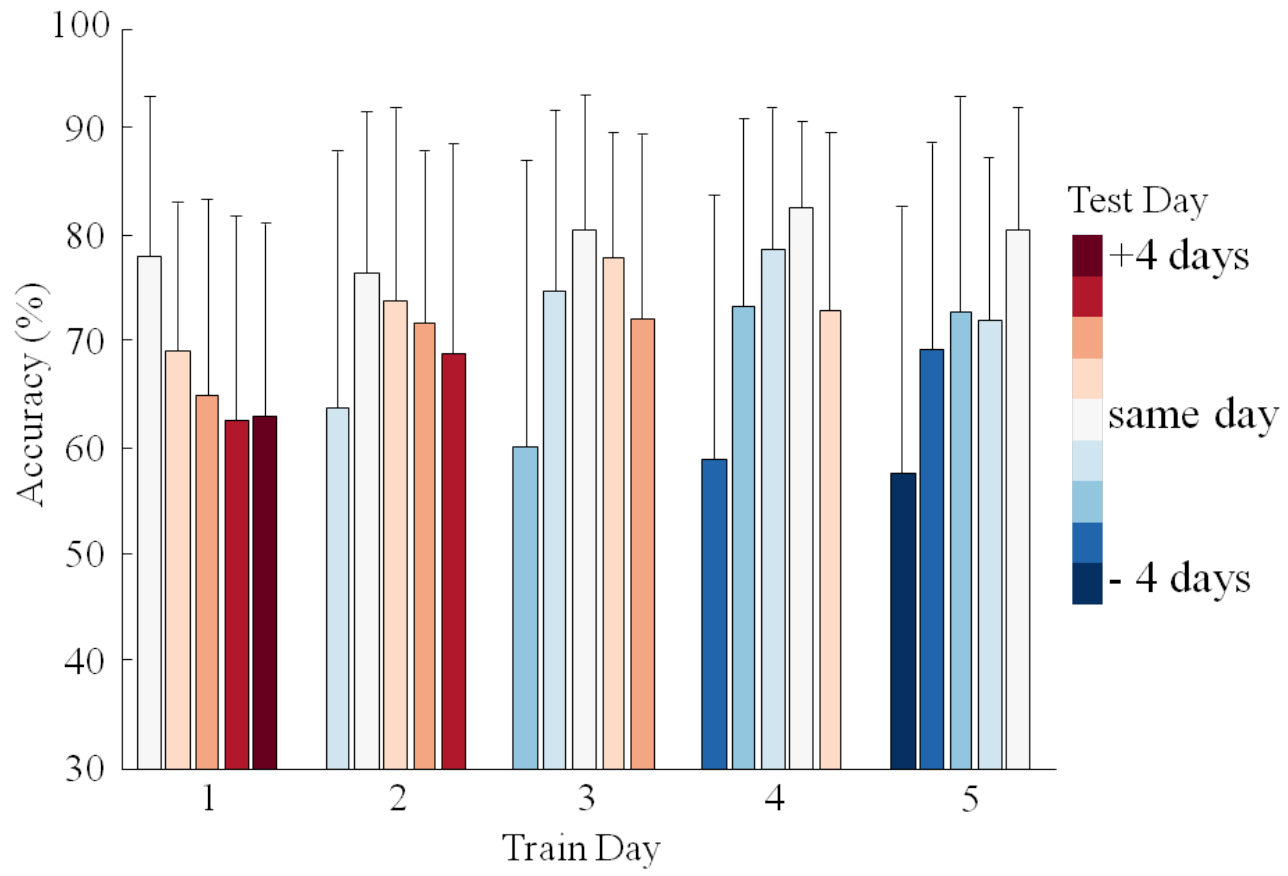

(b) Amputee subjects

Figure 2.4: Results of classification accuracy over time for (a) able-bodied and (b) amputee subjects. Reused and modified with permission [81] (C2013 IEEE. 


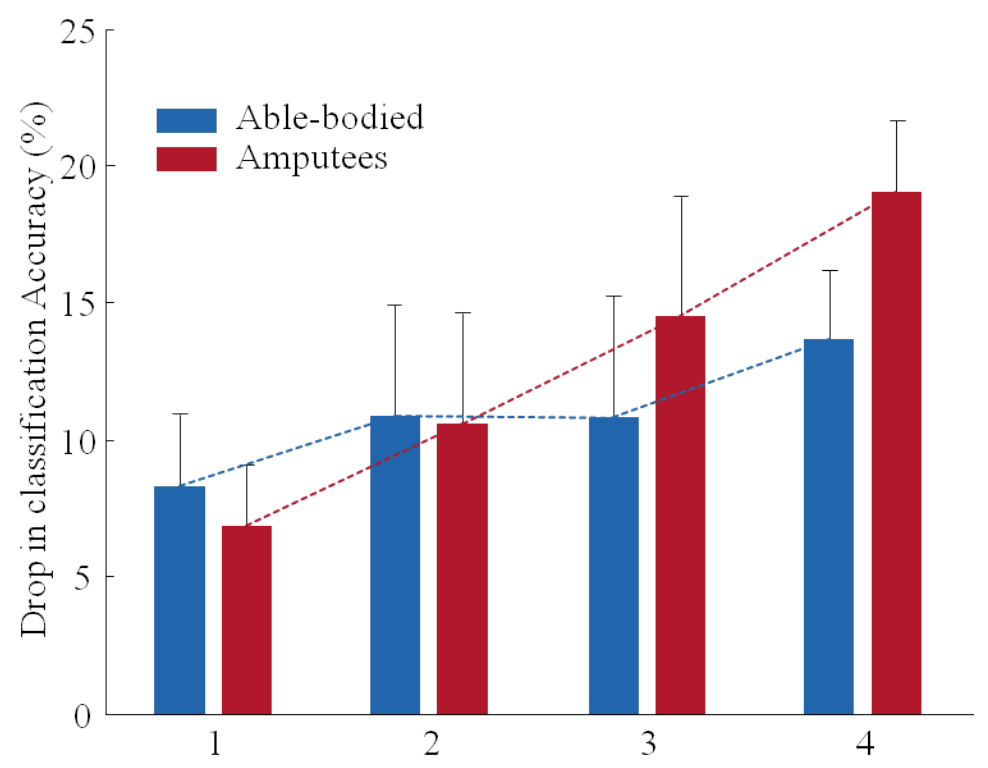

Days between training and test data set

Figure 2.5: Average drop of classification accuracy as a function of days between training and testing day compared to within-day testing for able-bodied and amputee subjects.

the movements which were involved in most of the classification errors. Together, these 3 movements accounted for $52 \%$ of all mis-classifications (Figure 2.6(b)).

As described in the introduction of this chapter, one non-stationarity whose influence on classification accuracy has often been investigated, was the shift of electrodes. However, in none of these studies a quantification of electrode displacements between two donnings has been reported. Instead, in many studies a rough estimate of usually several millimeters up to a few centimeters was assumed. In this study, due to the realistic setup with an individually manufactured prosthetic socket for each of the four amputees and its longitudinal character, the present investigations offered a good basis for quantifying the electrode displacements after donnings between the two sessions per day and also on consecutive days. Therefore, representative results for a real use case in amputees were accessible.

The average electrode displacements in longitudinal direction (along the forearm) were around $6 \mathrm{~mm}$ or less both across sessions and days $(3.9 \mathrm{~mm}$ on average across all sessions and $5.5 \mathrm{~mm}$ across days). In the perpendicular direction, shifts were less than $9 \mathrm{~mm}$ across sessions and less than $6 \mathrm{~mm}$ across days $(5.9 \mathrm{~mm}$ on average across all sessions and $5 \mathrm{~mm}$ across days). 


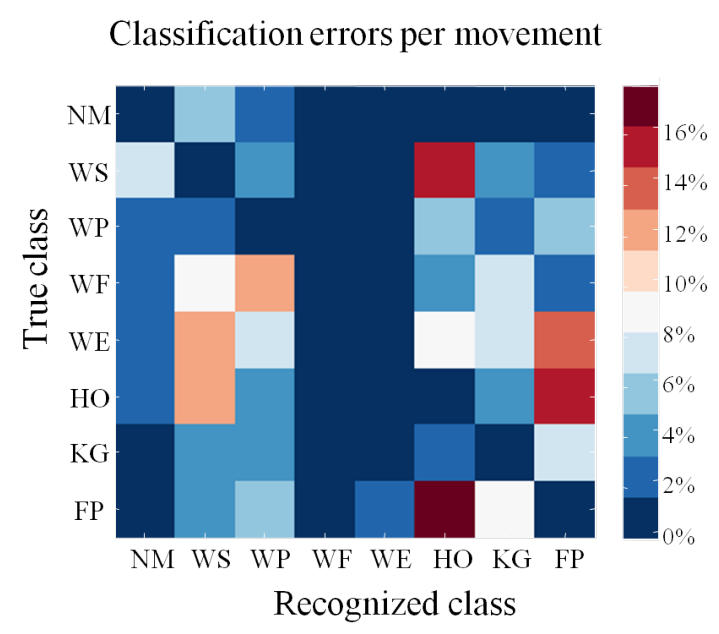

(a)

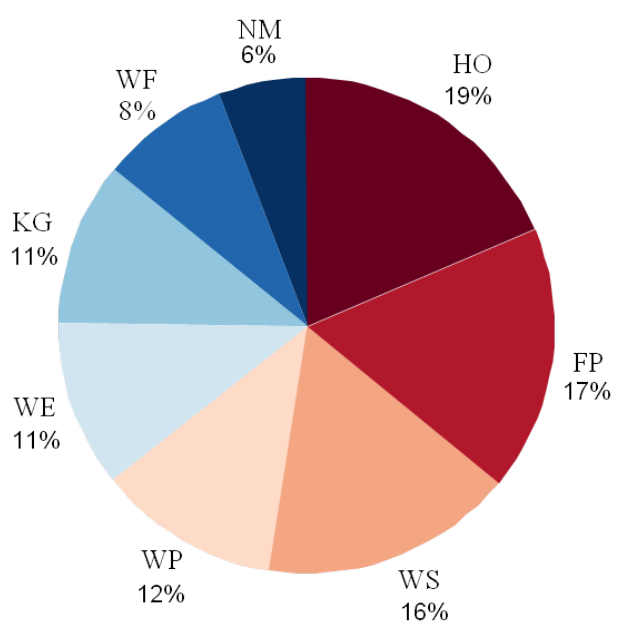

(b)

Figure 2.6: Average confusion matrix of mis-classifications, scaled to minimum and maximum (a) and the percentage of total mis-classifications each movement accounted for (b). It can be seen that WS, WP, HO and FP were the most difficult classes for classification. NM and WF were the least difficult ones.

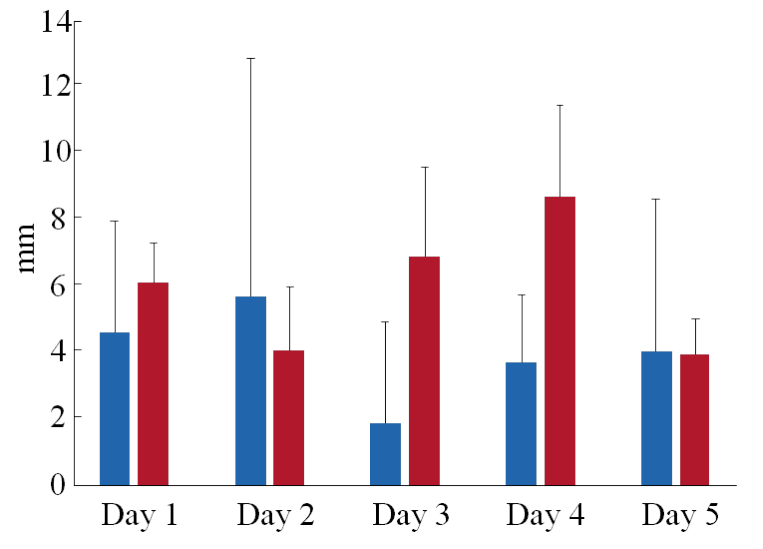

(a)

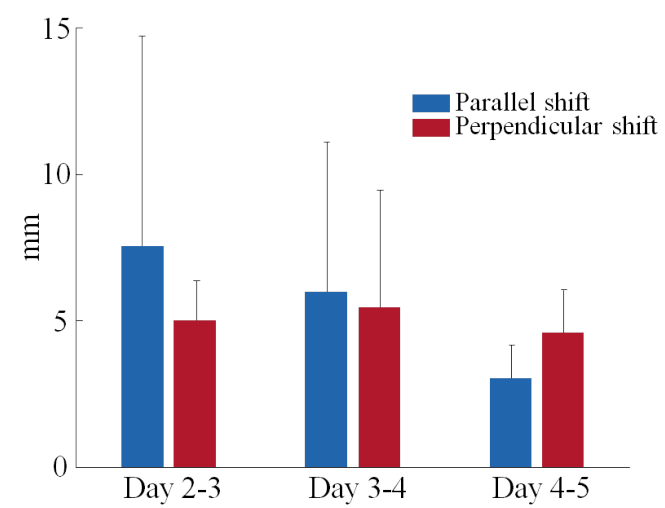

(b)

Figure 2.7: Quantified electrode shifts in amputees with their custom made sockets (a) between the two sessions of one day and (b) between the consecutive days. The shifts between the first and second day were not measured. 


\subsection{Study discussion and conclusion}

In the presented study, a research question which had only received minor attention previously was addressed. Many non-stationarities negatively affecting the sEMG signals for pattern recognition have been described in literature, but the importance of time related effects was unknown. Therefore, in this study a total of 11 subjects (including 4 amputees) were recruited to perform a set of 8 wrist and hand movements while their sEMG signals were recorded from the forearm. The recording sessions were repeated over the course of 5 consecutive days. In an offline analysis, the signals recorded from each day were used to train a classifier and the signals of all days were tested with this classifier. The maximum classification accuracies were obtained when the training and testing data set were acquired on the same day (using a 5 -fold cross-validation). In able-bodied subjects this accuracy was close to $100 \%$, which is in good correspondence with other studies achieving comparable results in similar study settings [28]. In amputees, the results of the within day accuracy were much lower with an average of $79.5 \%$, however reporting the average is deceiving in this case, since the results were quite different among subjects. This was likely the case because Amp1 had extensive experience with pattern recognition training prior to participating in this experiment. This shows that subject training is likely an important factor, which was also reported in [83], especially in amputees. In able-bodied subjects, proprioception and visual feedback of the moving hand made it easier to perform consistent, repeatable movements.

The most important finding of this study was that the further training and test days were apart, the more the classification accuracy decreased. This decrease was monotonic and did not reach a plateau after the 5 days investigated in this study. It is thus possible that after longer time periods a further decrease in accuracy has to be expected. While the investigation of 5 subsequent days is considerably longer than in the majority of all other studies, which are only conducted in one session, this is still a limitation of the presented study which has to be addressed in future investigations. Another question that merits particular attention in a dedicated study is towards the origin of the observed time dependent degradation. This investigation was outside the descriptive scope of this study, but has been started by Paredes, Amsüss et al. [82]. In this yet unpublished draft, inspired by the contribution of Bunderson et al. [83], several measures were assessed in feature space to quantify the origin of mis- 
classifications with the data set acquired in the here presented study. Furthermore, in [84] (see Chapter 3) and [85], strategies for alleviating the observed day-to-day degradations with this particular data set were proposed.

A further limitation of the analyses presented here was the restriction to offline investigations. It is possible that some of the observed classification degradations over time could be corrected by the user during online control by slightly adapting the way movements are performed. However, this implies that relevant feedback for success of the changed movement strategies must be given to the user, otherwise he is left to search blindly and without guarantee of improvement. Such a feedback is not yet available. Although it is possible to visualize the data by projecting them into a 2 or 3-dimensional space using e.g. principal component analysis (PCA) [86], a direct and easy to understand guide for constructive adaptation of user behavior has not yet been proposed. In this study, the occurrence of data shifts across time and their detrimental effects on sEMG classification have been demonstrated. The capabilities of algorithms and users to adapt to these changes however have to be shown in future studies.

As an additional result of this descriptive study, the electrode shifts which have to be expected after doffing and donning across sessions of the same day and different days were quantified with the individually manufactured prosthetic sockets used for amputees in this study. As a result, it was demonstrated that shifts usually occur below $1 \mathrm{~cm}$. It has to be pointed out however that these results may vary with the quality of the socket fit and with the condition of the residual limb, c.f. Figure 2.1(c). The numbers reported here should serve as a guideline for future investigations regarding the influence of electrode shifts on sEMG pattern recognition systems. 



\section{Self correcting classification system}

In the previous chapter, several non-stationarities, which are known to affect the classification accuracy of EMG pattern recognition based myoelectric control, were partially discussed from literature and in particular the influence of time related effects was investigated in a dedicated study. Previous to this work, extensive knowledge on multi-day testing had not been published. In the present study, the data which were acquired during the experiments of the first study were further analyzed. A methodology to counteract the decreases in classification accuracy observed in dayto-day testing and in presence of other non-stationarities is being elaborated.

The objective of this study was to design and validate a method capable of detecting and eliminating its own mistakes, based on the stream of observations made in the past. In fact, most pattern recognition algorithms proposed in the past regarded each feature vector as an independent observation that was to be classified. For example, in an 8 class problem, with conventional classification methods it was possible to classify 8 consecutive samples all to different classes. Since in myoelectric control new decisions are usually made approximately every $50 \mathrm{~ms}$, this means the system would allow the user to switch to all movements within less than $500 \mathrm{~ms}$, which is physiologically not meaningful. In a much more realistic scenario, for example given a stream of 10 samples which were classified to the same movement, it is very likely that the $11^{\text {th }}$ sample will also belong to this class. Hence, if the classifier would suggest a new label for this sample, this decision should be questioned and accepted only in case of high probability for correctness of this prediction. Similar behavior could be achieved by a simple low-pass or moving average filter, however this would inevitably introduce delays in the control. An illustration of this idea which is fundamental for the following is given in Figure 3.1. 


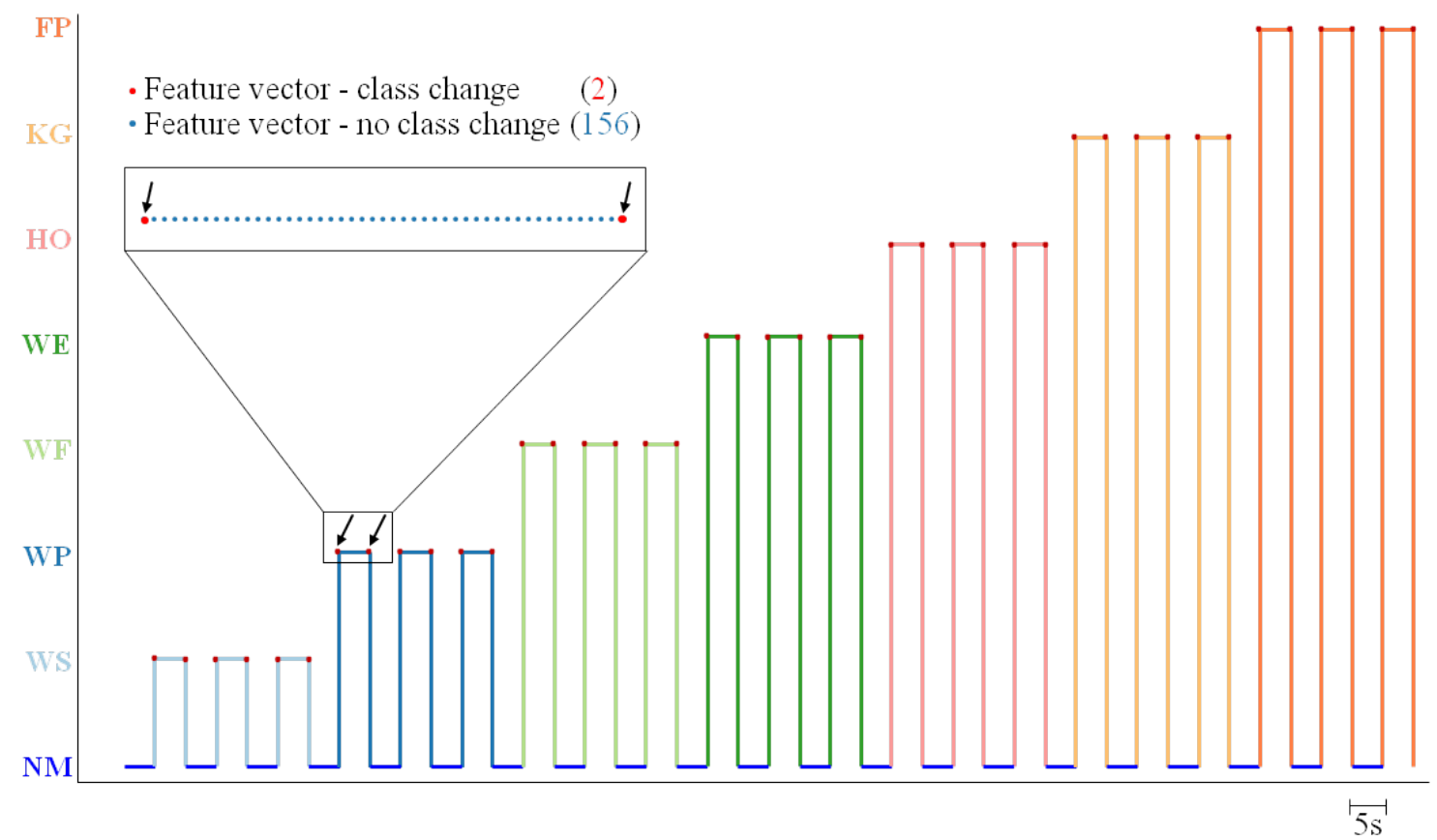

Figure 3.1: Schematic representation of performing 7 different movements with 3 repetitions each ( 1 run). Contractions are sustained for a period of 5 seconds. Class switches between two adjacent estimates are far less likely than staying in the previous class. In the shown example, classes changed at red points. In a recording of 22 minutes (5 runs), 23978 feature samples were acquired. In 23768 out of these, the movements of the subject did not change and only 210 times there was an actual switch between movements, representing a total of $0.87 \%$ of all estimates. This a priori information should be considered during classification. The same rational holds for less ordered movements than shown here for illustration during real life application. 
Therefore, in this study a method was proposed to compute such a probability index, which allows for overriding decisions with low likelihood of correctness. The derived method was tested on an extensive set of data. Its effectiveness was compared to the unprocessed classifier predictions as well as to 3 other post-processing methods.

The concept of this study, as well as the results have been published in similar form by me as first author in [84]. Therefore, text or results reproduced from this manuscript are not cited explicitly in the following. All figures and tables were reproduced with permission.

\subsection{Methods and procedures}

For this study, the data which were presented in Chapter 2 were taken as a basis for the development and evaluation of the proposed method (data of 7 able-bodied and 4 amputee subjects). As introduced in this previous study, several non-stationarities are known to affect the sEMG signals, resulting in degradation of classification accuracy if not compensated. From these inconsistencies, the following were included in the present data set:

1. The onset, static phase, and relaxation phase of the contraction were included in the data set, so that both static and dynamic phases were considered. In [49] the difficulty of classifying transient movement phases has been demonstrated.

2. Weak, medium and strong contractions corresponding to $30 \%, 60 \%$ and $90 \%$ MLVC were considered, along with the transitions in between. This is in contrast to other studies, where only one, user chosen, contraction level was investigated $[87,88]$.

3. The training and testing sets were recorded with one day difference: a classifier was trained with data of a particular day and data from the subsequent day were tested. This was repeated with a total of 5 days (first column in Table 2.1). Inevitably, a variety of non-stationarities were thus included simultaneously:

(a) Electrode shifts

(b) Impedance changes

(c) Socket fit (amputees)

(d) Psychometric factors such as subject motivation and concentration 
For reference, also the data within each session were classified in a 5-fold crossvalidation. This represented a scenario where these influences were minimized, which allows for an estimation of the effects of these non-stationarities.

4. Amputees were included in the study which are known to have more difficulties in producing consistent and separable movement patterns (e.g. see Chapter 2)

In addition to these mentioned non-stationarities, the no movement gesture was not actively classified. This was motivated by the fact that in preliminary evaluations it was observed that this movement was easy to be classified correctly, since compared to the active movement classes, the changes of this class were negligible. Thus, for this movement consistently more than $95 \%$ recognition accuracy were obtained, regardless of the method investigated. It was therefore not considered for the analysis in order to avoid a bias in the results. However, mis-classifications of active classes to the rest gesture were naturally taken into account.

In the following, the development and evaluation of a method is presented, capable of alleviating the combined effect of all of the above mentioned non-stationionarities known to degrade the classification accuracy .

\subsubsection{Self correction system}

The concept of the self correcting system was based on observations that were made during the conduction and evaluation of the study described in Chapter 2. The following insights were gained:

- Mis-classifications usually occurred during the onset and relaxation phase of movements.

- The likelihood output of the classifier was found to correlate with the correctness of the classification.

- The time history of classification stream contained information on the reliability of a classification (i.e. frequent classification output changes were correlated with mis-classifications).

- The contraction level was found to have importance - low strength contractions were usually more difficult to classify correctly than stronger contractions. 
Based on these observations, a methodology for eliminating mis-classifications was derived.

In a first attempt, a variety of features to reflect the observations described above quantitatively were empirically extracted from the classification stream, as summarized in the following list:

- Absolute value of EMG RMS

- Variance (change) of the EMG RMS value

- Classification likelihood output

- Number of subsequent consistent raw classification results

- Number of changes in output class

- Last accepted decision is among top 3 of classifier output

- 1-vs-rest classifier output of the last accepted class

- Detect onset/offset of move

Out of this list, the RMS value and classifier likelihood were identified to contain the most relevant information. Furthermore, the time history of these values was identified to be of great importance (e.g. a significant change in the RMS value indicated a dynamic contraction). Therefore, it was decided to use the history of 10 samples of the EMG RMS value and classifier likelihoods as final indicators for classifier confidence. A multilayer perceptron ANN (MLP-ANN) was chosen to automatically obtain and optimize a mapping function from these inputs to the desired confidence measure at its output stage. For this purpose, a number of samples had to be extracted from the training data, which were used to teach the ANN examples of trustworthy and non-trustworthy classifier outputs.

The details of the implemented system are described in the following section.

\subsubsection{Implementation of self-correction mechanism}

As for the first study, the LDA classifier was chosen as the base classifier for the system. For the purpose of identifying mis-classifications, a 3 layer MLP-ANN was implemented. The MLP-ANN had 22 input nodes (the current and past 10 EMG RMS values and maximum classifier likelihood). In a feed-forward structure, these inputs were weighted and forwarded to a hidden layer comprised of 8 neurons. The 
final output layer had only one neuron which produced continuous output in the interval $[-1 ;+1]$, where +1 was interpreted as high confidence in the classifier output and -1 as the opposite. All layers were connected with linear transfer functions and the output was limited to the interval $[-1 ;+1]$ by a hyperbolic tangent sigmoidal transfer function. For training of the MLP-ANN weights, the Levenberg-Marquart (LM) backpropagation algorithm was used. For the entire implementation of the MLP-ANN the Matlab ${ }^{\circledR}$ Artificial Neural Network tool box was used. The data set which was used for training the LDA classifier was also used for training of the MLP-ANN, therefore no additional training data to those needed for training the conventional system were needed. All data were selected to train the LDA. Subsequently, the same data were applied to the obtained classifier. For each data sample, a +1 was assigned in case of correct recognition and a -1 in case of erroneous output. Then, $80 \%$ ( 4 of the 5 runs) of the data were applied to train the ANN network with the inputs as described above and the targets for each sample as either +1 or -1 , depending on whether the LDA had classified it correctly or not. The remaining $20 \%$ of the training samples were used as a validation set for the MLP-ANN. Since the LM optimization algorithm does not guarantee convergence to a global optimum, a total of 5 networks with different randomly initialized weights was trained. The one with the lowest error rate on the validation set was used in all further analyses.

In the application phase of the system, a new feature vector was first classified by the LDA classifier, then the ANN output was evaluated. The described system is depicted schematically in Figure 3.2.

A trust index at time $t(T I(t))$ was computed from the raw network output $n(t)$ as follows:

$$
T I(t)=|T I(t-1)|^{(\alpha \cdot n(t))}+\beta(t)
$$

where $\alpha$ is a filter constant and $\beta(t)$ is given by

$$
\beta(t)= \begin{cases}\beta(t-1)+\frac{1}{200} & \text { if LDA class output consistent } \\ 0 & \text { if } T I(t-1)-T I(t-2)>0.5 \\ \beta(t-1) & \text { otherwise }\end{cases}
$$

The constant integration factor of $\frac{1}{200}$ was selected to reflect the increase of confidence with consistent LDA predictions at a maximum rate of 0.1 per second. In preliminary 


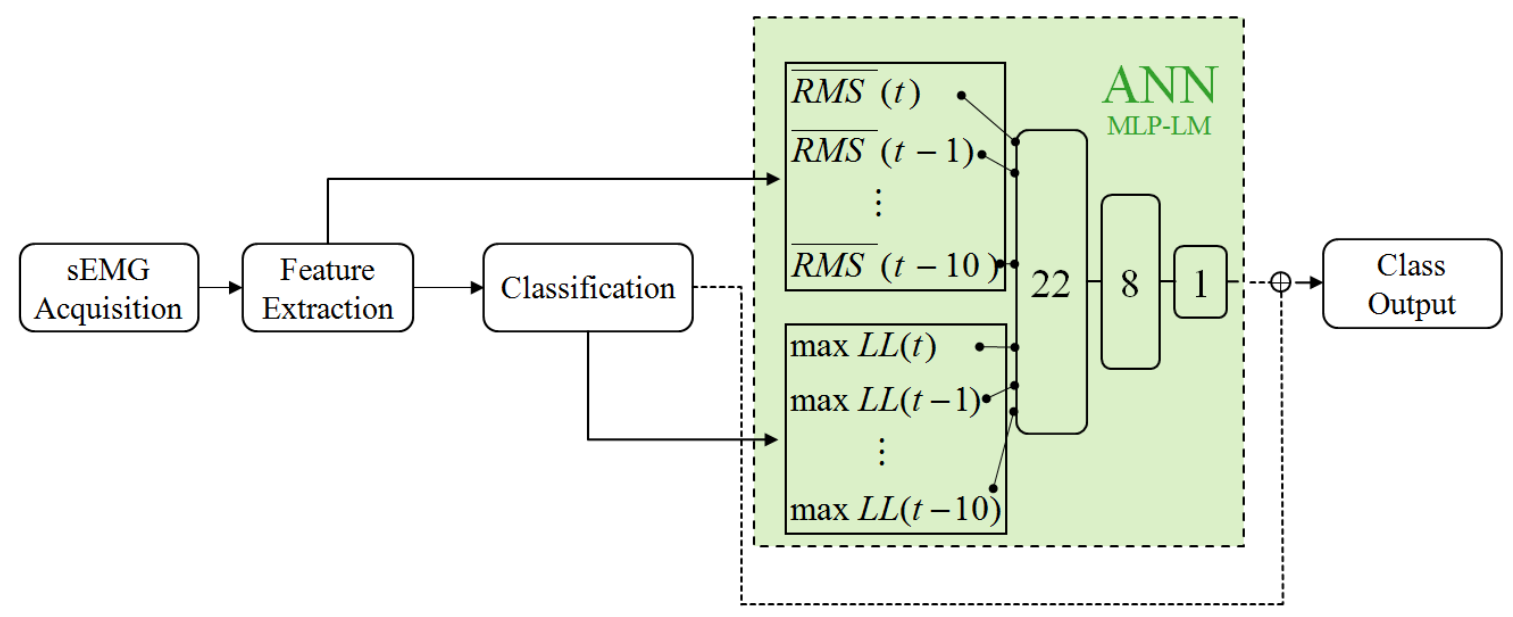

Figure 3.2: Schematic representation of the self-correcting classification approach. After classification using LDA, the MLP-ANN output is evaluated. The two results are then merged (correction of raw classification using ANN output) to a final decision of the system. Reused and modified with permission [84] @2014 IEEE.

investigations the exact value of this integration constant was found to be uncritical for values $>0$, hence this value was chosen without further optimization.

In (3.1), TI, and $\beta$ were initialized to 0.5 and 0 respectively. The smoothing factor $\alpha$ was varied in 9 steps between 0.1 and 0.9 . In case of a $T I(t)$ value above a certain threshold, the classifier decision was not altered. In case of a low confidence output however, the classification decision was dismissed and replaced by the previously accepted class. The threshold $\theta$ was varied in 100 steps from 0 to 1 . As a result, a 2-dimensional grid search for optimizing $\alpha$ and $\theta$ was performed to find the pseudooptimal values. Two different variations of this optimization were considered and termed as follows:

1. ANN-IND: The parameters $\alpha$ and $\theta$ were optimized for each subject and day individually. This optimization was expected to yield the best results.

2. ANN-GO: In order to find a generalizable solution that does not require individual optimization, $\alpha$ and $\theta$ were globally optimized to yield the best outcome, but not subject or day specific. The such optimized values could thus be used "out of the box" for any new subject. 
These two variations of the proposed algorithm were compared to the following 4 methods:

1. LDA: The basic, unprocessed output of the classifier and its resulting classification accuracy were used for baseline comparison with all other methods.

2. LDA-MV: The classic post-processing method of majority voting as introduced in [27] was applied as a moving average filter of the classification stream. The classifier decision was the one which was suggested the majority of times within a certain time window. In this study, a majority vote length of 9 was selected, since it showed to yield the best results in a preliminary investigation.

3. LDA-RJNM: This approach was proposed by Scheme et al. [52] and implemented as described in that publication, since it follows the same rational as motivated in this study, but purely focused on the instantaneous confidence value provided by the LDA: it was proposed to reject any classification made by the LDA which had a confidence value below 0.97 and relabel it to NM instead (RJNM - reject to no movement).

4. LDA-RJRM: This slight variation of LDA-RJNM employed the same methodology as LDA-RJNM, but instead of relabeling to NM, the last accepted class was used as output (RJRM - reject and remain in previous class). It was expected that this strategy would yield less discontinued prosthetic movements in an online application and was more directly comparable to the proposed MLP-ANN based system, which used the same relabeling strategy.

\subsubsection{Evaluations}

For the evaluations of the classification systems, two different metrics were used. The total accuracy $(t A c c)$ was calculated for describing the overall accuracy of each system as

$$
t A c c=\frac{\text { correct classifications }}{\text { total classifications }} \times 100
$$

In addition to this measure, the active accuracy $(a A c c)$ was considered:

$$
a A c c=\frac{\text { correct active classifications }}{\text { total active classifications }} \times 100
$$


where active classifications are such that they would result in a prosthetic action, i.e. classification to a class that causes the prosthesis to move. This is in contrast to a classification to NM, which causes the prosthesis to stop its current movement. The latter is the preferable type of classification error, since it does not cause any erroneous movements, thus making the prosthesis safer to use. However, accepting too many mis-classifications to NM would result in very discontinued, unsteady prosthetic movements. Therefore, a good control system should have very high aAcc while also maintaining a high $t A c c$ and those two measures should be considered in conjunction.

\subsubsection{Statistical Analysis}

A single factor analysis of variance (ANOVA) for repeated measures was performed to quantify the effects of the different algorithms investigated on the achieved classification accuracies. The factor was the algorithm used and had six levels (the six algorithms listed above) and subjects and days were treated as random variables. The statistical analysis was conducted with the hypothesis that there was no difference in the methods investigated. In case of a probability value of lower than 0.05 , this hypothesis had to be rejected and a significant difference in the performance of the algorithms was assumed. In the latter case, a Tukey-Kramer post hoc analysis [89,90] was conducted to assess pair-wise differences between algorithms. All averaged results are presented as the mean value \pm one standard deviation, calculated across all days and subjects. For all statistical analyses, the threshold for significance was set to $p<0.05$.

\subsection{Results}

Upon visual inspection, the output of the MLP-ANN effectively decreased in the presence of mis-classifications of the classifier. In case of continuously consistent predictions, the constant integration factor of $\beta(t)$ clearly reflected this measure of increased confidence by monotonously increasing $T I(t)$. The smoothing factor $\alpha$ worked efficiently for removing undesired small fluctuations while allowing also for fast responsive changes in case of decreasing $T I(t)$. The resulting trust index as computed from the output of the MLP-ANN along with the influences of $\alpha$ and the integration factor for $\beta(t)$ are shown for a representative example in Figure 3.3. 
(1)
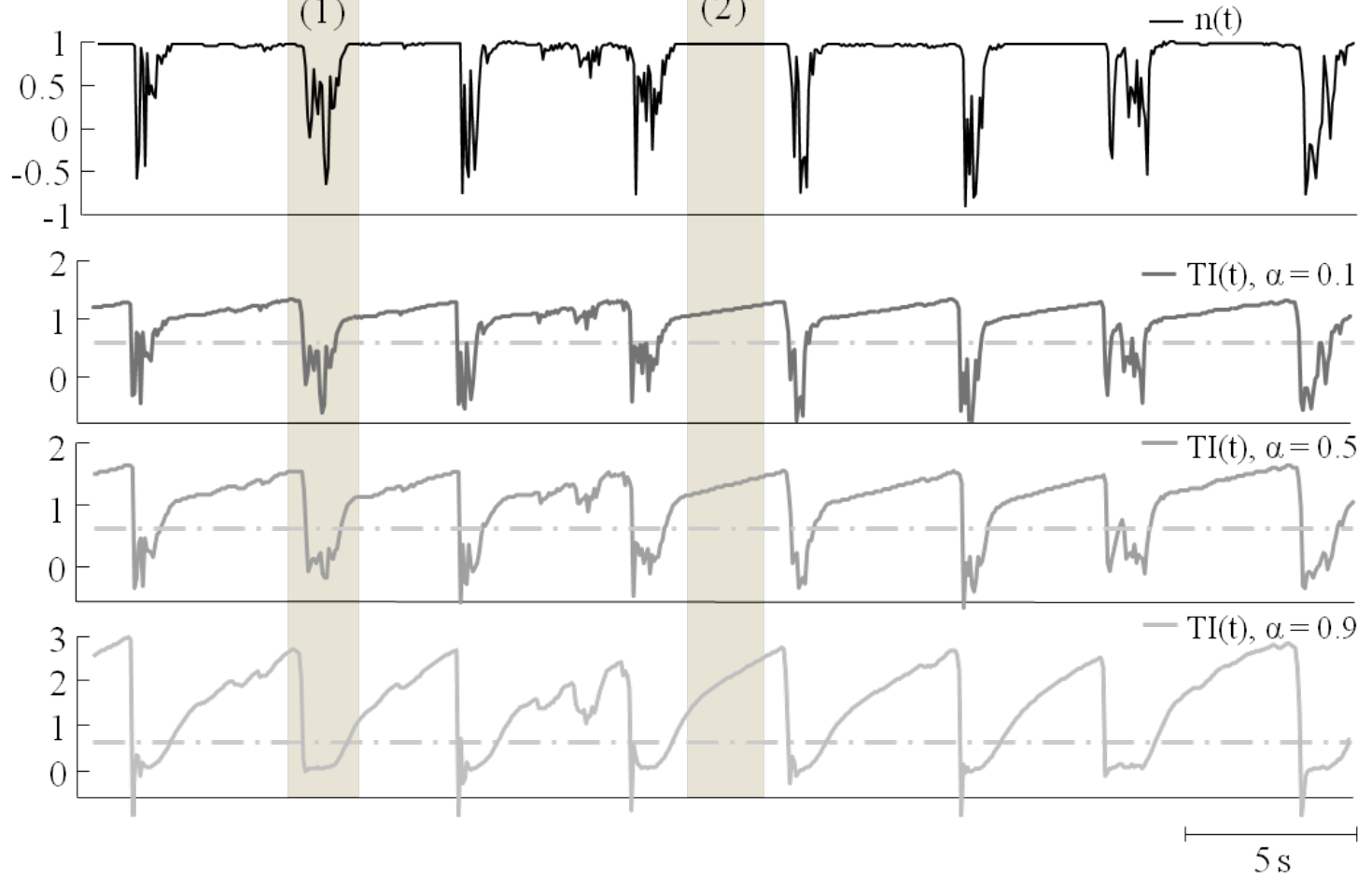

Figure 3.3: Examples of the trust index $T I(t)$ computed for the same network output $n(t)$ (top graph) with different smoothing factors $\alpha$ (graphs 2-4). In the highlighted section (1), the smoothing effect of $\alpha$ becomes apparent. In the plateau phases of the network output, such as highlighted in section (2), the effect of integrating $\beta(t)$ with a constant factor over time can be observed. It can be seen that choosing large values of $\alpha$ resulted in better artifact removal but also in an increased delay of the response. The same threshold value is shown as dash-dotted line in each $T I(t)$ graph for reference, below which the LDA classification decision was discarded. Reused and modified with permission [84] @2014 IEEE. 


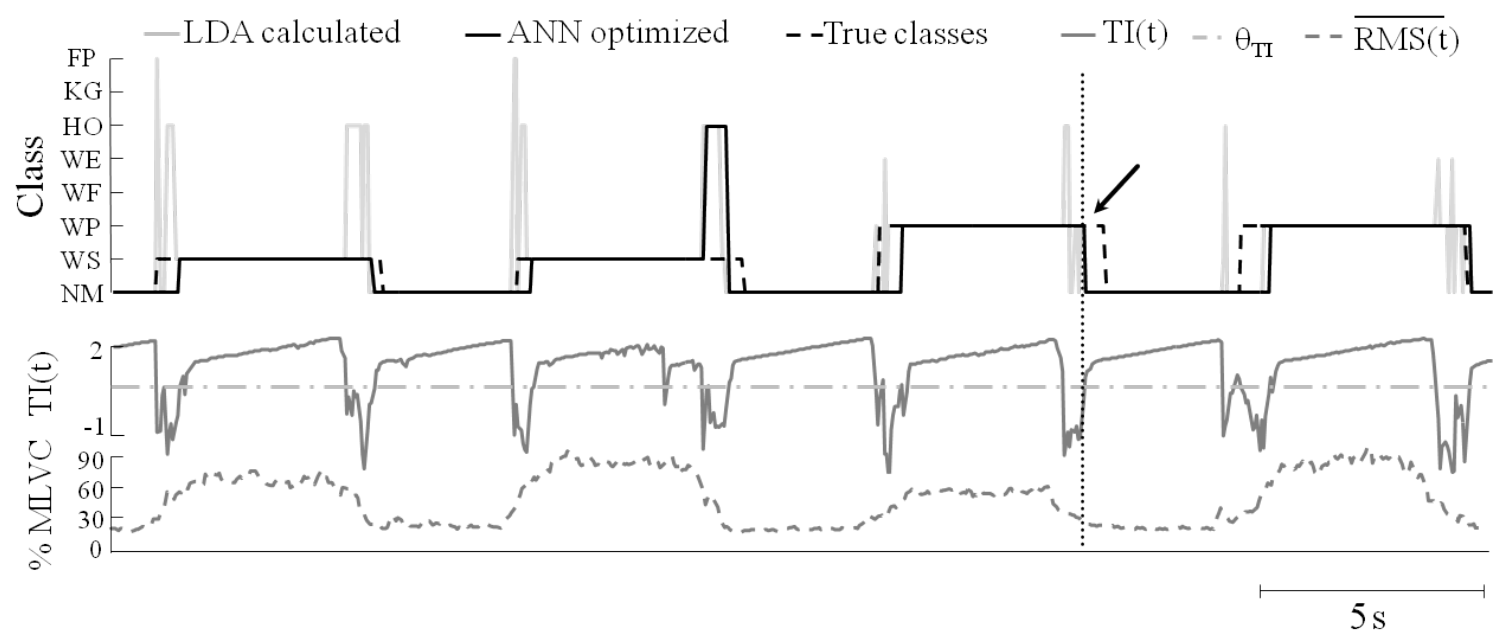

Figure 3.4: Exemplary exploitation of the trust index for removal of mis-classifications in a representative example. For this plot, $\alpha$ was set to 0.2 and $\theta$ to 0.61 , which were found to globally optimize the results among all subjects and days. It is apparent that many of the mis-classifications could effectively be removed. When inspecting the introduced time delay, it appears that the system at times predicted the relaxation state a few samples too late in the movement onset and before the prompt returned back to NM in the offset. However, if for example the area marked by the dotted vertical line and arrow is considered, observing the EMG RMS value reveals that the subject had already relaxed the contraction. This shows that the proposed method reacted timely to the actual performed class switch. Reused and modified with permission [84] (C2014 IEEE.

With this intuitively promising result, it was proceeded to apply the trust index to the removal of mis-classifications. An exemplary result of such a procedure is shown in Figure 3.4.

The observations described above and illustrated in Figure 3.3 were extended by evaluating the histogram of the $T I(t)$ values, grouped by correct and incorrect LDA decisions (Figure 3.5). This analysis demonstrated that at the majority of time instances, an incorrect LDA decision was accompanied by a $T I(t)$ value lower than the globally optimizing $\theta$ of 0.61 . Most correct LDA decisions also had a corresponding larger $T I(t)$ value. Note that some false positive detections (LDA was correct but $T I(t)<0.61)$ can be observed. However, in such a case, the algorithm would just relabel the LDA decision to the previously accepted class. Thus, while the selfcorrection mechanism would be incorrect in these cases, this would not necessarily result in wrong movement estimation of the entire system.

The exemplary, qualitative analyses discussed above and demonstrated in Figures 3.4 and 3.5 let the reader gain insight to the functioning principle of the proposed method. For a comprehensive quantitative analysis, the results of comparing all methods among each other, separated by subject groups, are presented in the following. 


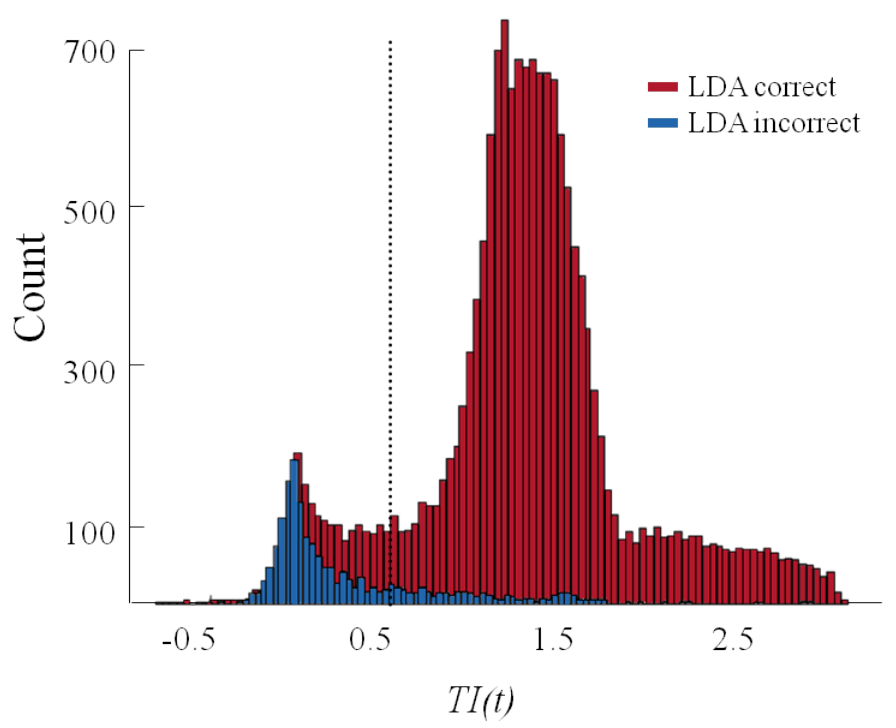

Figure 3.5: Histogram plot of the distributions of the trust index $T I(t)$, grouped by the correctness of the LDA classifier at time $t$ from an exemplary subject and day. It is shown that the majority of mis-classifications had a corresponding $T I(t)$ of lower than 0.61 (the globally optimizing threshold), whereas correct classifier decisions also corresponded to larger $T I(t)$ values. In a certain range $(\sim 0.35$ to $\sim 0.8)$ the system appeared insensitive to the exact choice of $\theta$, which was confirmed in the comprehensive optimization results for ANN-GO. Note that while the MLP-ANN output was limited to $[-1 ;+1]$, the filtered and integrated $T I(t)$ value could take values outside this interval. Reused and modified with permission [84] (C)2013 IEEE.

\subsubsection{Able-bodied subjects}

The parameter pair of $\alpha$ and $\theta$ was optimized by grid search to estimate the best overall result across all subjects and days. The pseudo-optimal values were found to be 0.2 and 0.61 for $\alpha$ and $\theta$, respectively. These values were used for all further analyses reported for ANN-GO in this study. In Figure 3.6 the result of the grid search is shown. The system was relatively robust towards the choice of the parameter values, since the resulting optimization plane had a flat characteristic.

The statistical evaluation revealed that the applied post processing method had a significant influence on the resulting classification accuracy of the entire system $\left(p<10^{-3}\right)$. It was thus proceeded to analyze the pairwise differences between the algorithms in a post hoc comparison.

The unprocessed classification results of the LDA classifier were regarded as the base line. The well-established majority voting method increased the unprocessed classification accuracy significantly by $2.3 \%$ tAcc and $3.4 \%$ aAcc $(p<0.05$ for both). The LDA-RJNM method as proposed in [52] performed very poorly in the assessment of the total accuracy $t A c c$ (worse than all other methods investigated with $p<10^{-3}$ ). 


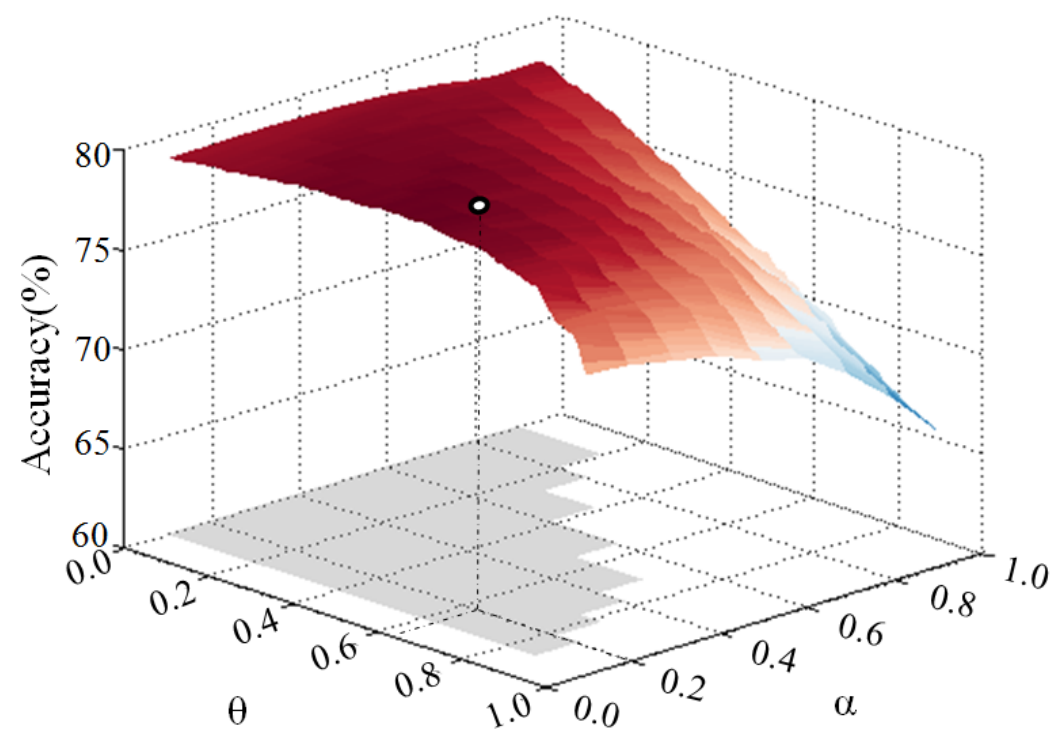

Figure 3.6: Visualization of the parameter optimization grid search of $\alpha$ and $\theta$. The optimal values were determined to be 0.2 and 0.61 , respectively. However, the resulting optimization plane was relatively flat, indicating robustness of the system towards the precise choice of the parameter values. The gray shaded area on the bottom of the plot indicates the parameter value pairs which result in improved results compared to the un-processed LDA output. Reused and modified with permission [84] (C)2014 IEEE.

Regarding the aAcc however, this method resulted in the second best performance achieved in this study (after ANN-IND), indicating that many of the classification decisions were relabeled to NM, while the majority of the not relabeled results were indeed correct classifications. This affected $t A c c$ negatively and $a A c c$ positively. The slight alteration proposed in the present study of not re-labeling to NM but to the previously accepted class (LDA-RJRM) significantly improved the $t$ Acc by $17.8 \%$ but decreased the $a A c c$ by $5.1 \%$ compared to LDA-RJNM. LDA-RJRM was better than the unprocessed LDA for $t A c c$ and $a A c c(p<0.05$ for both)

Regarding the proposed method, it was found that ANN-GO significantly outperformed all other investigated previously proposed methods in both $t A c c$ and $a A c c$, except for LDA-RJNM in $a A c c$ (difference $-1.2 \%, p=0.052$ ). Only ANN-IND resulted in better accuracies than ANN-GO $(+0.7 \%$ tAcc, $p>0.5$ and $+1.4 \%$ aAcc, $p<0.05)$.

In general, the achieved classification accuracies were relatively low $(<85 \%$ tAcc and $<95 \%$ aAcc) compared to other offline studies. This is likely attributable to the various non-stationarities included in the data set of this study, including session to session transfer effects across days and the exclusion of NM from the active classification. In order to assess the value of the proposed system not only under the aspect of 
Mean \pm std accuracy, averaged over able-bodied subjects and days

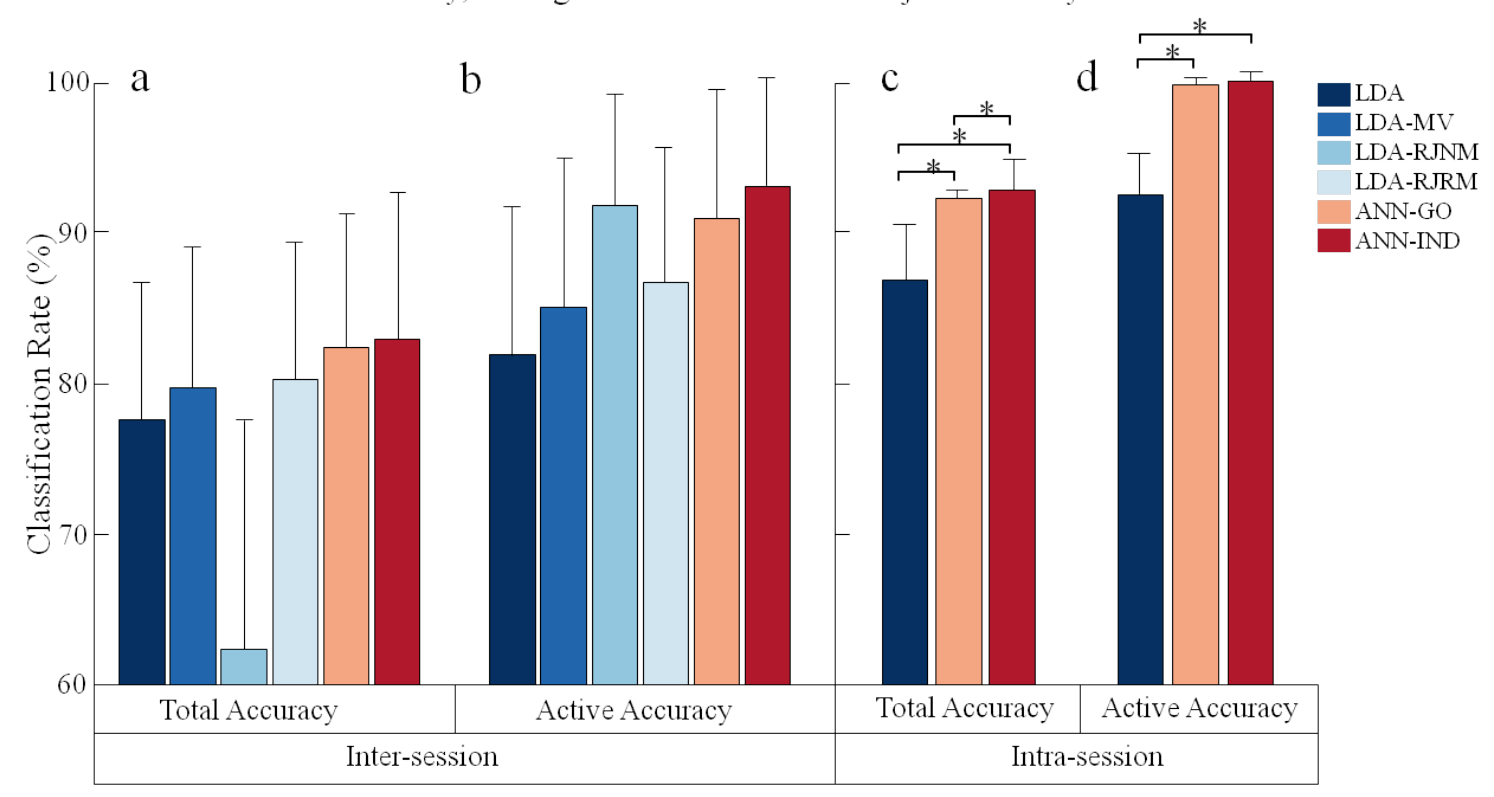

Figure 3.7: Comparison of all investigated algorithms, showing the $t A c c(\mathrm{a}, \mathrm{c})$ and $a A c c(\mathrm{~b}, \mathrm{~d})$ for able-bodied subjects, when training and testing set were recorded with one day difference $(\mathrm{a}, \mathrm{b})$ or stemmed from the same session $(\mathrm{c}, \mathrm{d})$. The proposed method performed significantly better than the baseline, both in tAcc and aAcc. LDA-RJNM performed significantly worse than all other methods in tAcc but yielded very good results in aAcc. In intra-session testing, the active accuracy reached close to $100 \%$. For details of pair wise differences of methods in inter-session comparisons see Table 3.1. For intra-session, ${ }^{*}$ denotes $p<0.05$. Reused and modified with permission [84] (C)2014 IEEE.

these non-stationarities but also within a session of classifier testing, an intra-session analysis was conducted to compare the performance gain with the proposed method over the unprocessed LDA accuracy. Both in $t A c c$ and $a A c c$ the two variants of the proposed method significantly outperformed the base line accuracy. The ANN based post-processing yielded an accuracy gain of $>5 \%$ in all comparisons to LDA in both accuracy types investigated. An aAcc close to $100 \%$ correct classifications were achieved, demonstrating the benefit of the proposed system not only under the presence of non-stationarities.

The results of the able-bodied subject group are summarized in Figure 3.7. Note that for clarity, all pairwise comparisons of significance are not shown in Figure 3.7 for the inter-session comparisons but are highlighted in bold-face font in Table 3.1 together with the exact amount of classification accuracy difference. 
Table 3.1: Detailed summary of differences between algorithms for able-bodied subjects. Positive (negative) values in cells represent improvement (deterioration) of the method in that column with respect to the method in that row, separated by $t A c c$ and $a A c c$. Bold values indicate significant differences (repeated measures ANOVA, post hoc Tukey-Kramer comparison, $p<0.05$ ). Reused and modified with permission [84] (C)2013 IEEE.

\begin{tabular}{rcccccccccc}
\hline & \multicolumn{2}{c}{ LDA-MV } & \multicolumn{2}{c}{ LDA-RJNM } & \multicolumn{2}{c}{ LDA-RJRM } & \multicolumn{2}{c}{ ANN-GO } & \multicolumn{2}{c}{ ANN-IND } \\
& tAcc & aAcc & tAcc & aAcc & tAcc & aAcc & tAcc & aAcc & tAcc & aAcc \\
\hline LDA & $\mathbf{2 . 2 9}$ & $\mathbf{3 . 3 5}$ & $\mathbf{- 1 5 . 1}$ & $\mathbf{9 . 4 8}$ & $\mathbf{2 . 6 6}$ & $\mathbf{4 . 3 4}$ & $\mathbf{4 . 7 5}$ & $\mathbf{8 . 2 4}$ & $\mathbf{5 . 4 6}$ & $\mathbf{9 . 6 6}$ \\
LDA-MV & & & $\mathbf{- 1 7 . 3 9}$ & $\mathbf{6 . 1 3}$ & 0.37 & 0.99 & $\mathbf{2 . 4 6}$ & $\mathbf{4 . 8 9}$ & $\mathbf{3 . 1 7}$ & $\mathbf{6 . 3 1}$ \\
LDA-RJNM & & & & & $\mathbf{1 7 . 7 5}$ & $\mathbf{- 5 . 1 4}$ & $\mathbf{1 9 . 8 5}$ & -1.23 & $\mathbf{2 0 . 5 6}$ & 0.19 \\
LDA-RJRM & & & & & & & $\mathbf{2 . 0 9}$ & $\mathbf{3 . 9}$ & $\mathbf{2 . 8 0}$ & $\mathbf{5 . 3 2}$ \\
ANN-GO & & & & & & & & & 0.71 & $\mathbf{1 . 4 2}$ \\
\hline
\end{tabular}

\subsubsection{Amputee subjects}

The same evaluations as performed for the able-bodied subjects were carried out for the amputee subjects. Although the patient group achieved lower absolute recognition accuracies, the improvements achieved by the the investigated post-processing methods showed the same trends as in the control group.

As with the first subject group, the statistical analysis revealed a significant influence of post-processing method on the recognition rate of the entire system $\left(p<10^{-3}\right)$ and the pairwise method comparisons were performed. LDA achieved an average $t A c c$ of $59.2 \pm 15 \%$ and $a A c c$ of $63.7 \pm 18.4 \%$. These values were regarded as the baseline for all other methods. Majority voting again resulted in a slight but not significant increase of accuracy by $2.4 \%$ and $3.28 \%$ for $t A c c$ and $a A c c$, respectively. LDA-RJNM showed the same tendencies as described above: It resulted in a decrease of the overall classification accuracy $t A c c$ of $25.6 \%$, but performed excellently with respect to $a A c c$ (increase of $20.4 \%$ compared to the base line, both comparisons $p<10^{-3}$ ). In $a A c c$, this method was only outperformed by ANN-GO and ANN-IND, by $1.2 \%$ and $11.1 \%$ respectively. LDA-RJRM performed significantly better in $t$ Acc than LDA-RJNM but also worse in $a A c c$. The methods which performed best were again consistently ANN-GO and ANN-IND. These two methods outperformed all other methods investigated in this study. In $t A c c$ they improved the baseline classification accuracy by $4.6 \%$ and $5.9 \%$ and in $a A c c$ by $21.6 \%$ and $31.5 \%$, respectively (all improvements $p<0.05)$.

Also in the within-session control evaluation, the proposed method resulted in significantly improved recognition rates. In this scenario, the $a A c c$ closely approached 


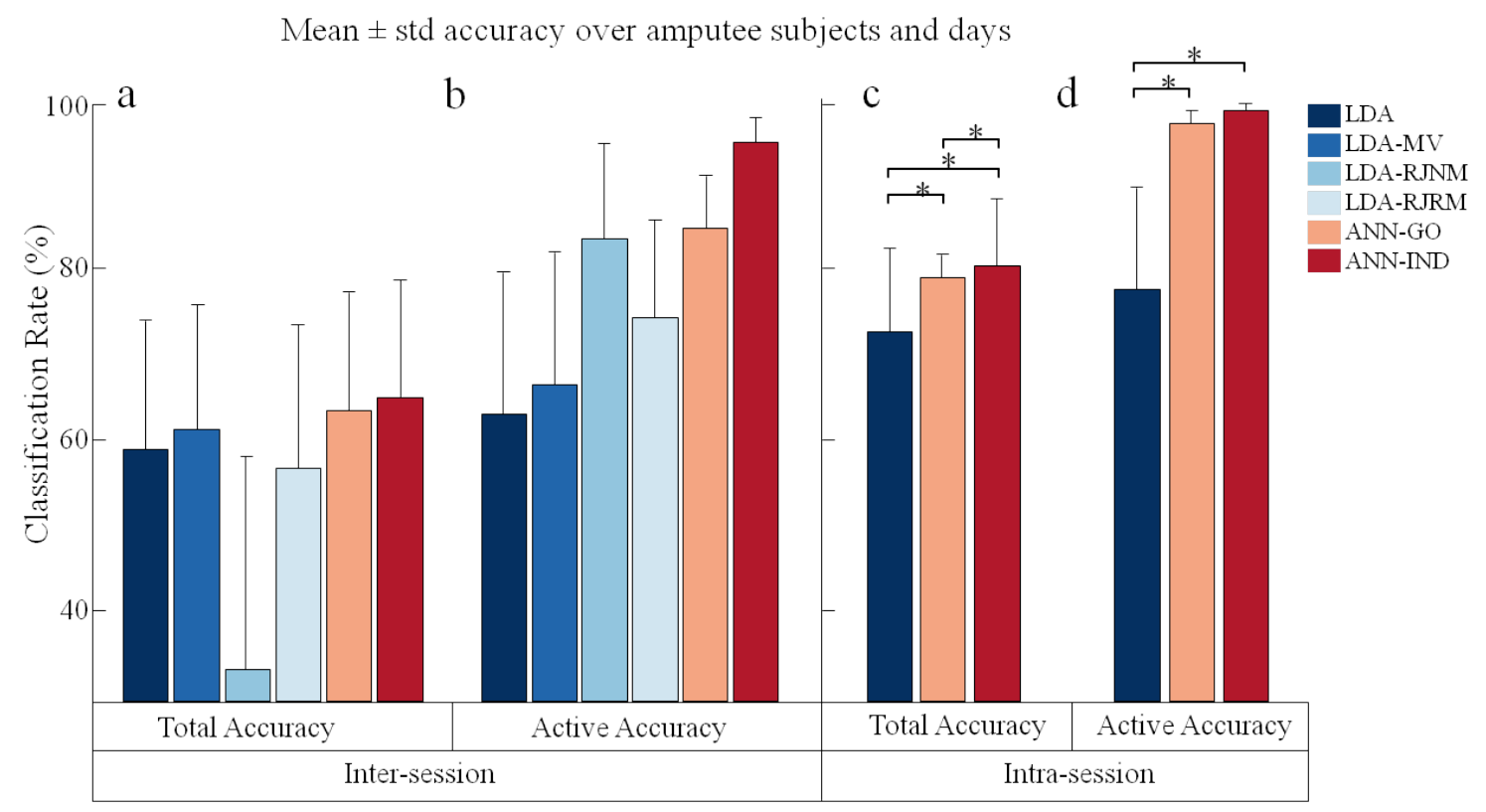

Figure 3.8: Comparison of all investigated algorithms, showing the $t A c c(\mathrm{a}, \mathrm{c})$ and $a A c c(\mathrm{~b}, \mathrm{~d})$ for amputee subjects, when training and testing set were recorded with one day difference $(\mathrm{a}, \mathrm{b})$ or stemmed from the same session $(\mathrm{c}, \mathrm{d})$. The proposed method performed significantly better than the baseline, both in tAcc and aAcc. LDA-RJNM performed significantly worse than all other methods in tAcc but yielded very good results in aAcc. In intra-session testing, the active accuracy reached close to $100 \%$. For details of pair wise differences of methods in inter-session comparisons see Table 3.2. For intra-session, ${ }^{*}$ denotes $p<0.05$. Reused and modified with permission [84] (C)2014 IEEE.

100\%. Therefore, this method yielded significant improvements for both inter-session and intra-session testing in amputees compared to the baseline LDA classification accuracy.

The results of the amputee subject group are summarized in Figure 3.8. Note that the comparisons of significance are not shown in Figure 3.8 for the inter-session comparisons but are highlighted in bold-face font in Table 3.2. In this table also the pairwise classification accuracy gains are shown for each compared method pair.

\subsubsection{Time accuracy}

One important consideration in the analysis of post-processing methods is the induced time delay for a class change to be accepted. Two types of delays can be examined: the delay which occurs for a movement to start (i.e. transition delay from NM to the active class) and the delay of an active classification returning back to NM. It was found that in the context of this study, both types of delay occurred with approximately the same frequency and duration. They are thus summarized as "time accuracy" in 
Table 3.2: Detailed summary of differences between algorithms for amputee subjects. Positive (negative) values in cells represent improvement (deterioration) of the method in that column with respect to the method in that row, separated by $t A c c$ and $a A c c$. Bold values indicate significant differences (repeated measures ANOVA, post hoc Tukey-Kramer comparison, $p<0.05$ ). Reused and modified with permission [84] (C)2014 IEEE.

\begin{tabular}{rcccccccccc}
\hline & \multicolumn{2}{c}{ LDA-MV } & \multicolumn{2}{c}{ LDA-RJNM } & \multicolumn{2}{c}{ LDA-RJRM } & \multicolumn{2}{c}{ ANN-GO } & \multicolumn{2}{c}{ ANN-IND } \\
& tAcc & aAcc & tAcc & aAcc & tAcc & aAcc & tAcc & aAcc & tAcc & aAcc \\
\hline LDA & 2.44 & 3.28 & $\mathbf{- 2 5 . 6 4}$ & $\mathbf{2 0 . 3 8}$ & -2.22 & $\mathbf{1 1 . 0 5}$ & $\mathbf{4 . 6 0}$ & $\mathbf{2 1 . 5 8}$ & $\mathbf{5 . 9 2}$ & $\mathbf{3 1 . 5 1}$ \\
LDA-MV & & & $\mathbf{- 2 8 . 0 9}$ & $\mathbf{1 7 . 1 0}$ & $\mathbf{- 4 . 6 6}$ & $\mathbf{7 . 7 7}$ & 2.16 & $\mathbf{1 8 . 3 0}$ & 3.48 & $\mathbf{2 8 . 2 3}$ \\
LDA-RJNM & & & & & $\mathbf{2 3 . 4 3}$ & $\mathbf{- 9 . 3 3}$ & $\mathbf{3 0 . 2 5}$ & -1.20 & $\mathbf{3 1 . 5 7}$ & $\mathbf{1 1 . 1 3}$ \\
LDA-RJRM & & & & & & & $\mathbf{6 . 8 2}$ & $\mathbf{1 0 . 5 2}$ & $\mathbf{8 . 1 4}$ & $\mathbf{2 0 . 4 6}$ \\
ANN-GO & & & & & & & & 1.32 & $\mathbf{9 . 9 3}$ \\
\hline
\end{tabular}

this study and are investigated together. The delays are reported with respect to the unprocessed LDA class transitions to avoid bias by the subjects' reaction times to the movement prompts (assuming that LDA recognized NM correctly for the vast majority of cases, which was shown to be the case as described above).

The median time accuracy of the MLP-ANN based correction mechanism was found to be $200 \mathrm{~ms}$ for ANN-GO and $250 \mathrm{~ms}$ for ANN-IND in able-bodied subjects and $300 \mathrm{~ms}$ for both method variants in amputee subjects and was slightly skewed towards shorter delays. The other investigated methods behaved similarly or were slightly faster, but none of the methods managed to have a better time accuracy than 100-175 ms, which was found to be the threshold for noticeable delay in [91].

\subsection{Study discussion and conclusion}

In the presented study a novel post-processing method for EMG signal classification for prosthetic control has been introduced. While in this study the base classifier was limited to LDA, many other classification methods such as kNN and SVM could be combined with the proposed method. The only requirement is that the classifier produces an estimate of the reliability of its estimation (e.g. minimal distance to training samples in $\mathrm{kNN}$ or distance to separation hyperplane in SVM). The development of the system was motivated by observations made in previous studies that mis-classifications often occurred during dynamic contraction phases and movement transitions, accompanied by low classifier confidence values. It could be shown that the proposed method effectively improved the classification accuracy in these situations (Figure 3.4). 


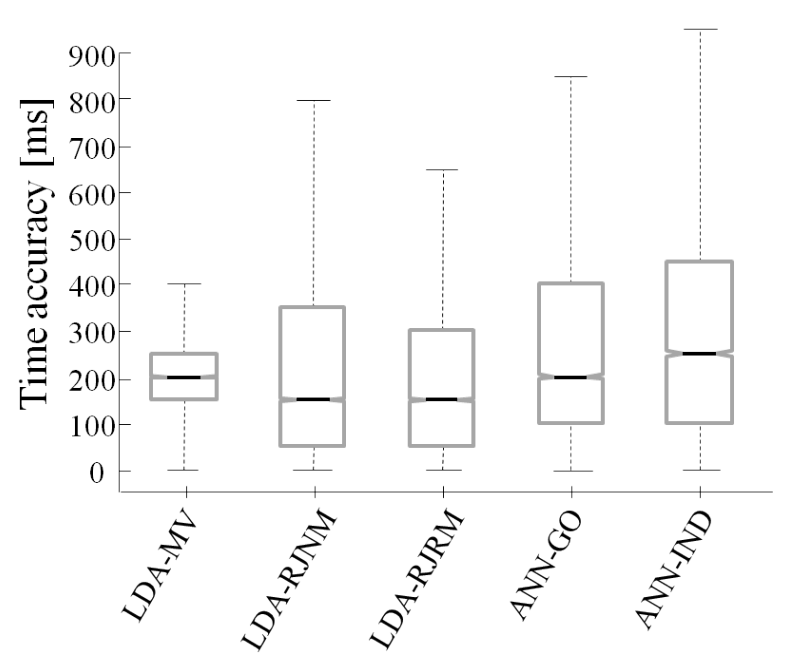

(a) Time accuracy able-bodied subjects

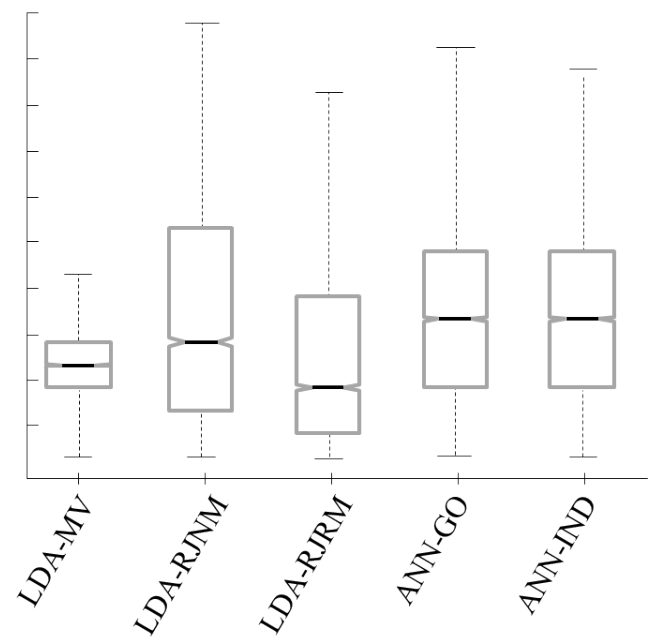

(b) Time accuracy amputee subjects

Figure 3.9: Results of time accuracy analysis of each algorithm for (a) able-bodied and (b) amputee subjects. Any time deviation from the raw classifier output was counted (50 ms time window increment from one classification to the next). It was found that, on average, all algorithms had a time accuracy equal to or shorter than $300 \mathrm{~ms}$, but the proposed method did not result in any improvement in this aspect with respect to all other methods. Reused and modified with permission [84] (C) 2014 IEEE.

For a thorough investigation and analysis of the proposed method, a challenging data set was recorded with able-bodied and amputee subjects. The data set contained contractions of dynamic movements with weak to strong plateaus. Furthermore, the training and testing sets of the classification were recorded in sessions of different days. This inevitably resulted in different electrode-skin impedance, electrode shifts etc. as described in the introduction of this chapter. These non-stationarities were included to enhance the clinical relevance of the investigated methods, as they would naturally occur during routine usage of a prosthesis by an amputee. This also explains the relatively low achieved classification accuracies, which were often reported to be $>95 \%$ in studies not containing such non-stationarities [28]. The focus on clinical relevance in the present study was further extended by individually manufactured test prosthetic sockets custom made by a prosthetist for each amputee subject.

In this realistic setup the proposed approach significantly outperformed all other methods in aAcc in both subject groups, except for LDA-RJNM which performed slightly better than ANN-GO in able-bodied subjects. In this particular comparison however it is important to consider the combined results of $t A c c$ and $a A c c$. Theoretically, a trivial system always predicting NM could achieve 100\% aAcc. Therefore, 
this measure has to be considered in conjunction with $t A c c$, where such a system would yield $0 \%$ (note that NM was not actively classified). Under this viewpoint, both ANN-GO and ANN-IND outperformed indeed all other methods investigated, since they simultaneously yielded high $t A c c$ and $a A c c$. In amputees, a performance gain of up to $30 \%$ could be demonstrated.

The effectiveness of the proposed method was compared to the base line of unprocessed classification and to 3 other post-processing methods. Two of these methods (LDA-MV, LDA-RJNM) were previously proposed in literature and the third (LDARJRM) was a slight alteration of one of them to investigate the influence of relabeling strategy. In direct comparison, LDA-RJRM considerably outperformed LDA-RJNM in the total classification accuracy $t A c c(+17.8 \%)$, but performed $5.1 \%$ worse when mis-classifications to NM were not regarded as errors $(a A c c)$. This indicates that in an online application the resulting control system with LDA-RJRM would permit more fluent, continuous prosthetic movements but with slightly more erroneous activations than LDA-RJNM. In this offline study it cannot be concluded which of the two methods would result in the preferable system during online control.

In this study the time accuracy was investigated as a separate measure. It was found that the proposed method did not introduce shorter delays than the other methods. The maximum latency described was $300 \mathrm{~ms}$, corresponding to 6 time windows delay. It might be speculated that if the increment between windows would be reduced to e.g. $30 \mathrm{~ms}$, the time delay could be reduced to around $180 \mathrm{~ms}$, which is almost below the threshold of noticeable delay [91]. However, this assumption would have to be confirmed in a dedicated evaluation. Lastly it is worth mentioning that heuristic rules, such as preferring switches to NM, could decrease the time latency for ending a movement. However, such attempts were outside the scope of this study.

As opposed to adaptive algorithms [48,85,92], the proposed self-correction system of this study does not require recording of additional training data. The same data set as used for training the base classifier was used, which is also important in a clinical setup.

In conclusion, a novel method of self-correction for a classifier has been introduced and its effectiveness evaluated in a challenging data set recorded with able-bodied and amputee subjects. The highly significant improvements achieved in this study foster expectations that the observed effects would also have beneficial influences on the real-time control of a physical prosthesis. However, in this study the focus was 
laid on the introduction of the system and limited to offline evaluations, facilitating the comprehensive evaluation of many methods as it would not have been possible in an online study. The promising results achieved in this study are therefore to be proven relevant for a clinical use for improving EMG based pattern recognition for intuitive upper limb prosthetic control in an online, real-time control study. 


\section{A novel multi-class proportional estimator}

In the previous two studies presented, the focus was put on improving the more traditional approach of classification of EMG signals for myoelectric prosthetic control. In this and the following study, another type of machine learning method - regression - will be explored. Regression methods have the intrinsic advantage of estimating proportional output, which can directly be used to drive a prosthesis in a smooth way. In classification, only the currently active movement type can be determined, but a proportional control value has to be extracted separately, which is not necessarily straightforward [53]. A further advantage of regression models is that they can estimate the activation of several DOF simultaneously, potentially allowing more natural and fluent motions. However, due to their parallel nature of estimation, it is sometimes difficult to selectively activate only one function while not activating any of the other. This was for example discussed in [58].

Therefore, the development of a novel regression method capable of suppressing wrong movements appeared desirable. The goals and basic ideas, which will be presented in this chapter, are in line with that introduced in Chapter 3 - applied to regression. However, the same idea as presented in Chapter 3 was not directly applicable for regression purposes, since it relied on the relabeling of movements in case of uncertainty, which is not appropriate for continuous force estimation. Furthermore, in general regressors do not output a measure of confidence for an estimation and they do not suffer from transient movement phases to the extent classifiers do (which was one of the premises for the history based ANN correction). Therefore a novel strategy was pursued to substantially increase robustness of regression based myoelectric prosthetic control.

CSP is a spatial filter routinely used in electroencephalography (EEG) analysis, where 
it is used to enhance the low signal-to-noise ratio of EEG signals. This method has also been used as spatial sEMG raw signal filter in [93]. In its classic application, CSP is therefore used as a spatial filter for raw signals in conjunction with high-density signal recordings, containing both temporal and spatial information. The novel idea conceived in the present study with only 8 channels was to apply the same technique to features extracted from the EMG signals rather than the raw signals themselves. This resulted directly in a novel proportional movement estimator, as will be described in the following sections.

A further difference of this study with respect to the ones previously described in this thesis is that from now on the main focus will be put on online evaluations of direct control of a physical hand prosthesis. This approach is by far more expressive and allows for much more direct estimation of the gained benefit of the investigated $\operatorname{method}(\mathrm{s})$ for the target application in amputee users. The drawback of this evaluation method is that it does not allow for the comparison of a multitude of different control strategies due to time and fatigue constraints of the subjects. Nevertheless, in the opinion of the author this limitation is outweighed largely by the functional insights gained in such an assessment and its more direct transferability to the clinical relevance. For this reason, offline analyses will be very limited in the following evaluations and functional real-time tests will be emphasized.

The concept of this study, as well as the results have been submitted for publication in similar form as presented here by me as first author in [94] and parts of it in [95]. Therefore, text or results reproduced from this manuscript are not cited explicitly in the following. All figures and tables were reproduced with permission.

\subsection{Methods and procedures}

In this section, first the development of a novel multi-class proportional estimator based on the CSP method (CSP-PE) is detailed. Subsequently, a test protocol is defined which allows for the systematic evaluation of the derived control system based on CSP-PE. The evaluation will be based on online measurements and comparisons to state-of-the-art control schemes will be made. 


\subsubsection{Common spatial patterns proportional estimator (CSP-PE)}

The CSP method implements a spatial filter for multi-channel recordings. Originally, it has been applied in two-class classification tasks of EEG analysis and brain computer interfacing. In this domain, it extracts features from the raw signal recordings which are optimized to maximally discriminate between the data of two classes. It is therefore a supervised algorithm that requires a priori information for training. It was first described in 1991 by Koles [96] and the term CSP was coined by MuellerGerking et al. in 1999 [97]. It was soon adopted by many research groups and a considerable number of variations to the original scheme has been proposed (see e.g. $[98,99,100,101]$ and references therein).

In order to find features which maximize the distance between two classes, the raw input signals $\boldsymbol{x} \in \mathbb{R}^{c}$ are transformed by a linear transformation $W \in \mathbb{R}^{c \times d}$ to a $d$-dimensional vector $\boldsymbol{y} \in \mathbb{R}^{d}$ in a space that is characterized by maximal variance for data of the first class while minimizing it for data from the second class (in the spatial filtering context, the values in $\boldsymbol{y}$ are called components).

$$
\begin{aligned}
\boldsymbol{y} & =W^{T} \boldsymbol{x} \\
\operatorname{var}(\boldsymbol{y}) & =E\left[\boldsymbol{y} \boldsymbol{y}^{T}\right]=W^{T} E\left[\boldsymbol{x} \boldsymbol{x}^{T}\right] W
\end{aligned}
$$

where $E[\cdot]$ is the expectation operator. Assuming that $\boldsymbol{x}$ and $\boldsymbol{y}$ are drawn from centered distributions, (4.2) can be calculated for a series of observations as

$$
\operatorname{var}(y)=W^{T} \hat{\Sigma} W
$$

where $\hat{\Sigma}$ is the empiric covariance matrix of $\boldsymbol{x}$.

For obtaining a transformation matrix $W$, which simultaneously maximizes the variance for data of class 1 and minimizes it for data of class 2 , it is suitable to optimize the ratio of variances as described in (4.3). The resulting quotient is known as the generalized Rayleigh quotient:

$$
W:=\underset{W}{\arg \max } \frac{W^{T} \hat{\Sigma}_{1} W}{W^{T} \hat{\Sigma}_{2} W}
$$




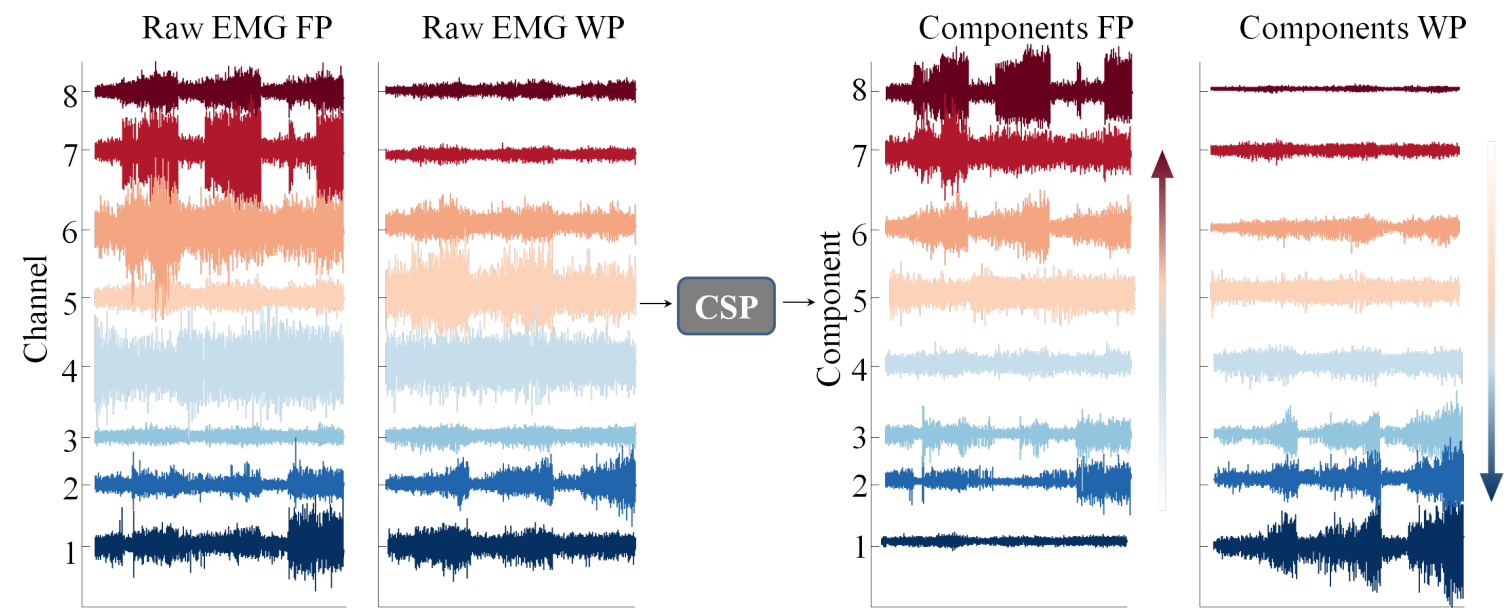

Figure 4.1: Exemplary result of applying CSP filter to 8 EMG signals. Note that while the activities of the raw EMG signals are not ordered, the CSP components are ordered (for class FP the first component has most variance and the last the smallest and vice verse for class WP, as indicated by the arrows).

where $\hat{\Sigma}_{i}$ represents the empiric covariance matrix of the data from class $i$. The optimization procedure of (4.4) using the Lagrangian method is equivalent to the one described in Chapter 1, Section 1.5.1, Equations (1.8) through (1.13). Their similar calculation indicates the close relationship of the CSP and LDA methods. The columns of $W$ contain the spatial filters, and the first column is the one which maximizes the variance for data of class 1 and the last column for class 2. This is well illustrated for high-density EMG recordings in [93] and for 8 channels in Figure 4.1.

The close resemblance of CSP and LDA and the fact that LDA is commonly applied to feature data, somewhat inspired the application of the CSP method to feature data in this study for the derivation of CSP-PE. After determining the projection matrix $W,(4.2)$ simply performs a linear combination of the values in $\boldsymbol{x}$ with the coefficients of the columns in $W$. Hence, when choosing $\boldsymbol{x}$ to be a feature vector with elements proportional to the amplitude of the recorded EMG and thus the exerted force, the output of this linear combination is as well proportional to the force. Furthermore, the optimization criterion as described above provides maximally distinct output for data of different classes. Therefore, the spatial filters obtained from the CSP optimization contain larger coefficients for features that are distinctly activated between the two classes and those features, which have overlapping activations, are weighted with smaller coefficients. Because of these properties, CSP-PE theoretically yields an estimator for EMG driven myoelectric control with the favorable properties of a 
regression method while maintaining high discriminability between movements.

Due to the utilization of force related feature values rather than the raw EMG signals, the assumption of centered data (zero mean) made in (4.3) was no longer valid. Therefore, instead of using the empiric covariance matrix, the empiric correlation matrix was used. In order to extend the described method to a multi-class problem of $m$ classes, the well-known one-versus-one scheme was applied.

\section{Application of the CSP-PE method}

For the remainder of this section, the following taxonomy is defined. Considering the transformation of two vectors $\boldsymbol{x}_{i}$ and $\boldsymbol{x}_{j}$ with the same column $\boldsymbol{w}$ of $W$

$$
\begin{aligned}
y_{i} & =\boldsymbol{w}^{T} \boldsymbol{x}_{i} \\
y_{j} & =\boldsymbol{w}^{T} \boldsymbol{x}_{j}
\end{aligned}
$$

where $y_{i / j}$ are scalars, then the terminology that class $i$ is winning this pair-wise CSP competition if $y_{i}>y_{j}$. Since the CSP transformation is designed to yield large values for one class and small values for the other class of that comparison, this terminology is considered to be intuitive. Further, the ratio of $\frac{y_{i}}{y_{j}}$ is termed the contrast by which class $i$ won this competition against class $j$.

In order to compute the result of all $\frac{m^{2}-m}{2}$ one-versus-one class competitions in a single matrix-vector multiplication, the first and last column of each individual CSP competition were compiled in one matrix $W_{\text {comp }} \in \mathbb{R}^{c \times m^{2}-m}$.

$$
W_{c o m p}=\left[w_{12}, w_{21}, w_{13}, w_{31}, \ldots, w_{(m-1) m}, w_{m(m-1)}\right]
$$

where $w_{i j}\left(w_{j i}\right)$ are the weight vectors which maximize the output for the data of class $i(j)$ while minimizing it for class $j(i)$ and were obtained from computing the CSP weight matrix between the classes $i$ and $j$. Therefore, $W_{\text {comp }}$ contains all those weight vectors that maximize the contrast between all class pairs with each class winning each comparison exactly once.

For the estimation of a newly observed feature vector $\boldsymbol{x}_{o b s}$, the multiplication with 
$W_{\text {comp }}^{T}$ yields a vector of competition results, $\boldsymbol{y}_{\text {comp }} \in \mathbb{R}^{m^{2}-m}$ :

$$
\boldsymbol{y}_{\text {comp }}=W_{c o m p}^{T} \boldsymbol{x}_{o b s}
$$

with elements

$$
\boldsymbol{y}_{\text {comp }}=\left[y_{12}, y_{21}, y_{13}, y_{31}, \ldots, y_{(m-1) m}, y_{m(m-1)}\right]
$$

In (4.8), each element of $y_{\text {comp }}$ is the result of the inner product between the filter weights and the feature vector.

For illustration, consider an example of a $m=4$ class problem: $\boldsymbol{y}_{\text {comp }}$ would be composed of the elements $\left[\begin{array}{llllllllllll}y_{12} & y_{21} & y_{13} & y_{31} & y_{14} & y_{41} & y_{23} & y_{32} & y_{24} & y_{42} & y_{34} & y_{43}\end{array}\right]$. Thus, e.g. class 3 would be present in the $\frac{16-4}{2}=6$ competition activations $\left(\begin{array}{lll}y_{13} & y_{31} & y_{23}\end{array}\right.$

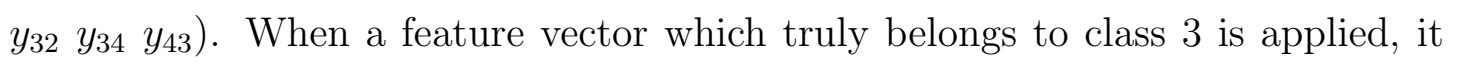
should yield large activation values in $y_{31}, y_{32}$ and $y_{34}$ and small activation values in $y_{13}, y_{23}$ and $y_{43}$. The contrasts by which class 3 wins its CSP competitions are computed as $\frac{y_{31}}{y_{13}}, \frac{y_{32}}{y_{23}}$ and $\frac{y_{34}}{y_{43}}$.

Finally, the activation $\alpha_{i}$ of a class $i \in[1 \ldots m]$, which is in competition against all other classes $j \in[1 \ldots m], j \neq i$, is computed by multiplying the minimum of its competition results with the maximum of the contrasts by which it won:

$$
\alpha_{i}:=\min _{j} y_{i j} \cdot \max _{j} \frac{y_{i j}}{y_{j i}}
$$

where the winning contrasts $\frac{y_{i j}}{y_{j i}}$ of class $i$ are normalized to sum up to 1 across all $j$

$$
\sum_{j} \frac{y_{i j}}{y_{j i}}=1
$$

The winning contrasts can thus be regarded as probability measures for the correctness of the activation estimation obtained from $\min _{j} y_{i j}$. 

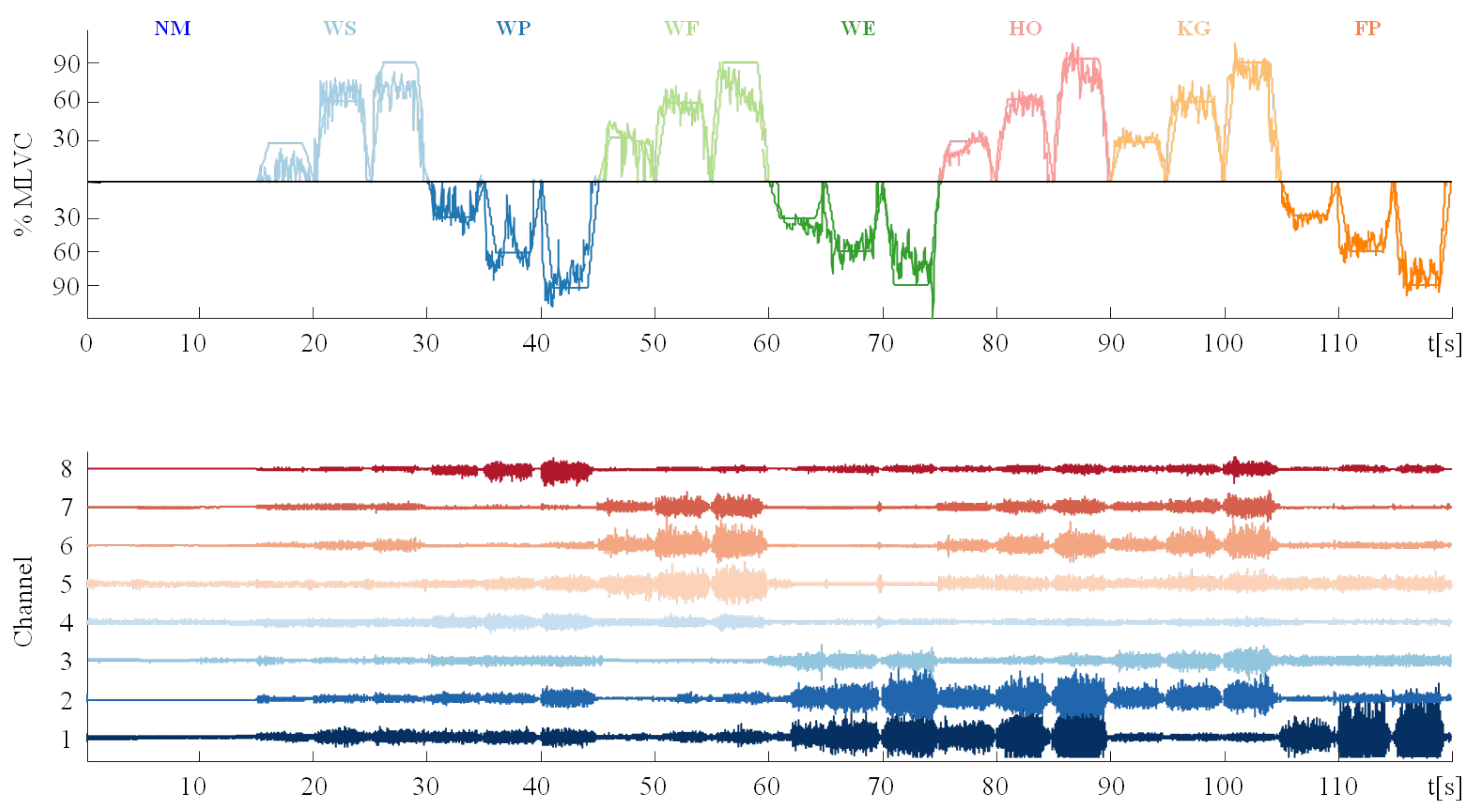

Figure 4.2: Exemplary result of applying CSP-PE (upper part) to EMG signals (lower part). For the activations, positive values represent movement in one direction (e.g. supination), negative values in the opposite direction (e.g. pronation) - together representing one DOF. The prompted movements are shown as straight lines, the estimation results are plotted on top.

Continuing the example from above, the activation of class $3, \alpha_{3}$, would be calculated by taking the minimum of $\left[y_{31}, y_{32}, y_{34}\right]$ and multiplying this value by the maximum of $\left[\frac{y_{31}}{y_{13}}, \frac{y_{32}}{y_{23}}, \frac{y_{34}}{y_{43}}\right]$, where these three numbers would have to be normalized to sum up to 1.

The raw outputs of the regression were rescaled, so that when re-applying the training data to the obtained estimator, a maximum of $100 \%$ movement speed in each DOF was achieved. An exemplary output of the CSP-PE method is shown in Figure 4.2.

\section{How CSP-PE works}

Due to considering only the minimum of all competition results of a particular class in (4.9), this class has to win all the CSP competitions against all other classes to be attributed a large activation value $\alpha$. If it loses at least one of the competitions, it will not be able to achieve a high activation output. Furthermore it has to win each of the competitions with a large contrast, otherwise its output will be reduced as well.

The combination of these two factors in (4.9) make this approach very selective and 
thus suitable for robust, reliable and safe operation of a prosthesis, since it minimizes the risk of wrong prosthetic activations. Additionally, when using force sensitive features, in case of confident estimation its output is proportional to the exerted force and thus also allows for smooth prosthesis operation. In summary, the derived approach promises to allow for very reliable, proportional control of myoelectric driven prostheses. The testing of this hypothesis in real life test scenarios with able-bodied and amputee subjects is described in the following sections of this chapter. Its performance is compared to two other control schemes, as described next.

\subsubsection{Compared methods}

In order to compare the newly developed method, two state-of-the-art reference control methods were equally tested.

\section{LDA}

The first was a simple LDA classifier as used for previous experiments. A majority voting post-processing was applied to the classification stream with a window length of 7, which was found to be the optimal trade-off between accuracy gain and controller delay in preliminary investigations. The proportional value for the identified class was computed as the average RMS value of all EMG signals, scaled to the MLVC of each motion. This method was used for reference to a standard pattern recognition method.

\section{Extended mode switching (eMSW)}

The second method for comparison was a straightforward extension of the commonly used mode switching method. As introduced in Chapter 1, in commercial prostheses, two electrodes are placed on independently controllable muscle regions of the forearm. This allows for the direct control of 1 DOF (two movements). In order to allow control over a second DOF, a co-contraction of both muscles groups is used as switching signal. In this study, this scheme was extended to the control of 3.5 $\mathrm{DOF}$ in form of a state machine. Co-contractions were used to cyclically switch from $\mathrm{WS} / \mathrm{WP} \rightarrow \mathrm{WF} / \mathrm{WE} \rightarrow \mathrm{KG} / \mathrm{HO} \rightarrow \mathrm{FP} / \mathrm{HO}$ and back to WS/WP (since $\mathrm{HO}$ was present twice, this system is referred to as $3.5 \mathrm{DOF}$ rather than $4 \mathrm{DOF}$ ). A visualization of this simple state machine is provided in Figure 4.3. 


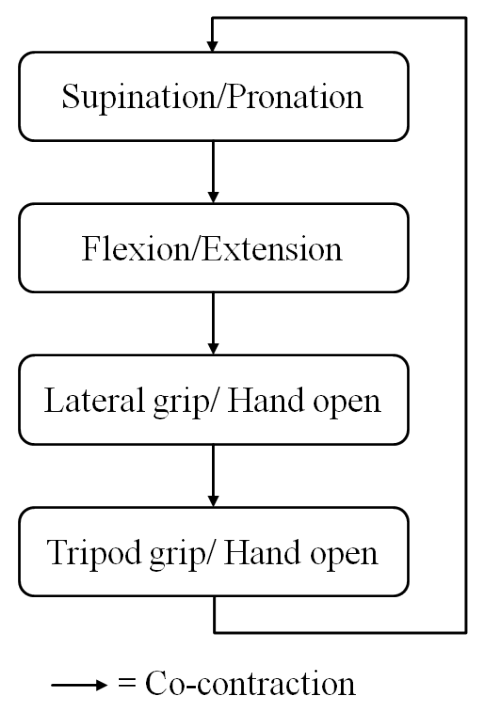

Figure 4.3: State machine for eMSW method. Arrows indicate transitions from one state to the next by co-contraction. A maximum of 3 switches is needed to reach any desired state, with the fourth co-contraction, the initial state is returned to. Reused and modified with permission [84] (C)2014 IEEE.

This extended mode switching method will be referred to as eMSW for the remainder of this chapter. It was included as a reference system, since it has not been shown yet in literature if this simple extension of the well accepted control for 2 DOF was also suitable for the control of 3.5 or 4 DOF. The switching diagram shown in Figure 4.3 was printed out and available for the subjects throughout all tests. Successful co-contractions were accompanied by a beeping sound for feedback.

The eMSW method was implemented with support from Otto Bock Healthcare Products GmbH, Vienna, Austria, which provided detailed information on how a cocontraction based switching system is realized in commercial prostheses (thresholds, timing, winning signal strategies...). While these confidential data will not be disclosed in this thesis, the adherence to these guidelines during the implementation of the software ensured similar performance as found in commercial systems. The eMSW method was tested only with the able-bodied subjects. With amputees it was preferred to record data with their own, commercially available prostheses as the reference scenario. It represents the current clinical state of the art (SOA). Investigating eMSW in addition to the SOA was not feasible due to time and fatigue constraints of the amputee subjects.

The sequence of testing the machine learning methods was randomized and subjects were blind to the chosen order. The eMSW control required a completely different control strategy and thus the subject had to be informed when this method was used. 


\subsubsection{Subjects}

In total, 14 subjects were recruited to participate in this study, split in a control able-bodied (10) and an amputee (4) subject group. All subjects were introduced to the design and goals of the study. Prior to their participation in the experiments, they signed an informed consent in compliance with the study approval of the local ethics committee.

Among the able-bodied subjects (age $30 \pm 4.7$ years), 3 female and 7 male subjects volunteered, all dominant right handed. The available hardware for this study did not allow mounting the able-bodied adapter for wearing the prosthesis and the EMG on the same arm. Therefore, the prosthesis (a left hand) was mounted on the left arm, while the EMG signals were recorded from the right arm. This setup was found to be intuitive after a very short familiarization phase (less than a few minutes) and proved to be beneficial because it distributed the physical strains (sustaining the weight of the prosthesis and performing the wrist and hand gestures) to both arms, which reduced fatigue.

The details on the amputees are summarized in Table 4.2. As mentioned previously, a customized socket to which the prosthesis got attached was manufactured for each amputee. The handling of the test prosthesis was therefore very close to how the wearers usually use their prostheses, which maximized the clinical relevance of this study.

\subsubsection{Applied test scenarios}

In order to estimate the potential of the three investigated prosthetic control schemes for application in prosthetic control, in this study the focus was put on online evaluations with the control of physical hand prostheses worn by the users. For able-bodied subjects, a splint was manufactured to attach the prosthesis to the sound forearm of a participant. For amputees, the same sockets as already fabricated for the study of Chapter 3 were reused, this time with the prosthesis attached to the socket. The test setup was thus very close to a realistic scenario of use.

With the prostheses attached to their forearms as shown in Figure 4.4, each subject was asked to complete the following tests in the same order as described in the following. This order was chosen so that the tests would increase in difficulty from simple to complex. 

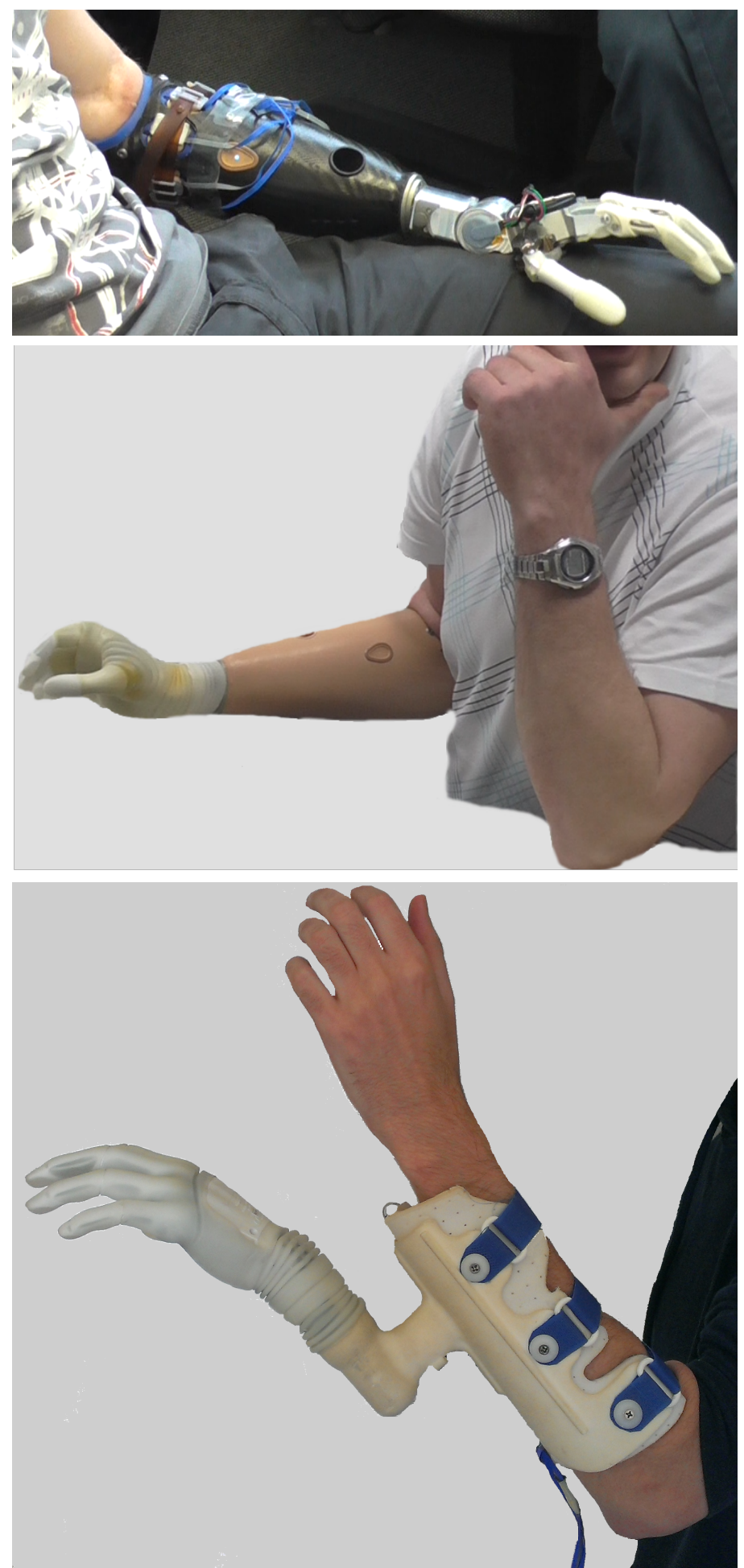

Figure 4.4: Prosthesis mounted on subjects. Upper two panels: one right (Amp1) and one left (Amp2) hand amputee, lowest panel: adapter for able-bodied subjects to wear the prosthesis for the tests. Reused and modified with permission [84] (C)2014 IEEE. 


\section{Box and Blocks test}

Originally, the box and blocks test was proposed as an assessment test for gross hand function of patients with cerebral palsy $[102,103]$, however due to its simplicity and versatility it can be used for grasping tests in general. Moreover, it is well studied and normative data for healthy adults [103] and minors [104] exist for reference.

The test consisted of two adjacent boxes of roughly $25 \mathrm{~cm}$ edge length and $7.5 \mathrm{~cm}$ height, separated by a $15 \mathrm{~cm}$ tall dividing barrier (for exact measures see [103]). One box was filled with cubes of $2.5 \mathrm{~cm}$ edge length and the test consisted in relocating as many cubes from the full to the empty box in $60 \mathrm{~s}$, one block at a time (Figure 4.5(a)). The test was repeated 3 times and the average number of transferred blocks was reported. Since this test only required opening and closing of the hand it was considered relatively easy from a control point of view.

\section{Clothes pin relocation test}

The clothes pin relocation test was proposed by Kuiken et al. [105]. Like the box and blocks test, it is simple to reproduce and has found good acceptance for quantifying upper limb function. The task which had to be performed by the subjects was to pick up a clothes pin clipped to a horizontal rod, rotate it by 90 degrees and place it on a vertical rod. The time for completing this maneuver 3 times in a row was measured and again the average of three repetitions was reported. In this study, the Rolyan ${ }^{\circledR}$ Graded Pinch Exerciser [106] was used, which is a standardized, commercially available version of that test. For the successful completion of this test, hand open/close and rotation functions of the transradial prostheses were required. Hence in this study it represented a test of medium difficulty.

\section{Block turn test}

After thorough literature research, to the best of the author's knowledge no standardized test was available which enforced the utilization of all movements (3.5 DOF) available in the hand prosthesis used for this study. The ULPOM (Upper Limb Prosthetic Outcome Measures) group gathered the most comprehensive list of tests [107], but none of them appeared concise and suitable enough for the control of a multifunctional hand prosthesis with the particular actuated DOF as utilized in this study. Therefore, a novel test was introduced for this study, as described in the following. 


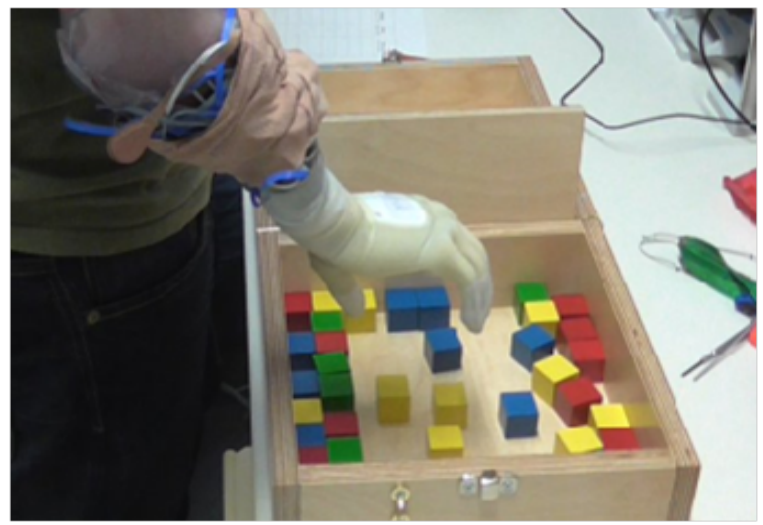

(a) A subject performing the box and blocks test. As many cubes as possible have to be transferred from the one box to the other over the barrier between them.

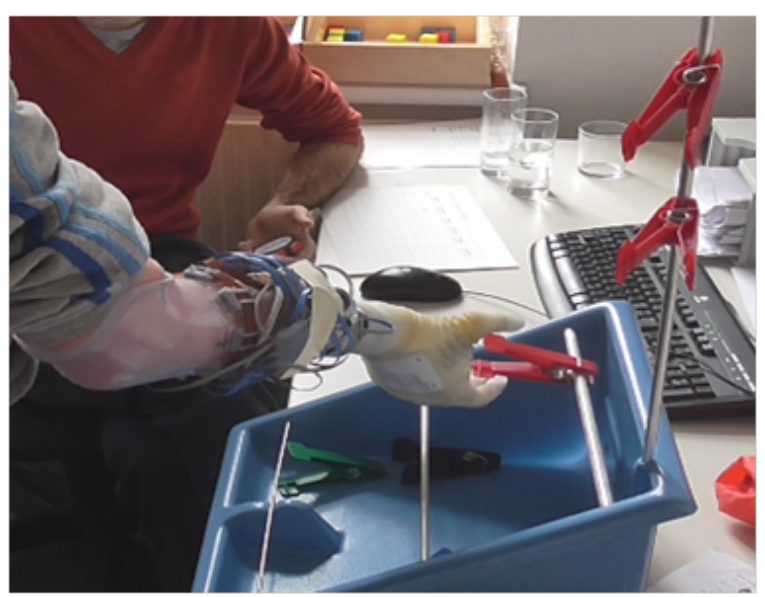

(b) A subject performing the clothes pin test. The task was to relocate the clothes pin from the lower, horizontal bar to the upper, vertical bar.

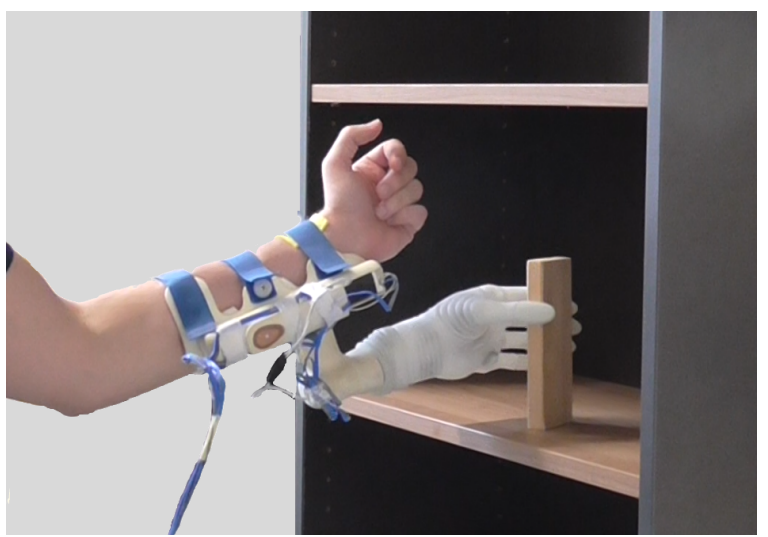

(c) An able-bodied subject in the middle of performing the block turn test, placing the block in the medium height shelf

Figure 4.5: Scenes of subjects performing the three practical tests applied in this study. 
The exercise of this test was to pick up a wooden rectangular shaped block with the dimensions of $15.8 \mathrm{~cm} \times 5.7 \mathrm{~cm} \times 1.7 \mathrm{~cm}$ lying flat on a shelf at shoulder level, rotate it, and place it on its short thin side like a book in a shelf at waist level. Then it was to be grabbed again and turned back down to its initial orientation. The movements required in each of the stages are detailed in Table 4.1 and depicted in Figure 4.6. This sequence of movements was to be performed as fast as possible and the required time was measured. If the block was dropped during the execution of the test it was repeated from the start. Only the times of fully successful trials were taken into account. As an additional measure to the speed, the number drops of the block was evaluated. The average time of three successful trials and the number of drops until three trials were successfully completed were evaluated. Less drops and faster completion times indicated a better control over the prosthesis.

Since this test required grasping in an elevated arm position and the control over all DOF provided by the prosthesis was necessary, this test was considered the most difficult of this study.

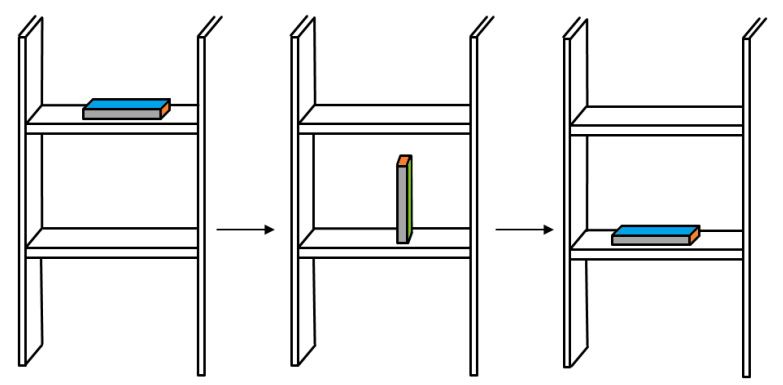

Figure 4.6: Schematic sequence of performing the block turn test. For closer description, see Table 4.1. Reused and modified with permission [84] (C)2014 IEEE.

Table 4.1: Sequence and required movements for performing the block turn test. Reused and modified with permission [84] (C)2014 IEEE.

\begin{tabular}{lc}
\hline Stage & Required movements \\
\hline Pick up block from shoulder level shelf & WF, FP \\
Rotate block upright & WS, WE \\
Place block on its small side like book in shelf at waist level & HO \\
Take the block again like a book or CD case & WP, KG \\
Rotate the block back to its original orientation and release it & WP, HO \\
\hline
\end{tabular}


Table 4.2: Details on amputee subjects. CoCo is used as short-hand for co-contraction, PRE for pattern recognition experience. Reused and modified with permission [84] (c)2014 IEEE.

\begin{tabular}{ccccccc} 
Subject & Age & Amputee & Stump length & Everyday prosthesis (movements) & Control method & PRE \\
\hline Amp1 & 26 & 3 years & medium & Michelangelo + rotation $(5)$ & CoCo + 4-channel control & $>60$ hrs \\
Amp2 & 35 & 7 years & medium & Sensorhand speed + rotation $(4)$ & 4 independent signals & $\sim 50$ hrs \\
Amp3 & 27 & 1 year & short & Sensorhand speed + rotation $(4)$ & 4-channel control & $\sim 20$ hrs \\
Amp4 & 29 & 3 years & medium & Michelangelo $(3)$ & CoCo & $\sim 25$ hrs
\end{tabular}

\subsubsection{Questionnaire}

Since the tests presented above solely relied on timed results, an additional, subjective measure of user confidence in the control methods was assessed in form of a short questionnaire with the questions as described in the following list.

1. Which method did you think resulted in unintentional movements more often?

2. Which method gave you the feeling of better control over the prosthetic movements?

3. With which method did you have finer proportional control?

4. With which method do you think you were faster on average?

5. Which method did you prefer overall and would like to see in your own prosthesis?

These questions were asked to the amputee subjects only since they were regarded as the more relevant group for this subjective rating.

\subsubsection{Hardware control system}

The prosthesis used for the experimental sessions was a commercially available Michelangelo hand from Otto Bock HealthCare Products GmbH, Vienna, Austria. It was attached to prototypes of actuated wrist rotation and flexion/extension units by the same manufacturer. The prosthesis thus allowed control over 3.5 DOF. As for the data recording as described in Chapter 2, eight 13E200 $=50$-AC electrodes were placed equidistantly around the circumference of the subjects' forearms. For able-bodied subjects, a custom manufactured mounting system was employed to hold the electrodes in place and for amputees their sockets were used. The signals were conditioned by the active electrodes and A/D converted by the Axon bus master (Otto Bock) at 10 bit 
resolution with a sampling frequency of $1 \mathrm{kHz}$. The signals were transferred to a PC (Intel i7, $1.73 \mathrm{GHz}, 6 \mathrm{~GB}$ memory, Microsoft Windows 7) via Bluetooth connection, where the signal processing steps for extracting the user intent with the algorithms described above were implemented in a custom C\# software package. The resulting prosthetic movement commands were then sent back to the prosthesis again via the same Bluetooth link to control it in near-realtime. The delay of the Bluetooth connection (from sending the movement command to measuring a movement with the hand internal sensors) was quantified in previous experiments to be in the range of $<50-400 \mathrm{~ms}$. This delay was noticeable but acceptable for the tasks completed in this experiment.

\subsubsection{Data acquisition}

In order to acquire the training data for the LDA and CSP-PE methods, the same software and procedures as described in Chapter 2 were used, but only 3 runs were recorded per subject. The modified Hudgins feature set (RMS, ZC, SSC, WL) was extracted from the signals. With able-bodied subjects, the data were recorded in one relaxed arm position (upper arm hanging, elbow $90^{\circ}$ flexed, forearm pointing forward) and took around 15 minutes including breaks. All 7 movements the prosthesis was capable of performing were included plus NM. With amputees, 3 different arm positions (relaxed hanging, stretched to head level and pointing forward, [67]) were included, with the prosthesis mounted. For Amp1 and Amp2 the same classes as for the able-bodied subjects were used. For Amp3 and Amp4, WF and WE were excluded, since these subjects were not able to perform these movements consistently and distinguishably from all other movements. To avoid fatigue, substantially more breaks were granted to the amputees, doubling the recording time.

\subsubsection{Statistical analysis}

In order to evaluate whether the used control methods resulted in significantly different task completion measures, an ANOVA for repeated measures with one factor of three levels (CSP-PE, LDA, eMSW) was conducted. Subjects were treated as random factors. When the ANOVA showed a significant difference, a Tukey-Kramer post hoc comparison $[89,90]$ was applied to investigate pair-wise differences between methods. The analysis was only carried out for able-bodied subjects. For amputees, the results 
were quite variable and due to the low number $(n=4)$, the individual results are presented rather than summarizing statistics.

\section{$4.2 \quad$ Results}

\subsubsection{Able-bodied subjects}

In preliminary tests, all experiments were conducted within one session, after which subjects reported substantial fatigue in the left arm (the one carrying the prosthesis). Therefore, for the final assessments of this study, the experiments were split in two days: On the first day, subjects performed all tests with the machine learning based control methods (LDA, CSP-PE) and on the second day, eMSW was investigated. With this setup all subjects were able to complete the experiments reporting at most mild fatigue, with the exception of one female subject, who still reported strong fatigue, accompanied by mild shoulder pain and substantially decreased performance was apparent, especially with LDA. For this subject the experiments were thus aborted and the data were excluded from all further evaluations.

In Figure 4.7 exemplary estimation results of LDA with no post-processing, LDA with majority voting of 7 and CSP-PE are shown for the same EMG data. The graph shows that in the center of the investigated time segment, the first EMG channel was affected by noise, resulting in mis-classifications of LDA, which could also not be removed with a majority vote of 7 , as used in this study. In fact, a majority vote of 32 was necessary to remove all shown mis-classifications, but this would also have introduced one second delay in the real-time control. CSP-PE however was capable of suppressing wrong movements in this scenario.

The statistical analysis revealed that the control method had significant influence in all tests $(p<0.01$ for all). Therefore, in the following only the pairwise differences will be presented per test.

\section{Results of box and blocks tests}

Average times for picking up, transferring and releasing one cube from one box to the other were $3.1 \pm 0.4 s, 4.1 \pm 0.9 s$ and $2.7 \pm 0.5 s$ for CSP-PE, LDA and eMSW respec- 

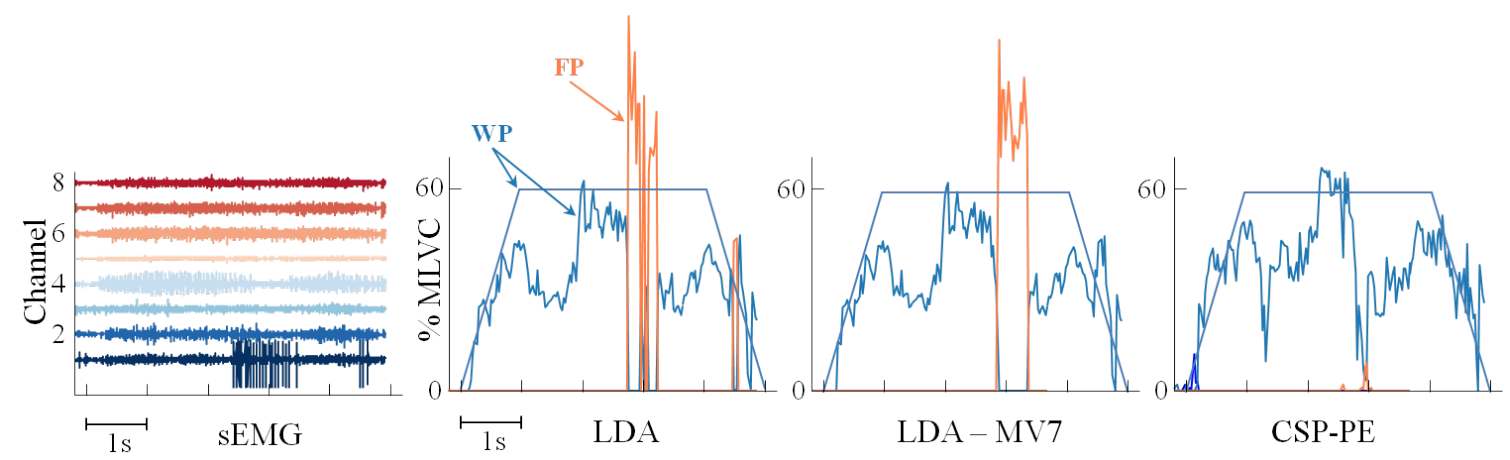

Figure 4.7: Exemplary estimation results for the same EMG data by LDA, LDA with a majority vote of 7 and CSP-PE. It is apparent that approximately in the center of the displayed sequence the first EMG channel was affected by noise (likely due to electrode lift-off). In this case, LDA output some wrong estimations, which were also not removed by majority voting. The subject was asked to perform WP, but erroneous FP activations occurred. CSP-PE activation was strongly decreased for this period of time, minimizing the risk for performing a wrong movement. Reused and modified with permission [84] (C)2014 IEEE.

tively $^{1}$. In this comparison, LDA was significantly worse than both other methods ( $p \leq 10^{-3}$ for both). CSP-PE was also outperformed by eMSW, but this difference was not statistically significant $(p=0.19)$. The good performance of eMSW can be explained by the fact that for this test no mode switching was necessary (only open/close required) and thus eMSW allowed for fast grasping and releasing. The only times the users were interrupted in their flow of grasping was when an erroneous co-contraction was detected (mostly because the subjects became too confident and acted too fast, before their muscles were relaxed), but this occurred only on rare occasions. The results per method in the box and blocks test are summarized in Figure $4.8(\mathrm{a})$.

\section{Results of clothes pin tests}

This test required control over at least 2 DOF (rotation and hand open/close). Therefore, the eMSW method was expected to perform worse than the machine learning methods, which allowed direct accessibility to each function. Indeed, the average time required to complete this test across subjects was $59.3 \pm 18.5 \mathrm{~s}$, which was significantly slower than with $\operatorname{LDA}(31.6 \pm 14.1 s)$ and CSP-PE $\left(25.6 \pm 7.3 s, p<10^{-3}\right.$ for both). The better performance of CSP-PE compared to LDA was not significant

\footnotetext{
${ }^{1}$ In this study, the average times to transfer one block are reported, which is in contrast to most other papers where the number of blocks transferred in $60 \mathrm{~s}$ was reported. This choice was made so that for all evaluation metrics used in this paper "less is better", allowing for more intuitive, direct comparison of the results achieved per method and test. A conversion to blocks in $60 \mathrm{~s}$ for comparisons is trivial.
} 
$(p=0.47)$, however the completion times were much more consistent in CSP-PE than in LDA (compare 14.1 vs. $7.3 \mathrm{~s}$ standard deviation). The average completion times per method in the clothes pin test are summarized in Figure 4.8(b).

\section{Results of block turn tests}

The block turn test proved to be the most selective one, revealing significant differences among all methods ( $p<10^{-3}$ for all comparisons). The best performing algorithm was CSP-PE with an average task completion time of $25.5 \pm 6.2 \mathrm{~s}$, followed by LDA $(34.1 \pm 12.5 s)$ and eMSW $(48.6 \pm 10.0 s)$. In the additional measure evaluated for the block turn test, the number of drops of the block, eMSW and CSP-PE were not significantly different from each other $(p=0.27)$ but both where significantly better than LDA $\left(p<10^{-3}\right)$.

Thus, eMSW again proved to be slow in tasks where several DOF were to be controlled. Additionally, subjects reported that they found this control scheme confusing and much more complicated than the machine learning based methods. The results of CSP-PE were the best with statistical significance, and also with the lowest variance. This objective result is in good correspondence with the subject's feedback, who reported mostly that CSP-PE felt better controllable than LDA (although no detailed interrogation was performed with these subjects). The completion times and number of drops separated by method for the block turn test are summarized in Figure 4.8(c) and Figure 4.8(d).

\subsubsection{Amputee subjects}

The entire experiment was completed by all amputee subjects, with the exception of Amp4 due to sickness on the last day of the study, thus for this subject no comparative data with his own, commercial prosthesis could be acquired. Moreover, since this subject only had a Michelangelo hand without rotation or wrist flexion unit, evaluating the clothes pin test and block turn test would have been difficult for this subject. Since the other 3 subjects had all different hands and wrist units (see Table 4.2), the block turn test had to be simplified accordingly (starting position of the block at waist level, no KG for the second part of the turn), which made a comparison to able-bodied and also among the amputee subjects difficult. Therefore, no direct comparisons were made and the results of the amputees with their SOA prostheses are reported in the end of this section for reference. 


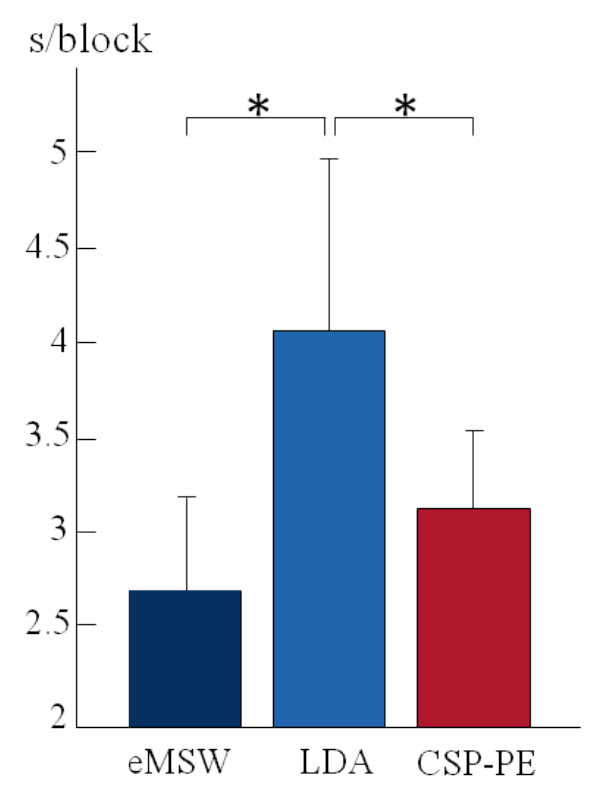

(a) Box and blocks

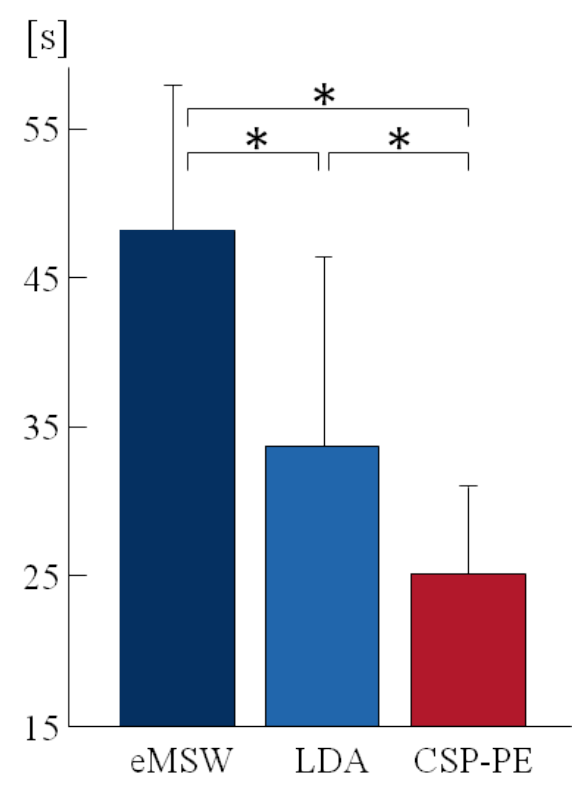

(c) Block turn - Time

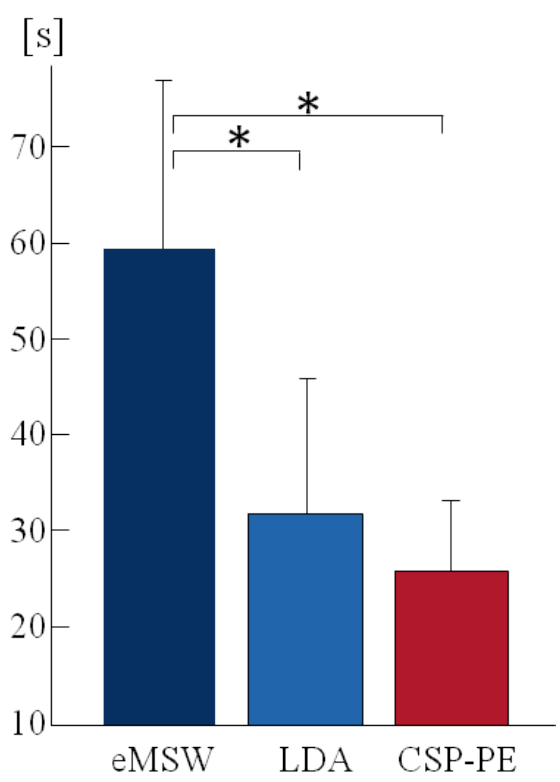

(b) Clothes pin

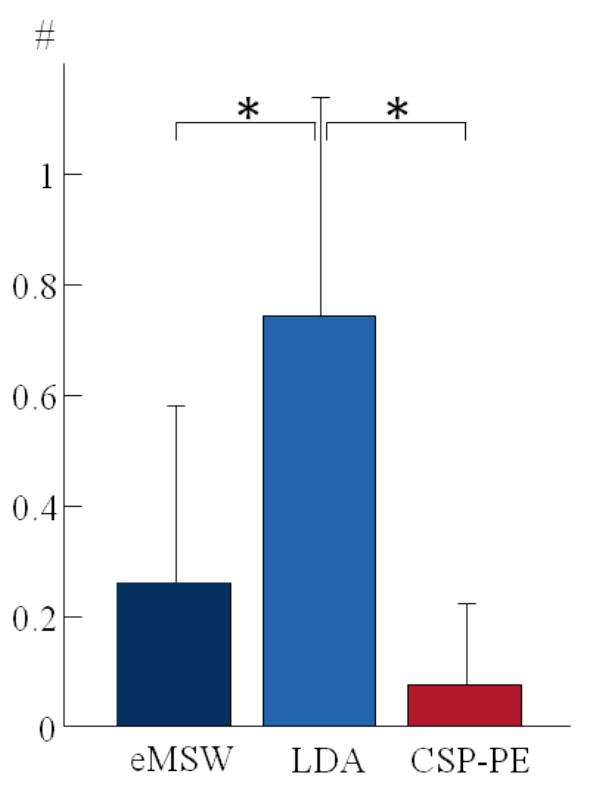

(d) Block turn - Drops

Figure 4.8: Results of the three applied tests conducted in this study achieved by able-bodied subjects, grouped per test. eMSW performed well in the simple box and block test but proved unsuitable for more complex tasks. CSP-PE was not significantly slower than eMSW in the box and blocks test and outperformed both other methods in the more complex tasks. Reused and modified with permission [84] (C)2014 IEEE. 


\section{Results box and blocks test}

Amputee subjects were faster using CSP-PE (2.45 s) compared to LDA (2.74 s) for transferring the cubes in the box and blocks test, resulting in an increased transfer rate of 22.1 to 25.1 blocks in $60 \mathrm{~s}$. The individual improvements were variable with 4.3, 5, 0.3 and 2.3 (Amp1-Amp4) blocks relocated more in $60 \mathrm{~s}$ with CSP-PE than with LDA, but the advantage of CSP-PE over LDA was consistent in all 4 amputees. The results per method in the box and blocks test are summarized in Figure 4.9(a).

\section{Results clothes pin test}

The average difference between LDA and CSP-PE for completing the clothes pin test was only minor $(22.75 \mathrm{~s}$ vs. $22.25 \mathrm{~s})$. However, reporting the average in this case is not very representative, since the results varied substantially among subjects: While Amp1, Amp2 and Amp4 completed the task on average $2.7 \mathrm{~s}, 0.7 \mathrm{~s}$ and $4.3 \mathrm{~s}$ faster with CSP-PE than with LDA, Amp3 required 5.7s more time. When asked for his feedback, interestingly this subject preferred the control with CSP-PE because he had more confidence in that control. With LDA he perceived more mis-classifications and he thus did the task in a faster but also sloppier way: For example, releasing a clothes pin on the vertical rod was not only possible by opening the hand but also by rotating the wrist until the grip of the prosthesis lost the clothes pin and it snapped back to the rod. While not elegant, this was not forbidden in the test and sometimes faster for the subject if the control of hand open was poor or unreliable. The average completion times per method in the clothes pin test are summarized in Figure 4.9(b).

\section{Results block turn test}

The average time needed for the amputee subjects to complete the block turn test with CSP-PE was $22.75 \mathrm{~s}$ and 25.3 s with LDA. Amp1, Amp2 and Amp4 required 9s, $3.3 \mathrm{~s}$ and $2.7 \mathrm{~s}$ less time with CSP-PE, whereas Amp2 was faster by $2.8 \mathrm{~s}$ with LDA. This subject experienced problems with CSP-PE when grasping the block from the top shelf. The wooden block was only once dropped (by Amp2) with CSP-PE and three times with LDA (once each by Amp1, Amp2 and Amp4). The completion times and number of drops separated by method for the block turn test are summarized in Figure 4.9(c) and Figure 4.9(d). 
s/block

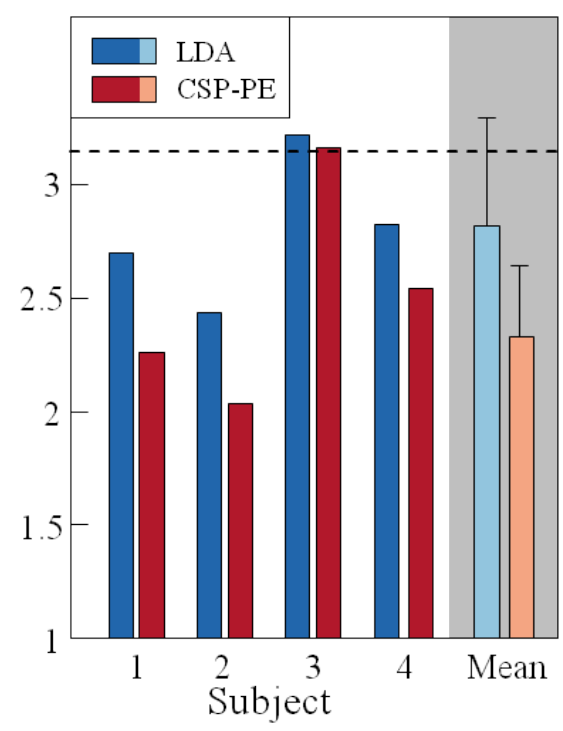

(a) Box and blocks

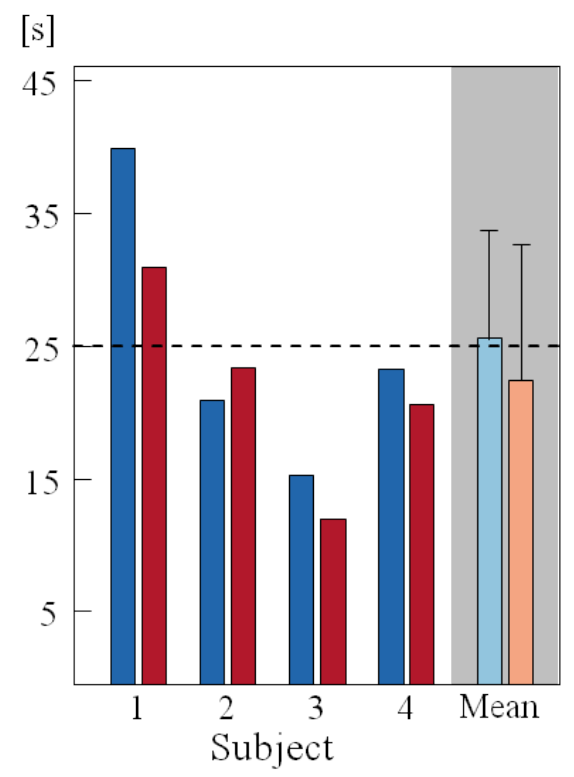

(c) Block turn - Time [s]

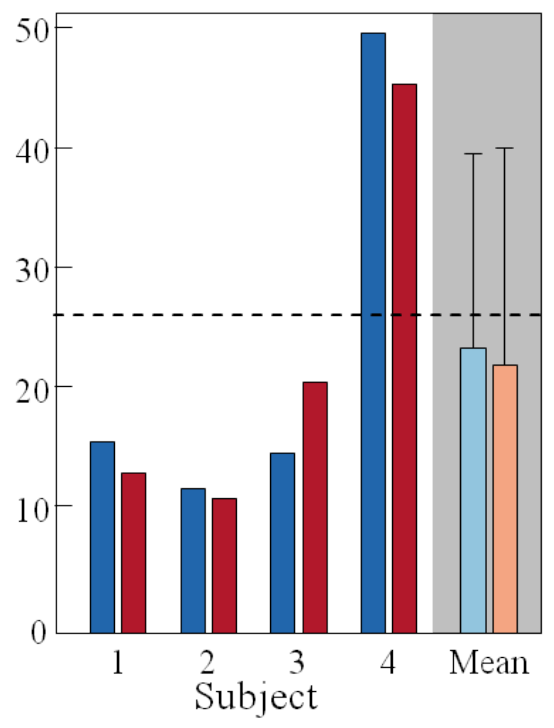

(b) Clothes pin

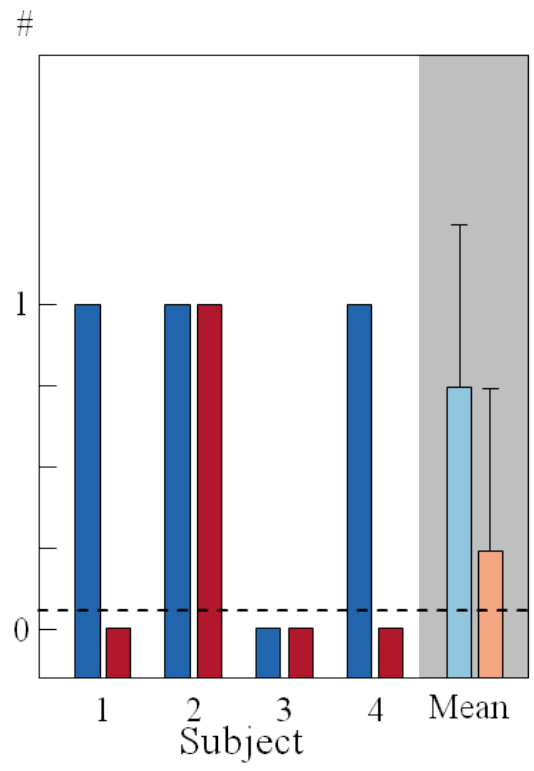

(d) Block turn - Drops

Figure 4.9: Results of the three applied tests of this study achieved by amputee subjects. The last panel shows the average over the subjects. In 10 out of the 12 timed measures (4 subjects, 3 tests) CSP-PE outperformed LDA and it also performed better on average in the number of block drops. The dashed horizontal lines represent the average results for able-bodied subjects achieved with CSP-PE for reference. Reused and modified with permission [84] (C)2014 IEEE. 
Table 4.3: Amputee subjective method ratings as answers to the questions introduced in Section 4.1.5 (N.D. denotes no difference perceived). Reused and modified with permission [84] (C)2014 IEEE.

\begin{tabular}{lcccc}
\hline Question & Amp1 & Amp2 & Amp3 & Amp4 \\
\hline More unintentional movements & LDA & N.D. & LDA & LDA \\
Better controllability & CSP-PE & N.D. & CSP-PE & CSP-PE \\
Finer proportional control & CSP-PE & CSP-PE & CSP-PE & CSP-PE \\
Subjectively faster task completions & CSP-PE & N.D. & CSP-PE & N.D. \\
Overall preferred method & CSP-PE & CSP-PE & CSP-PE & CSP-PE \\
\hline
\end{tabular}

\section{SOA prostheses tests}

In addition to the experimental test prostheses, three of the amputee subjects also completed the tests with their own SOA prostheses. Amp1 performed considerably better with his own prosthesis in the box and blocks test than with CSP-PE (41.7 blocks, compared to 27) and also in the clothes pin test (11s vs. $13 \mathrm{~s}$ on average). He completed the simplified block turn test in 15.7 s. Amp2 moved one block less per $60 \mathrm{~s}$ in the box and blocks test compared to CSP-PE and was almost equally fast with his SOA prosthesis in the clothes pin test (10.7 s SOA, $11.0 \mathrm{~s}$ CSP-PE). He needed $7.3 \mathrm{~s}$ for the simplified block turn test (no WF/WE, no KG). Amp3 moved 3 blocks less with his prosthesis compared to CSP-PE and was $8.7 \mathrm{~s}$ slower in the clothes spin test. He completed the block turn test in $14.7 \mathrm{~s}$ with the same simplifications made as for Amp2.

In summary, the performance of the amputees with their own prostheses was dependent on the level of training they had with it, however for all subjects this was more than one year. This is compared to only a few hours of familiarization with the methods investigated in this study.

After completing the tests, all amputee subjects were asked 5 predefined questions (see Section 4.1.5) to evaluate their subjective feedback on the two machine learning methods. The two methods were referred to as methods $A$ and $B$ throughout the sessions in order to keep the subjects blinded towards the methods and were only revealed to them after answering the questions. In general, subjects preferred CSP-PE over LDA. The results are summarized in Table 4.3. 


\subsection{Study discussion}

Following the motivations of the previous chapters, further efforts for improved robustness in myoelectric prosthetic control of multifunctional prostheses were pursued in this study. The work of the previous studies was extended here to a novel proportional regression method which incorporated an inherent mechanism for suppressing potentially wrong movements.

\subsubsection{Machine learning methods}

In Figure 4.2 an exemplary result of the estimation achieved by CSP-PE when applied to sEMG signals was shown and in Figure 4.7 how CSP-PE was able to correct for noisy signals in comparison to LDA. The promising results of these exemplary demonstrations were quantitatively confirmed in a study with 10 able-bodied and 4 amputee subjects in a realistic test setup. The control of a physical prosthesis in real-time for the completion of three different applied tests of varying difficulties represented a clinically highly relevant test scenario.

The obtained results showed that CSP-PE consistently outperformed LDA in almost all tests in both subject groups. In healthy subjects, it yielded the best results in the 2 most complex of the 3 tasks. It was only outperformed slightly by eMSW in the box and blocks test, since the extended conventional control scheme allowed for very fast open-close cycles without needing to switch between movements. In amputees, CSP-PE achieved the best results in 10 out of 12 experiments ( 4 amputees, 3 tests). With this consistency of results it is concluded that CSP-PE was the best performing method investigated in this study.

The good performance of CSP-PE can be attributed to its mathematical properties of maximal class separation and suppression of wrong movements based on the likelihood for correct estimations. Similar results were reported in [52] in an abstract avatar control on a computer screen and follow the same rational as described in Chapter 3 and [84]. Thus, the benefit of canceling wrong movements was already anticipated but shown for the first time in this study for a physically controlled prosthesis. In addition to its self correcting properties, the proportional control output for each movement was found to be very beneficial and allowed fine controlled maneuvers. 


\subsubsection{Extended state-of-the-art control}

In order to support the need for direct control of multi-DOF hand prostheses, the eMSW method was included in the investigated test protocol. As expected, the method was very unintuitive and cumbersome for the naïve users to operate. They often got lost in the control state machine, despite receiving auditory feedback for each successful co-contraction and the flow chart of Figure 4.3 being visible for the subjects at all times for support. Moreover, the eMSW experiments were conducted last and on a separate day, so the subjects already had the most experience with the prosthesis and the tests and were well rested. In the clothes pin and block turn test, control with CSP-PE was approximately twice as fast compared to the eMSW method and also LDA outperformed eMSW significantly. Together with the negative impressions reported by the subjects it is concluded that eMSW was not suitable for the control of the 3.5 DOF prosthetic hand used in this study. For hand opening and closing only however, as required in the box and blocks test, this method was found to perform very well, since in that case switching between DOF was not required. Certainly, several improvements to this naïve straightforward extension of the clinical SOA could be made, such as including codes (short or long co-contractions, automatic return to often used functions after inactivity, etc.) but the determination of such heuristic rules was out of the scope of this work. Also, when users have sufficient time to learn the control method, good control with the SOA switching paradigm can be achieved, as demonstrated by the amputees with their own prostheses (although they had a limited number of actuated DOF).

\subsubsection{Block turn test}

In addition to the proposed machine learning method, a novel test for assessing manual dexterity of upper limb prostheses was proposed in this study. In practice, the block turn test proved to be very selective and revealed many shortcomings of the evaluated control mechanisms. The test is easily replicable, adaptable and in theory could also be performed by upper arm amputees. The evaluation and interpretation of the test results is straightforward. In summary, this test may be a good candidate for becoming a standardized evaluation metric for prosthetic hand function assessment.

For the SOA tests with amputees, the block turn test had to be simplified signif- 
icantly, since none of the amputees had a SOA prosthesis with an actuated wrist flexion/extension unit and only one subject could perform two grip types. This also demonstrates the usefulness of these functions for specific tasks as described in this test, provided that a suitable control method is supplied.

\subsection{Study conclusion}

A novel proportional control algorithm for myoelectric multifunctional hand prostheses was introduced and evaluated in this study. The method was evaluated with able-bodied and amputee subjects and compared to two other algorithms: one academic state of the art and one extended version of a commercially available method. The evaluation setup was chosen very realistically, with the real-time control of a physical prosthesis in three different tests of pick and place actions of varying difficulty, among which one newly proposed test.

The introduced control method outperformed the other methods in the majority of all tests investigated. The achieved results were consistent between the able-bodied and amputee subject groups. The block turn test demonstrated selectivity, adaptability and reproducibility. In summary, the demanding test setup and the consistently good results achieved with CSP-PE indicate a significant advancement in the efforts made towards reliable, robust and intuitive control of multifunctional hand prostheses. 


\section{$5 \quad$ Combining sequential and simultaneous regressors}

Natural control of sound limbs is characterized by combined and parallel movements strictly sequential movements are perceived as robotic and inhuman. Imagine for example the sequence of taking a glass of water in front of someone: One would rotate and extend the wrist to position the hand, open the fingers and extend elbow and shoulder - all at the same time, in order to grasp the glass efficiently and in the correct orientation to avoid spilling. Broken down in its individual components, this every-day movement suddenly appears indeed very complex - especially when one is faced with the task of moving an artificial limb as natural in appearance as possible. In a study dedicated to reveal frequent reasons of upper limb prosthesis device abandonment [14], it was reported that "Dissatisfaction with prosthetic technology" and "Appearance of the prosthesis" were among the most critical factors. It is therefore not astonishing that up to $50 \%$ of all myoelectric prostheses are reported to be never used by their owners $[14,108,109]$. Providing prostheses, which are intuitive to use and appear more natural in their functioning and moving, may thus be desired by many users $[6,109]$.

To facilitate the intuitive control of multifunctional prostheses, pattern recognition of EMG signals has been investigated for many decades. Recently, research efforts have started to focus on the proportional simultaneous estimation of movement across several DOF (for references see the introduction chapter of this thesis, Section 1.5.2). The developed regression methods map the exerted force estimated from the EMG to the activities of all joints. They allow the simultaneous estimation of activation of several DOF, and each activation estimate is independent in its amplitude from the rest. Therefore, regression methods allow for very life-like movements. One drawback of these methods is however that they only allow for the control of a limited 
number of DOF - typically 2 [110]. Including more DOF results in considerably more erroneous activations of unintended movements, making dexterous control difficult. This is however, as shown in the previous studies, achievable with pure sequential estimators.

In summary, sequential control algorithms such as LDA and CSP-PE and simultaneous proportional regression models have disjoint strengths and weaknesses. Therefore, the combination of both types of control appears as a promising idea: In case of subtle, high precision single-DOF movements, a robust sequential controller should be used. For gross positioning and orientation of the prosthetic limb, simultaneous and proportional control is favorable for its natural appearance. This idea was pursued in the present study, in which a methodology for achieving the combination of "expert algorithms" for different situations is proposed and evaluated. The most critical step of this approach is to decide whether a movement should be estimated in a simultaneous or sequential manner. Several solutions to this problem are investigated first.

\subsection{Combining estimators}

In this section a methodology is described which enables the combination two estimators with complementary advantages for sequential and simultaneous wrist and hand movements. The task would be relatively simple to accomplish if data of both singleDOF and multi-DOF movements were available for training. However, due to the exponential growth of combination possibilities with the number of controlled DOF, this would significantly increase the data recording time and is thus not desirable. Therefore, in a practical setup only training data of the single-DOF data are available which are required for training of the movement estimators. A schematic representation of the system combining sequential and simultaneous regression methods as used in this study is shown in Figure 5.1.

\subsubsection{Embedded dimensionality estimators}

The scientific techniques designed to solve binary recognition problems, where only data of one (positive) class are accessible for training and data of the counter (negative) population are not available, are called novelty detection or one class classi- 


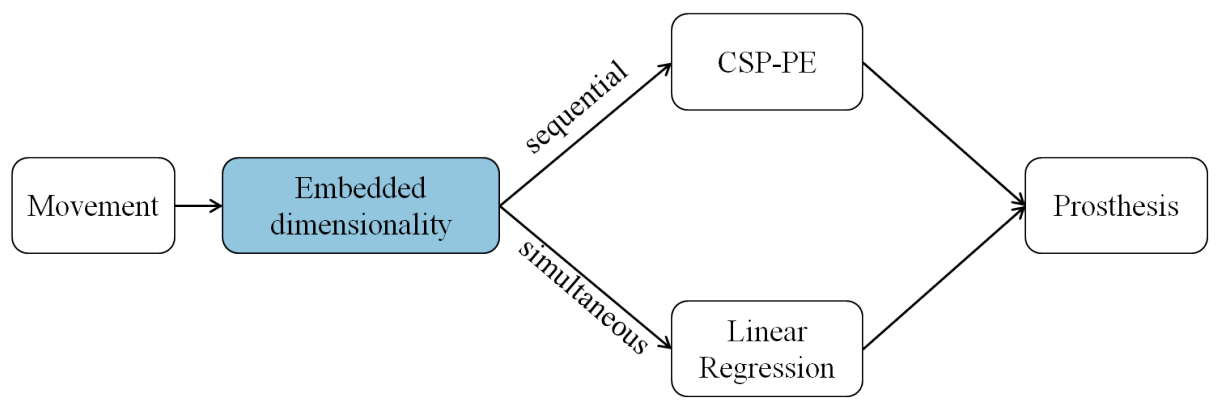

Figure 5.1: Schematic representation of the proposed system. For a feature vector originating from a given movement, first its embedded dimensionality is estimated. Depending on the result, the feature vector is forwarded to a specialist for sequential or simultaneous movements. The resulting estimate from that specialist is used for controlling the prosthesis.

fication [111] methods. Consider for example the design of a jet engine, in which a sensor based failure detection system should be integrated. Acquiring data from a normally running engine can easily be done during routine tests. However intentionally inducing a variety of damages in order to observe corresponding sensor responses is very costly in this scenario. Although sometimes less drastic, the same principle can be applied to many other problems, which gave rise to the research field of novelty detection. The same rational is also applicable to challenge in this study as described above, where patient time is very expensive. This allows only for the collection of data corresponding to different single-DOF movements. These form the data with the positive label. The unavailable data are those which stem from combined movements. During the online application phase, a method trained on the available data should be able to determine if the currently applied data stemmed from a single-DOF movement or not - and thus had to be of multi-DOF activation origin.

One well accepted method, which has even advanced to be regarded as the gold standard solution in novelty detection, is called one class support vector machine (OCSVM). It was proposed by Schölkopf et al. [112] and uses the kernel trick to map the data of the positive class to a high dimensional space such that they are compact and well separated from the origin in that space. That is, the smallest hypersphere in that space which encloses all training data is identified. For a newly applied sample it is evaluated whether it is inside or outside that hypersphere (for details see [112]). Apart from OCSVM, several other competitive methods have been described. Five methods were selected from literature as the most promising candidates, augmented by slight alterations and newly conceived ideas which resulted from the previous chapters, are described briefly in the following. Since they are used to es- 
timate the embedded dimensionality of the observed EMG (single-DOF or multi-DOF activations), these methods will be referred to as embedded dimensionality estimators (EDE) in the following.

\section{OCSVM}

The state-of-the-art novelty detection method, as described above. The implementation in Lib-SVM was used for the experiments [113].

\section{KNFST}

The Kernel Null Foley-Sammon Transform (KNFST) was proposed by Bodesheim et al. [114]. The Foley-Sammon method is identical to the Fisher transformation as described in Section 1.5.1 and is often referred to by that name in subspace learning. In KNFST, the standard Fisher criterion as in Equation (1.8) is optimized, however the within-scatter matrix of each class $\Sigma_{w}$ is reduced to 0 , which means that the data of that class have zero variance in the transformed space, i.e. they are projected to a single point. This is only achievable in a high-dimensional space, such as obtained by applying a kernel transformation [114]. For application, the novel feature vector is mapped to the high dimensional space and the minimum euclidean distance of the transformed point to any of the trained class points is taken as the measure for novelty. An empirically determined threshold to that distance gives the decision for novelty or not. The benefit of KNFST with respect to OCSVM is that it describes each trained base class individually, while OCSVM treats all training data as coming from the same class. In the present problem, multiple heterogeneous classes (training data per movement class) form one super class of single-DOF movements. It may therefore be beneficial to consider this a priori knowledge as done in KNFST. The major drawback of KNFST however is that it requires computation of the full kernel matrix with all training data, thus requiring considerable computation time during the application phase. The implementation as available in [115] was used for this study. Only every $4^{\text {th }}$ training point could be used, otherwise an OutOf MemoryException occurred (PC with Intel i7 core, Windows 764 bit, 6 GB RAM and Matlab 2012a). 


\section{MD}

A relatively simple technique is to calculate the minimum distance of a given point to any of the training classes similarly to KNFST, but directly in the input space and without the Fisher transformation [111]. As distance measure the Mahalanobis distance $(M D)$ is suitable, assuming Gaussian distribution of each class. The $M D$ of a feature vector $\boldsymbol{x}$ to class $i$ with the class mean vector $\boldsymbol{\mu}_{i}$ and covariance matrix $\Sigma_{i}$ is calculated as $[38,39]$ :

$$
M D=\left(\boldsymbol{x}-\boldsymbol{\mu}_{i}\right)^{T} \Sigma_{i}^{-1}\left(\boldsymbol{x}-\boldsymbol{\mu}_{i}\right)
$$

In contrast to the kernel based methods described above, this approach is computationally inexpensive and does not require hyperparameter optimization. However, a threshold for $M D$ above which a feature vector is classified as novel has to be determined. In this approach one threshold for all classes is chosen.

\section{MD-IND}

This minor variation is mostly identical to the MD approach described above, with the difference that a novelty threshold for each movement class (WS, WP, WF, WE, $\mathrm{HO}, \mathrm{KG}, \mathrm{FP}$ ) is determined individually.

\section{MD-LDA}

In another variation of the MD method, the LDA transformation was applied to the feature data before calculating the MD in the transformed space. This was included to investigate whether the Folley-Sammons transformation (=LDA) of KNFST was a critical step for successful novelty detection.

\section{$\mathrm{kNN}$}

Rather than assuming an underlying Gaussian distribution of the class data and fitting the corresponding parameters as done with the MD based approaches, the non-parametric $\mathrm{kNN}$ approach was proposed for novelty detection [111]. The approach is almost identical to MD, but rather than evaluating the minimal MD of the feature vector to all classes, the minimal euclidean distance to any set of $k$ neighbors was considered. Again, a threshold based novelty detection rule was applied. 
The parameter $k$ was set to 5 . In a straightforward implementation, this approach is very slow, since computation time increases with the number of training data (time complexity $O(N d)$ for $N$ training points of dimensionality $d$ ).

\section{CSP-PELL}

A further measure for recognition of known data can be extracted from the CSP-PE estimator introduced in Chapter 4. As part of the computation, the likelihood (CSPPELL) of each estimation (expressed as the maximum of the contrasts normalized to sum 1) is obtained. This can directly be used as the estimate for novelty. Again, a simple threshold between 0 and 1 has to be determined.

\section{LDA-LL}

Similar to CSP-PELL, the classification likelihood of an LDA classifier trained on the single-DOF data can be used.

\subsubsection{Methods for identifying the optimal EDE}

For a preliminary investigation to determine the most suitable of the EDE methods described above, an offline evaluation was performed preceding the online experiments described later in this chapter. For this purpose, 6 able-bodied subjects were recruited and sEMG data corresponding to 7 active single-DOF movements (WS, WP, WF, WE, HO, KG, FP) plus a rest class as well as data from the 4 combined movements $\mathrm{WS}+\mathrm{WF}, \mathrm{WS}+\mathrm{WP}, \mathrm{WP}+\mathrm{WF}$ and $\mathrm{WP}+\mathrm{WE}$ were acquired. The training and recording paradigm was the same as used in Studies I, II and III, adapted to be also suitable for combined movements. In one run, all movements were performed 3 times (at 30\%,60\% and 90\% MLVC) and in total 3 runs were recorded. For the combined movements, subjects were asked to perform both of the partial movements equally at the prompted contraction level, as they would also like to use it in an online application. In an offline analysis, the $8 \mathrm{EDE}$ methods were trained with two runs of the single-DOF data only, and the withheld run as well as all 3 runs of the combined movements were tested (3-fold cross-validation). This was repeated until all runs were withheld once from training and served as test data. The novelty detectors acted as EDEs by applying thresholds as described above, which resulted in a two class classification for each presented feature vector: single-DOF or combined-DOF 
5.1. Combining estimators

Table 5.1: Elapsed CPU times in [s] per EDE method to estimate all data of one condition

\begin{tabular}{lcccccccc}
\hline & OCSVM & KNFST & MD & MD-IND & MDLDA & kNN & CSP-PELL & LDA-LL \\
\hline Single-DOF & 0.02 & 2.1 & 0.02 & 0.02 & 0.01 & 126.9 & 0.66 & 0.02 \\
Combined-DOF & 0.04 & 4.7 & 0.03 & 0.03 & 0.01 & 191.1 & 0.92 & 0.03 \\
\hline
\end{tabular}

movement. The percentage of correctly recognized single-DOF and combined-DOF data was analyzed. The thresholds were optimized for each of the methods individually in 1000 steps. For KNFST this resulted in a grid search, since for this method also the kernel width required optimization. It was optimized in steps $\left[2^{-1} \ldots 2^{7}\right]$. For OCSVM, the Gaussian kernel was chosen. The hyperparameters $\nu$ (determining the fraction of data which are allowed to be support vectors) and the bandwidth of the kernel $\sigma$ had to be optimized. Both were varied in grid search in steps of $\left[2^{-10} \ldots 2^{0}\right]$.

\subsubsection{Statistical Analysis}

In order to determine the statistical difference between the investigated methods, a Kruskal-Wallis test was conducted [116]. In case of statistically significant influence of the method, pairwise Wilcoxon rank sum tests with Holm correction [117] were conducted to determine significant differences between the methods. The significance level for all analyses was set to $p<0.05$.

\subsubsection{Results of EDE performance}

The run time of each of the algorithms varied significantly. For estimating all 2160 feature data per run of the single-DOF movements and the 3240 vectors of multi-DOF data, the EDE algorithms required between 0.01s (MD-LDA) and $190 \mathrm{~s}(\mathrm{kNN})$.

The recognition accuracies $P_{\text {single }}\left(P_{\text {comb }}\right)$ were calculated as the conditional probabilities of each feature vector $\boldsymbol{x}$ to be classified as single-DOF (combined-DOF) when it was actually from the subset of single-DOF $\{\mathcal{S}\}$ (combined-DOF $\{\mathcal{C}\}$ ) movements:

$$
\begin{aligned}
P_{\text {single }} & =P(x \mid x \in \mathcal{S}) \\
P_{\text {comb }} & =P(x \mid x \in \mathcal{C})
\end{aligned}
$$

Including all EDE methods, the Kruskal-Wallis analysis revealed significant influence of the method for both $P_{\text {single }}$ and $P_{\text {comb }}, p<10^{-3}$. However, LDA-LL resulted in unacceptable accuracy levels for $P_{\text {comb }}(<30 \%$, see Figure 5.3$)$. Therefore, LDA-LL 


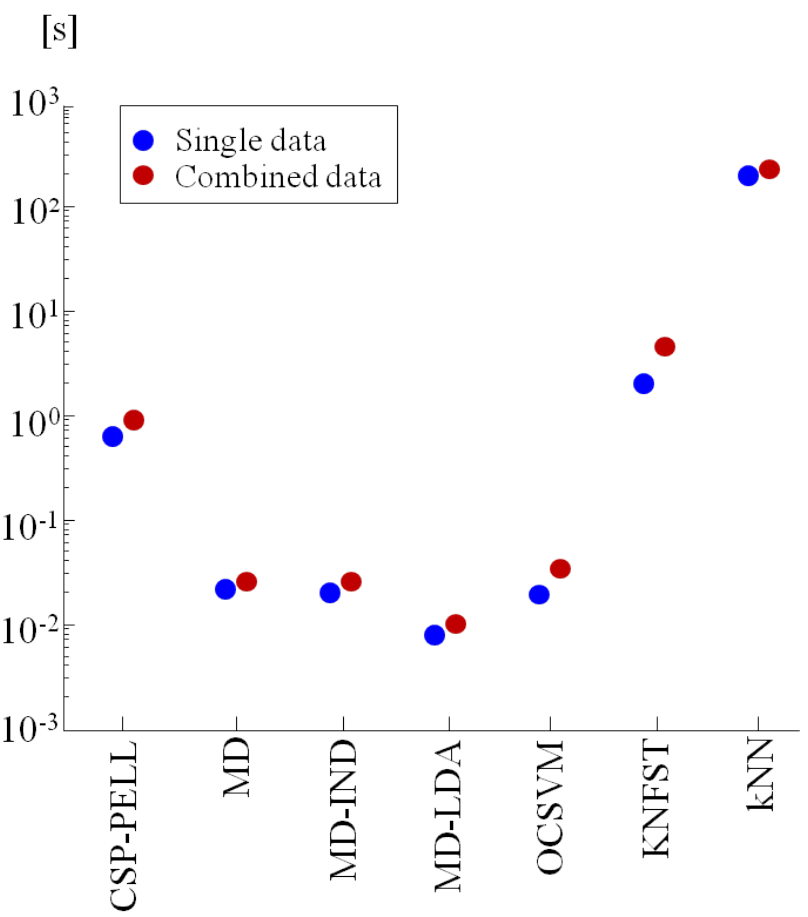

Figure 5.2: Runtimes of EDE methods. Left circles in blue: time in seconds for estimating all 2160 feature vectors of one single-DOF run. Right red circles: the same for 3240 combined data feature vectors. For KNFST only every $4^{\text {th }}$ training vector could be used due to memory constraints.

was excluded from the methods and the analysis was repeated. In this new assessment, $P_{\text {single }}$ was still significantly influenced by the used method $\left(p<10^{-3}\right)$, however the method now had no significant influence anymore on $P_{\text {comb }}(p=0.16)$. In general, the average recognition accuracies were below $95 \%$ for single-DOF data and below $90 \%$ for combined data. This means, $5 \%$ or more of actually single-DOF data were wrongly labeled as combined-DOF data and $10 \%$ or more data were misclassified as single-DOF, when they were actually from the subset of combined movements. Whether this recognition accuracy was sufficient in an online application for smooth control was to be investigated in the online tests (see below). The analysis conducted here allowed for selecting the best suitable EDE candidate for these experiments.

With statistical significance, kNN, MD, MD-IND and MD-LDA outperformed OCSVM and CSP-PELL, but were not significantly different among each other. KNFST was also outperformed, but these differences were not statistically different. The main result obtained in this analysis was hence that the simple to implement and computationally highly efficient methods (based on calculating the Mahalanobis distance) achieved similar better accuracies than the more complex and time intensive methods 
and were thus the methods of choice. The method based on the CSP-PELL would have been of particular interest since it did not require any additional calculations at all (the times shown in Table 5.1 and Figure 5.2 were needed for movements estimation regardless and did not result in any overhead for the estimation of the embedded dimensionality). It was therefore also an interesting candidate for online investigation. However, it achieved significantly worse results compared to the MD based approaches, and therefore had to be discarded. OCSVM achieved relatively poor results and proved to be very sensitive to hyperparameter selection and was therefore not investigated further. KNFST was also not suitable, since it was computationally so expensive (due to the creation of the full kernel matrix) that training data had to be excluded. With this restriction, it did not result in sufficient recognition accuracies. Therefore it had to be excluded from the EDE candidates suitable for online implementation. For its computational complexity, also kNN had to be excluded. Although it yielded good recognition results, it was not superior to the MD based methods.

As a result, the simple MD method was chosen as the most suitable candidate for all further online experiments. The threshold for the method was determined empirically before the start of the experiment, so that both simultaneous and sequential movements could be performed by the subject. After this initial setup, the threshold was not changed anymore.

\subsection{Online control of physical prostheses}

After identifying the optimal EDE method, it was proceeded to integrate the system allowing for simultaneous wrist and sequential hand control in the software framework developed for the study presented in Chapter 4. The main estimation methods, linear regression (LR) and CSP-PE, were already available. The integration of the MD EDE proved to be uncomplicated and its computational efficiency was adequate for real time application. Since the obtained method was a combination of CSP-PE and LR, it will be referred to as CSP-PE+LR from here on.

The online control experiments were largely conducted as those presented in Chapter 4 , with only small alterations. The same prosthetic hand, prosthetic sockets for amputees, training and data recording paradigm, signal processing, evaluation schemes and study design, including real time control of a physical prosthesis in applied tasks, 
Single Accuracy

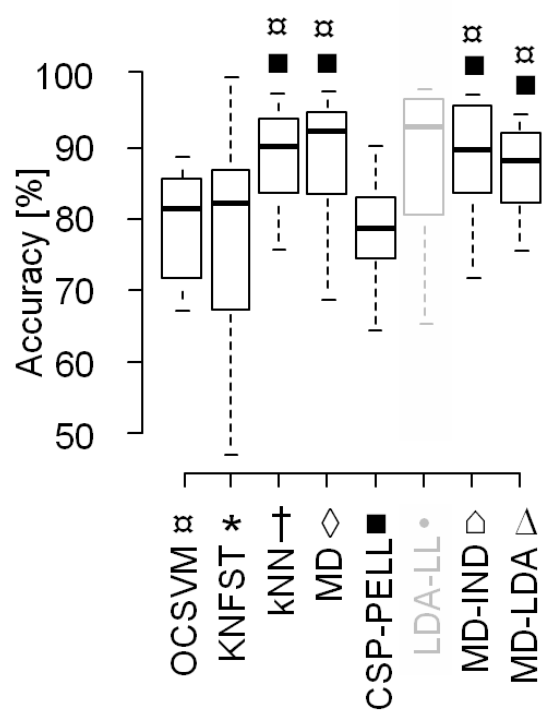

Combined Accuracy

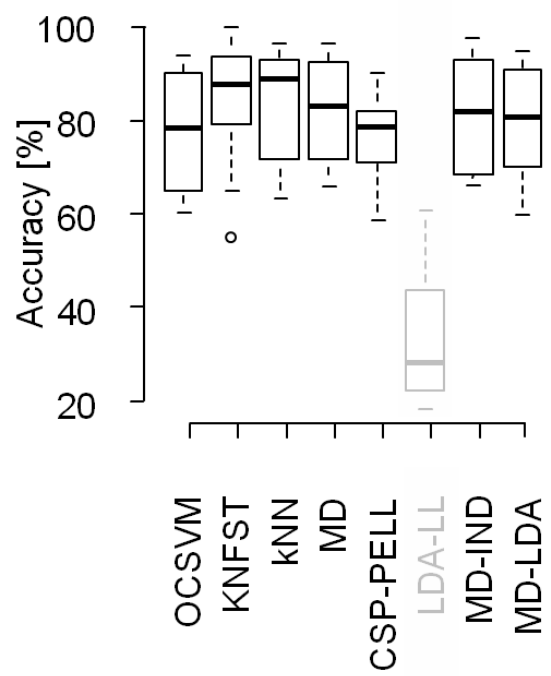

Figure 5.3: Accuracies of EDE methods. Left: single-DOF accuracy - the percentage of single-DOF movements recognized as such. Right: combined-DOF accuracy: Percentage of combined-DOF data recognized as such. It is important to consider both accuracies at the same time. Due to its bad performance in the combined accuracy, LDA-LL had to be excluded. Significant differences between methods are marked with their respective symbols.

were used. For the experiments, the two amputee subjects Amp1 and Amp2 of the study in Chapter 4, which were capable of controlling all 8 prosthetic movements, were recruited to participate in this study, since the other two subjects were not capable of performing WF and WE independently from all other movements required. In addition to the amputee subjects, 5 of the healthy subjects which had already completed the study described in Chapter 4 were included. Amputee subjects were asked to complete the Southampton hand assessment procedure (SHAP) test [118], a comprehensive test particularly developed for the assessment of upper limb prosthesis functionality in amputees. It is a test investigating prosthetic function in situations close to those of activities of daily living (ADL) and was thus particularly suited for the evaluation of the natural, dexterous prosthesis control facilitated by the simultaneous, proportional control used in this study. The test procedures included pouring a cup of water, cutting a piece of plastic modeling mass with a knife, opening buttons on clothes, picking up coins from a table top, etc. For all tests and the exact test procedure, the interested reader is referred to [118]. The SHAP test was performed by trained clinicians of the AKH Vienna General Hospital.

With the able-bodied subjects, it was preferred to repeat the box and blocks test, 
clothes pin test and block turn test, since ADL testing was considered not as relevant as with amputees and direct comparison of the applied method to the purely sequential control of Chapter 4 was favored. The tests with able-bodied subjects were repeated 6 months after the initial tests. In the meantime, these subjects had not participated in any machine learning based prosthetic control tasks. In this setting of tests with able-bodied and amputee subjects, a comprehensive evaluation of the investigated method was facilitated.

\subsection{Results}

All able-bodied and amputee subjects were able to complete the respective tests conducted with each subject group. In the following, the detailed results for each subject group are presented.

\subsubsection{Amputee SHAP results}

The SHAP results of amputee Amp1 are detailed in Table 5.2. The overall score of function was 58 for this subject. During the entire SHAP test, in Amp1, 20.1\% of all rotation movements were also combined with flexion/extension of the wrist, whereas $24.5 \%$ of all wrist flexion/extensions were combined with a rotation activation. Exemplary activations for Amp1 are shown in Figure 5.4.

For Amp2, 38.2\% of all rotations were combined with wrist flexion/extension, and flexion/extension was combined with rotation $27.1 \%$ of all times, hence this subject used simultaneous activations more frequently than in the first subject. This was likely the case since, as described in Chapter 4, this subject was also capable of performing combined movements (although different ones than used in this study) with his everyday prosthesis. Amp2 also achieved better results than Amp1 in the SHAP test. The overall score of Amp2 was 71. The detailed results are shown in Table 5.3.

Unfortunately, the exact way the global index of function score is calculated for the SHAP test is not revealed to the user. The measured times per task have to be inserted in a web-based form, and the index of function is computed automatically. The test is standardized so that 100 points represent results equal to normally limbed subjects. In order to gain more insight on how the subjects performed in direct comparison to each other, their time results for each subtest of the SHAP were plotted against 
Table 5.2: SHAP test results for amputee subject Amp1

\begin{tabular}{|c|c|c|c|}
\hline \multicolumn{4}{|c|}{ Abstract Objects } \\
\hline Light Sphere: & $2.66 \mathrm{~s}$ & Heavy Sphere: & $4.78 \mathrm{~s}$ \\
\hline Light Tripod: & $3.56 \mathrm{~s}$ & Heavy Tripod: & $3.53 \mathrm{~s}$ \\
\hline Light Power: & $3.25 \mathrm{~s}$ & Heavy Power: & $3.22 \mathrm{~s}$ \\
\hline Light Lateral: & $2.81 \mathrm{~s}$ & Heavy Lateral: & $5.31 \mathrm{~s}$ \\
\hline Light Tip: & $2.88 \mathrm{~s}$ & Heavy Tip: & $4.47 \mathrm{~s}$ \\
\hline Light Extension: & $3.88 \mathrm{~s}$ & Heavy Extension: & $4.88 \mathrm{~s}$ \\
\hline \multicolumn{4}{|c|}{ Activities of Daily Living } \\
\hline Pick Up Coins: & $22.25 \mathrm{~s}$ & Lifting a Heavy Object: & $10.37 \mathrm{~s}$ \\
\hline Button Board: & $35.20 \mathrm{~s}$ & Lifting a Light Object: & $4.15 \mathrm{~s}$ \\
\hline Simulated Food Cutting: & $22.47 \mathrm{~s}$ & Lifting a Tray: & $7.25 \mathrm{~s}$ \\
\hline Page Turning: & $11.97 \mathrm{~s}$ & Rotate Key: & $4.25 \mathrm{~s}$ \\
\hline Jar Lid: & $3.93 \mathrm{~s}$ & Open/Close Zip: & $10.59 \mathrm{~s}$ \\
\hline Glass Jug Pouring: & $12.37 \mathrm{~s}$ & Rotate A Screw: & $25.31 \mathrm{~s}$ \\
\hline Carton Pouring: & $11.35 \mathrm{~s}$ & Door Handle: & $3.53 \mathrm{~s}$ \\
\hline \multicolumn{4}{|c|}{ SHAP Scores } \\
\hline \multicolumn{4}{|l|}{ Functionality Profile } \\
\hline Spherical: & 85.00 & Tripod: & 40.00 \\
\hline Power: & 45.00 & Lateral: & 74.00 \\
\hline Tip: & 56.00 & Extension: & 51.00 \\
\hline \multicolumn{4}{|c|}{ Index of Function Score } \\
\hline Index of Function: & 58.00 & & \\
\hline
\end{tabular}




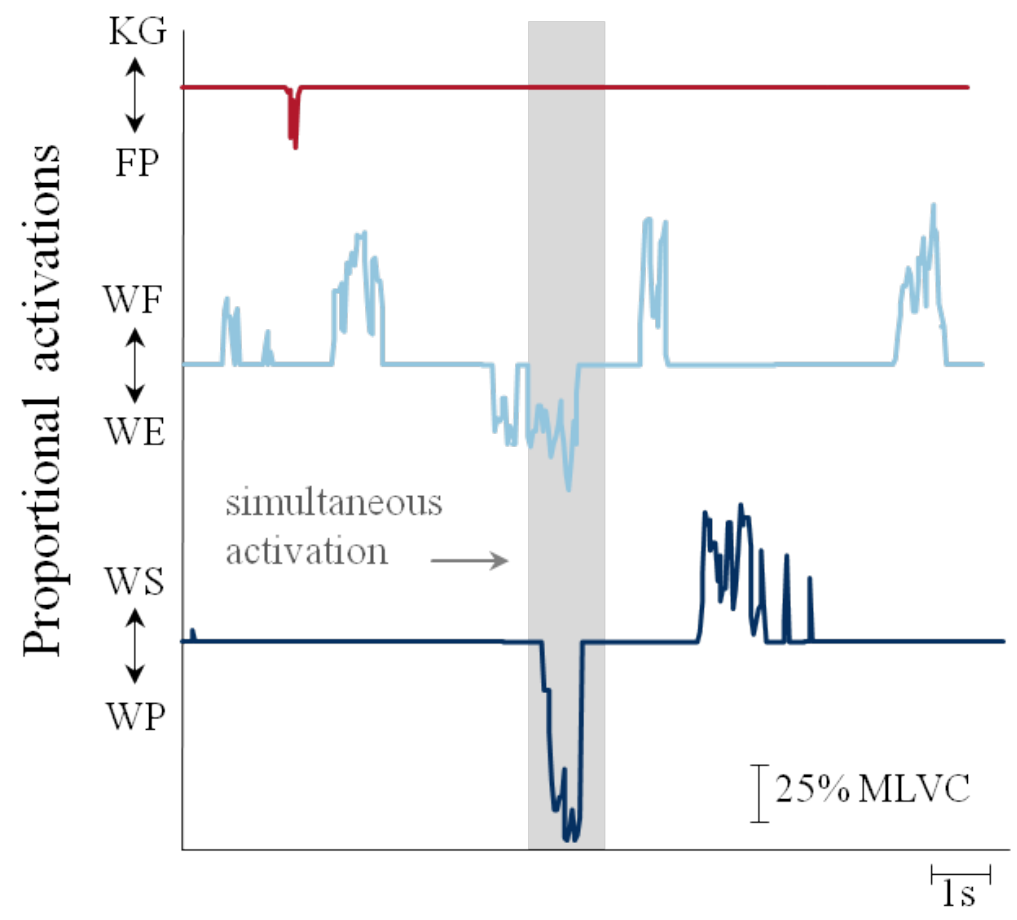

Figure 5.4: Exemplary activations of the first amputee participating in the SHAP test. It is shown that the subject chose to activate most of the movements sequentially, but also simultaneous movements were possible and used by the subject (highlighted in gray).

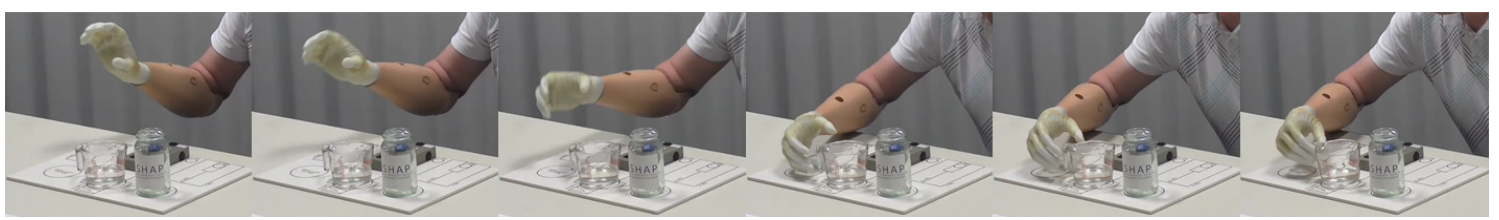

Figure 5.5: Exemplary sequence of Amp2 performing simultaneous wrist flexion and supination followed by a pinch grip to grasp the mug of the SHAP test. 
Table 5.3: SHAP test results for amputee subject Amp2

\begin{tabular}{|c|c|c|c|}
\hline \multicolumn{4}{|c|}{ Abstract Objects } \\
\hline Light Sphere: & $3,60 \mathrm{~s}$ & Heavy Sphere: & $4.03 \mathrm{~s}$ \\
\hline Light Tripod: & $3,94 \mathrm{~s}$ & Heavy Tripod: & $3.98 \mathrm{~s}$ \\
\hline Light Power: & $3,22 \mathrm{~s}$ & Heavy Power: & $3.50 \mathrm{~s}$ \\
\hline Light Lateral: & $4.66 \mathrm{~s}$ & Heavy Lateral: & $5.20 \mathrm{~s}$ \\
\hline Light Tip: & $3.75 \mathrm{~s}$ & Heavy Tip: & $4.59 \mathrm{~s}$ \\
\hline Light Extension: & $3.08 \mathrm{~s}$ & Heavy Extension: & $4.22 \mathrm{~s}$ \\
\hline \multicolumn{4}{|c|}{ Activities of Daily Living } \\
\hline Pick Up Coins: & $26.82 \mathrm{~s}$ & Lifting a Heavy Object: & $4.53 \mathrm{~s}$ \\
\hline Button Board: & $19.80 \mathrm{~s}$ & Lifting a Light Object: & $2.80 \mathrm{~s}$ \\
\hline Simulated Food Cutting: & $30.10 \mathrm{~s}$ & Lifting a Tray: & $2.55 \mathrm{~s}$ \\
\hline Page Turning: & $6.96 \mathrm{~s}$ & Rotate Key: & $4.97 \mathrm{~s}$ \\
\hline Jar Lid: & $4.40 \mathrm{~s}$ & Open/Close Zip: & $6.47 \mathrm{~s}$ \\
\hline Glass Jug Pouring: & $13.12 \mathrm{~s}$ & Rotate A Screw: & $11.62 \mathrm{~s}$ \\
\hline Carton Pouring: & $17.97 \mathrm{~s}$ & Door Handle: & $2.59 \mathrm{~s}$ \\
\hline \multicolumn{4}{|c|}{ SHAP Scores } \\
\hline \multicolumn{4}{|l|}{ Functionality Profile } \\
\hline Spherical: & 78.00 & Tripod: & 38.00 \\
\hline Power: & 54.00 & Lateral: & 76.00 \\
\hline Tip: & 54,00 & Extension: & 74,00 \\
\hline \multicolumn{4}{|c|}{ Index of Function Score } \\
\hline Index of Function: & 71.00 & & \\
\hline
\end{tabular}




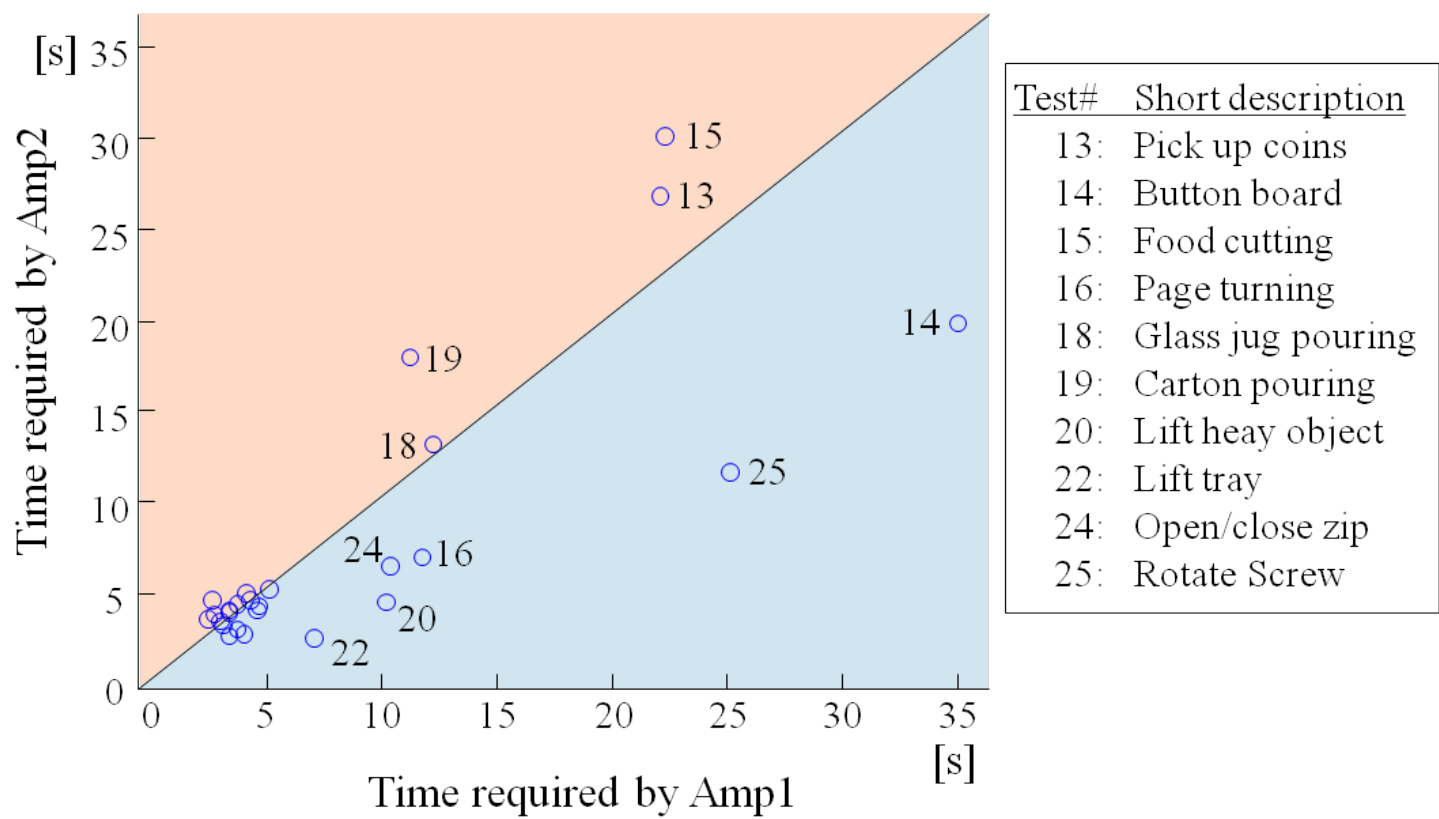

Figure 5.6: Direct comparison for each SHAP test result between the two amputees participating in this study. The upper, red half of the plot represents tests were Amp1 performed faster than Amp2, while in the lower, blue half Amp2 was faster than Amp1. For tests with substantial differences between the subjects, the SHAP test number is added next to the data point.

each other in a scatter plot, see Figure 5.6. In this plot it is shown, that simple pick-and-place tasks were scattered around $4 \mathrm{~s}$ and were equally distributed among the two subjects. The more complex tasks are numbered for identification. Generally, Amp2 performed better than Amp1 in 6 out of these 10 difficult tasks, with two of these differences being remarkably different: $11.62 \mathrm{~s}$ vs. $25.31 \mathrm{~s}$ for rotating the screw and $19.80 \mathrm{~s}$ vs. $35.20 \mathrm{~s}$ for the button board. In summary, the differences visualized in Figure 5.6 likely explain the different outcome scores of 58 for Amp1 and 71 for Amp2, although with only two exceptions these differences did not appear particularly prominent.

\subsubsection{Able-bodied results}

Since in this test only one control method was tested, subjects did not report muscle fatigue and all were able to complete the tests. A t-test was conducted to determine statistically significant differences between CSP-PE and CSP-PE+LR. In the box and blocks test, the subjects needed $3.11 \pm 0.62 \mathrm{~s}$ on average to transfer each block with CSP-PE+LR. Compared to the $3.25 \pm 0.62 \mathrm{~s}$ required with CSP-PE, this difference was not statistically significant $(p=0.52)$. In the clothes spin test, subjects improved 
their completion times from an average of $23.4 \pm 4.66 \mathrm{~s}$ with CSP-PE to $16.4 \pm 3.35 \mathrm{~s}$ with CSP-PE $+\mathrm{LR}$, which was also statistically significant $\left(p<10^{-3}\right)$. The same trend was observed in the block turn test, where subjects improved from $23.2 \pm 4.94 \mathrm{~s}$ to $16.0 \pm 3.59 \mathrm{~s}$ with the CSP-PE + LR method, which was again statistically significant $\left(p<10^{-3}\right)$. The number of drops of the block slightly increased with CSP-PE+LR compared to CSP-PE, from a total of 1 to 3 drops in all 15 experiments ( 5 subjects, 3 test repetitions per subject), which can still be considered as a good performance and was not statistically different between methods.

During all tests, able-bodied subjects combined $14.5 \%$ of all rotation movements with wrist flexion/extension, and $26.7 \%$ of all flexion/extensions were combined with rotations.

\subsection{Study discussion and conclusion}

In this study a novel concept for combining estimators has been presented and evaluated. In the analysis of simultaneous and sequential regressors of previous studies, complimentary benefits and compromises of these two estimator types were identified. Therefore it was hypothesized that by combining these methods, each specialist could potentially alleviate the shortcomings of the respective other method, resulting in an overall improved control system, exhibiting favorable behavior compared to each of the methods used alone.

The system investigated in this study facilitated the control over 8 prosthetic functions, 4 of which could also be used in a simultaneous, proportional manner. Here, wrist rotation and wrist flexion/extension where simultaneously controllable. However, it is noteworthy that the same methodology is also applicable to the control of less functions, for example for users who do not have the possibility of using an actuated wrist flexion/extension unit (these are not commercially available on the market yet). In this case, for example hand open/close and rotation could be combined in the same manner, which could readily be useful for commercial prostheses. However, this has not been investigated in this study.

The most crucial step of this system, as highlighted in Figure 5.1, was the estimator, which in the first step determined whether a movement sEMG feature vector should be forwarded to the specialist for sequential or simultaneous estimation. To accomplish this task, eight suitable candidates for this purpose were identified. In the direct 


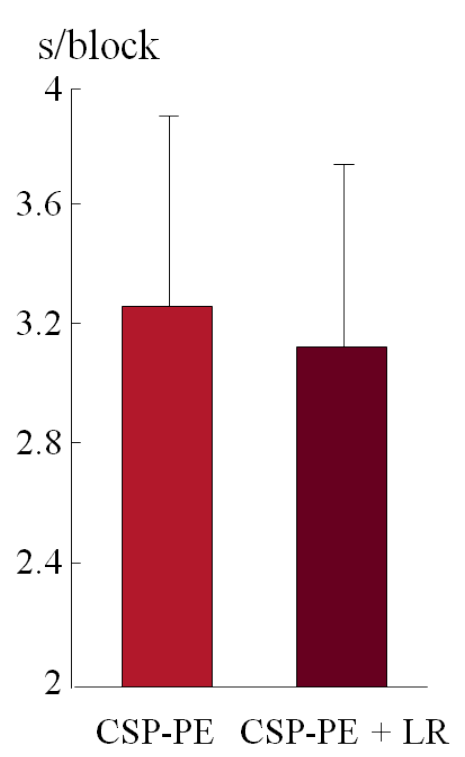

(a) Box and blocks

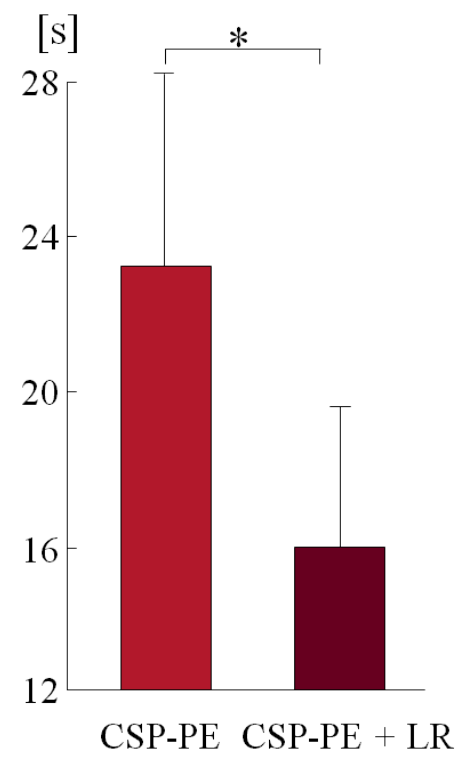

(c) Block turn - Time

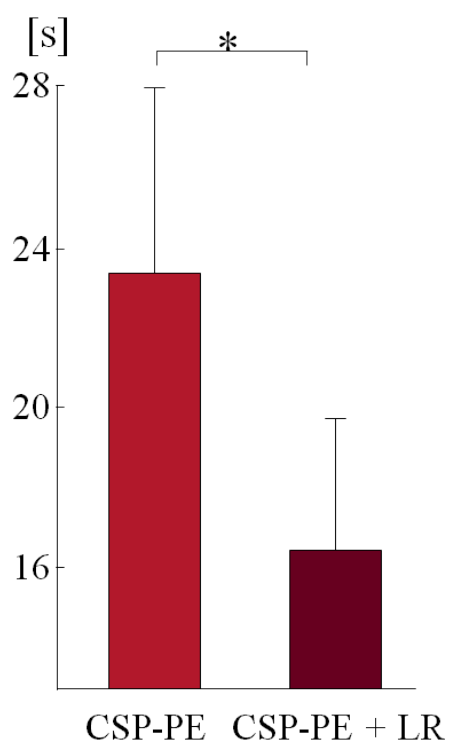

(b) Clothes pin

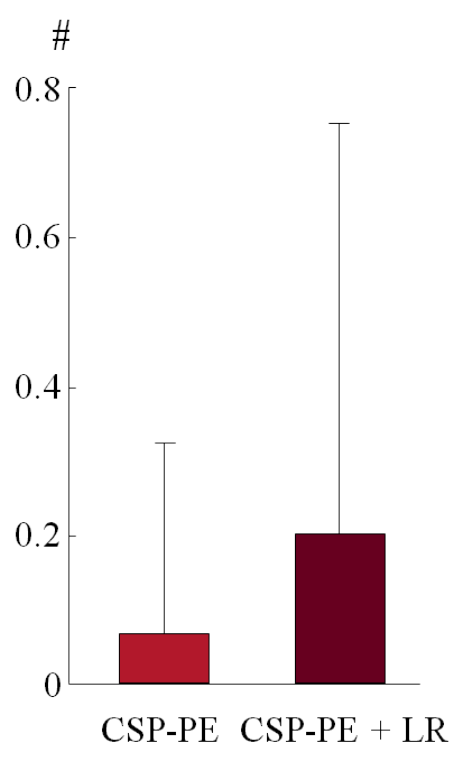

(d) Block turn - Drops

Figure 5.7: Results of CSP-PE+LR compared to CSP-PE. In the simple box and blocks test, the advanced control mechanism with simultaneous wrist movements did not result in a significantly different performance compared to the sequential CSP-PE, since only hand open and close were required. However, in the two more difficult tasks, which required activation over several DOF, the simultaneous wrist and sequential hand activation control method resulted in a significant performance increase. ${ }^{*}$ denotes significant difference $(p<0.05)$. 
comparison of these methods, one of the simplest methods based on the thresholded Mahalanobis distance to the training data proved to be also one of the most accurate and was thus chosen for the online experiments. During the online control of the physical prosthesis, it was found that empiric determination of the appropriate threshold was straightforward, however the optimal values varied considerably between subjects (from 18 to 40 a.u.). In future developments, this threshold should be computed for each subject individually and automatically, although this will be a challenging task, since no data of simultaneous movements are available for optimization.

The results of the amputee subjects achieved in the SHAP test were encouraging, however especially the result of Amp1 (58 points) was inferior to the expected outcome $[119,120]$. A closer analysis and comparison revealed that two of the 26 subtests (button board and rotate screw) were substantially different between the two subjects, potentially explaining the relatively big difference in the overall outcome score.

It was further found that subjects used the simultaneous wrist movements to different extents, with Amp1 almost twice as much as Amp2. This is likely explained by the fact that Amp2 is also capable of using simultaneous movements with his personal prosthesis (although different ones as used in this study), whereas Amp1 had not used simultaneous movements before. It can therefore be speculated that user training and usage habits play an important role in the amount of exploiting the capabilities of simultaneous wrist movements. This interesting observation should be investigated more closely in a dedicated study, preferably in a longitudinal scope to allow better subject familiarization with the control.

In able-bodied subjects, substantial improvements with CSP-PE+LR over purely sequential CSP-PE alone were obtained. This could partially be explained by the usage of simultaneous movements in about $20 \%$ of all wrist movements. Additionally to the time gained by simultaneous movements, the CSP-PE+LR based system was less affected by "unclean" execution of wrist movements. For example, if the subject wanted to move the prosthetic hand in full supination and wrist extension position, with CSP-PE first activating e.g. supination was required. Then the subject had to rotate his arm from which the EMG signals were collected, back to the neutral position (in which the training data were recorded) and perform the wrist extension. In case the return to the neutral position was omitted between the two movements, CSP-PE would not have recognized the movement and not issued a control command to the prosthesis. However, in CSP-PE+LR, this would still result in a good 
estimation by LR, allowing the prosthesis to reach the desired orientation (without necessarily causing a simultaneous movement, when the prosthesis was already in full supination prior to the subject starting the wrist extension). This behavior was observed multiple times by the experimenter in all subjects. Furthermore, able-bodied subjects reported difficulties in determining the correct combination of wrist movements for aligning the prosthesis in the desired orientation. These problems were not reported by the amputee subjects, who had less difficulties to incorporate the current prosthesis position into their body image than the able-bodied subjects and thus to perform the correct combined movements. This may also partially be attributed to the fact that able-bodied subjects had to use the sEMG of their right forearms to control the prosthesis on their left hand, which was (as described in Chapter 4) imposed by the mechanical constraints of the used equipment. While this drawback proved unproblematic for sequential control in the study presented in Chapter 4, in the present study using combined movements this setting might have had bigger influence. In future studies with able-bodied subjects using combined wrist motions, this constrained should be eliminated by suitable hardware. However, despite these difficulties, able-bodied subjects achieved very good results in these experiments with CSP-PE+LR. It outperformed the purely sequential CSP-PE as used in Chapter 4, which proved to be the best method in that study. The experiments of this study were conducted 6 months after those of the study of Chapter 4 . In the meantime, none of the subjects had acquired further experiences with the machine learning based realtime control of prosthesis, making the improved results unlikely to be due to carry over effects [121]. The results are therefore encouraging and further investigations considering the improvements mentioned above are warranted.

\section{Conclusion}

In this study, the combination of simultaneous and sequential proportional estimators was developed and tested in real-time control experiments of a physical prosthesis in applied tests with both able-bodied and amputee subjects. For amputees the same custom sockets for attaching the prosthesis as described in the study of Chapter 4 were used, maximizing the clinical relevance of the test protocol and the achieved results. The simultaneous control of the wrist proved to be advantageous over pure sequential control, however especially for able-bodied subjects, the contra-lateral control strategy was identified as an important issue to be resolved in future studies for improved 
intuitiveness of the simultaneous control in this subject group. Also, in future studies directly comparable test results (e.g. SHAP test) of pure sequential control and mixed simultaneous and sequential control should be obtained to quantify the benefit of simultaneous wrist motions over sequential control more extensively. 


\section{$6 \quad$ Thesis discussion and conclusion}

In the beginning of thesis, the general goals of machine learning based myoelectric control of multifunctional prostheses of the upper extremity and the related challenges were discussed. A substantial lack of robustness of the previously proposed methods was identified. Therefore, in the further progress of this thesis, a series of four studies was dedicated to investigate these matters profoundly and propose several solutions. The particular focus was put on the robustness of these systems, advancing the state of the art in prosthesis control.

In the first study, a previously insufficiently described influence of non-stationarity on the sEMG signals was identified and an attempt to provide deeper insights was made: the influence of time. To this end, in total 11 subjects divided in able-bodied and amputee groups, were recruited to participate in this study over the course of 5 successive days. A well accepted pattern recognition method was investigated regarding its performance of discriminating 8 wrist and hand movements over the course of this time. The analyses were carried out in an offline manner for exhaustive tests. It was revealed that the more days lay between the recording of the training and the testing data of the classifier, the more mis-classifications occurred. This is relevant knowledge for the clinical application of machine learning based methods for prosthesis control. Similar work had only been conducted before by [80], but this work was limited to one healthy subject. In [122] a study was conducted across 4 days, but again limited to able-bodied subjects and no inter-day testing was performed. Therefore, in this work important new information was gained. The study was however limited to offline analyses. In future studies, it should be investigated if the effects observed here translate to online control. Perhaps, the user could compensate 
for some mis-classifications by slightly changing his movement strategies. However, the results of this work point at the probable necessity of such user (or algorithm) adaptations, which is a valuable information.

In the third chapter, a novel methodology for alleviating the observed results described in Chapter 2 was explored. A self-correction mechanism was designed, described and analyzed. The proposed system effectively facilitated the autonomous detection of mis-classifications and allowed for their correction. With this method, a significantly increased performance was obtained when compared to the base line of no correction or when three previously proposed correction methods were applied. As the main result of this study, a solution for increasing the robustness of sEMG signal classification was proposed. A further result of interest was that the suppression of wrong movements (i.e. predicting the rest class in case of uncertain estimations) could be beneficial for removing false prosthetic motions. However, due to the offline nature of this study, this had to be confirmed in the next study. Similar findings were reported by Scheme et al. [52], but due to its limitation to an abstract cursor control task, the transferability of the results to the control of a physical prostheses remained unknown. This issue was also addressed in the next study.

In order to substantially extend the findings described in Chapter 3, a third study was designed to investigate the beneficial behavior of suppressing wrong movements in an online control study using physical prostheses, described in Chapter 4. For this purpose, four amputees were fitted with experimental prosthetic sockets, suitable for machine learning based prosthesis control. Additionally, 10 able-bodied subjects were fitted with an experimental attachment to use a prosthesis with a sound limb. With this realistic setup of controlling physical prostheses in real time, some clinically relevant tests were conducted. In order to transfer the previously gained knowledge on wrong movement suppression to regression based algorithms, a novel proportional estimator was developed, which possessed this capability. This new method was compared to two other, state-of-the-art control schemes without such corrections. It was shown that the novel estimator outperformed both other methods in both subject groups. Due to the clinically highly relevant test scenarios of real prosthetic control in applied online object manipulation tasks, these results were encouraging and will potentially have great influence on the future development of upper limb pros- 
thesis. To the knowledge of the author, this was the first time that such extensive real-time control investigations with physical prostheses and custom manufactured sockets for each participant have been conducted. One drawback of the novel method was however that it did not allow simultaneous control of several DOF. This issue was addressed in a new, dedicated study.

In Chapter 5, the system conceived, described and tested in Chapter 4 was extended to permit simultaneous proportional control of two of the involved DOF. Similar and extended tests compared to those shown in Chapter 4 were performed, however now exploiting simultaneous wrist control, augmented by sequential hand activations. In the online control of the prosthesis, this enhancement of dexterity proved to be beneficial, especially for experienced subjects. Unfortunately, none of the subjects had the possibility to use the system for a longer period of time for getting better acquainted with the control. This will be the focus of future studies, in order to investigate the factor of user learning. This system is the final product of this thesis. It incorporates the important knowledge gained in all the investigations described before in this thesis. It exhibits many desirable properties such as robustness, control over many DOF, simultaneous and proportional activations for natural and fluent control, improved fine control and gross positioning of the end effector and computational efficiency, making it real-time capable. The system was tested extensively with able-bodied and amputee users. The subjective feedback from the participants was very positive, underlining the quantitative improvements as measured by the applied tests. For commercial exploitation of the described system, several steps still need to be taken, such as reducing hardware costs of multi-electrode sockets, clinicians and physiotherapist education and advanced user training guide lines including optimized feedback for effective training. Further, take-home test prostheses for selected users should be issued for gathering more evidence of the usefulness of the proposed method for and by end users.

All chapters and studies presented within this thesis followed the goal of increasing the robustness and clinical viability of myoelectric controlled multifunctional prostheses. The scopes of the studies were successively extended from classification to regression and from offline to online control of physical prosthesis in close to real life prosthetic usage scenarios. In conclusion, the obtained results will potentially provide 
guidelines, which are important for the successful commercialization of dexterous, intuitively controlled multifunctional prostheses, in order to reach the best achievable results for prosthetic users. 


\section{Bibliography}

[1] K. Ziegler-Graham, E. J. MacKenzie, P. L. Ephraim, T. G. Travison, and R. Brookmeyer, "Estimating the prevalence of limb loss in the United States: 2005 to 2050." Archives of Physical Medicine and Rehabilitation, vol. 89, no. 3, pp. 422-9, Mar. 2008.

[2] R. Braddom, Physical Medicine and Rehabilitation, 4th ed. Elsevier, 2011.

[3] T. R. Dillingham, L. E. Pezzin, and E. J. MacKenzie, "Incidence, acute care length of stay, and discharge to rehabilitation of traumatic amputee patients: an epidemiologic study." Archives of Physical Medicine and Rehabilitation, vol. 79, no. 3, pp. 279-87, Mar. 1998.

[4] C. A. Krueger, J. C. Wenke, and J. R. Ficke, "Ten years at war: comprehensive analysis of amputation trends." The Journal of Trauma and Acute Care Surgery, vol. 73, no. 6 Suppl 5, pp. S438-44, Dec. 2012.

[5] M. Lanzetta, J.-M. Dubernard, and P. Petruzzo, Eds., Hand Transplantation, xxi ed. Springer, 2007.

[6] C. W. Martin and D. Edeer, "Upper Limb Prostheses A Review of the Literature With a Focus on Myoelectric Hands," WorkSafeBC Evidence Based Practice Group, no. February, 2011.

[7] R. Merletti and P. A. Parker, Eds., Electromyography: Physiology, Engineering and Noninvasive Applications. Piscataway, NJ: John Wiley \& Sons, Inc., 2004.

[8] M. Ortiz-Catalan, R. Brånemark, B. Håkansson, and J. Delbeke, "On the viability of implantable electrodes for the natural control of artificial limbs: review and discussion." Biomedical Engineering Online, vol. 11, p. 33, Jan. 2012. 
[9] L. J. Hargrove, K. Englehart, and B. Hudgins, "A comparison of surface and intramuscular myoelectric signal classification." IEEE Transactions on Biomedical Engineering, vol. 54, no. 5, pp. 847-53, May 2007.

[10] E. N. Kamavuako, J. C. Rosenvang, R. Horup, W. Jensen, D. Farina, and K. B. Englehart, "Surface Versus Untargeted Intramuscular EMG Based Classification of Simultaneous and Dynamically Changing Movements." IEEE Transactions on Neural Systems and Rehabilitation Engineering, no. c, pp. 1-8, Mar. 2013.

[11] T. R. Farrell and R. F. F. Weir, "A comparison of the effects of electrode implantation and targeting on pattern classification accuracy for prosthesis control." IEEE Transactions on Biomedical Engineering, vol. 55, no. 9, pp. 2198-211, Sep. 2008.

[12] B. Cronholm, "Phantom limbs in amputees; a study of changes in the integration of centripetal impulses with special reference to referred sensations." Acta Psychiatrica et Neurologica Scandinavica. Supplementum, vol. 72, pp. 1-310, Jan. 1951.

[13] A. Gharabaghi, G. Naros, A. Walter, A. Roth, M. Bogdan, W. Rosenstiel, C. Mehring, and N. Birbaumer, "Epidural electrocorticography of phantom hand movement following long-term upper-limb amputation." Frontiers in human neuroscience, vol. 8, no. May, p. 285, Jan. 2014.

[14] E. Biddiss and T. Chau, "Upper-limb prosthetics: critical factors in device abandonment." American journal of physical medicine $\&$ rehabilitation / Association of Academic Physiatrists, vol. 86, no. 12, pp. 977-87, Dec. 2007.

[15] L. Philipson, D. Childress, and J. Strysk, "Digital approaches to myoelectric state control of prostheses," Bulletin of Prosthetics Research, vol. 18, no. 2, pp. 3-11, 1981.

[16] D. Dorcas and R. N. Scott, "A three state myoelectric control," Medical \&6 Biological Engineering \& Computing, vol. 4, pp. 367-370, 1966.

[17] "Otto Bock Upper Limb Prostheses Catalogue." [Online]. Available: Http: //www.ottobock.com/cps/rde/xbcr/ob_com_en/646K6-GB-03-1205w.pdf 
[18] R. Reiter, "Eine neue Elektrokunsthand," Grenzegbiete der Medizin, vol. 4, no. 133, 1948.

[19] D. S. Childress, "Historical Aspects of Powered Limb Prostheses," Clinical Prosthetics and Orthotics, vol. 9, no. 1, pp. 2-13, 1985.

[20] C. K. Battye, A. Nightingale, and J. Whillis, "The Use of Myo-Electric Currents in the Operation of Prostheses," Journal of Bone and Joint Surgery, vol. 37B, no. 3, pp. 506-510, 1955.

[21] A. Kobrinskii, S. Bolkhoivin, L. Voskoboinikova, D. Joffe, E. Polyan, Y. L. Slavictskü, A. Y. Sysin, and S. Yakobsen, Ya, "Problems of Bioelectric Control," in International Federation on Automatic Control Conference, Moscow, 1960, pp. 1119-22.

[22] J. T. Belter, J. L. Segil, A. M. Dollar, and R. F. Weir, "Mechanical design and performance specifications of anthropomorphic prosthetic hands: a review." Journal of Rehabilitation Research and Development, vol. 50, no. 5, pp. 599618, Aug. 2013.

[23] F. R. Finley and R. W. Wirta, "Myocoder-computer study of electromyographic patterns." Archives of Physical Medicine and Rehabilitation, vol. 48, no. 1, pp. 20-4, Jan. 1967.

[24] D. Graupe and W. Cline, "Functional Separation of EMG Signals via ARMA Identification Methods for Prosthesis Control Purposes," IEEE Transactions on Systems, Man, and Cybernetics, vol. SMC-5, no. 2, pp. 252-259, 1975.

[25] P. Herberts, A. C., and K. Caine, "Clinical Application Study of Multifunctional Prosthetic Hands," The Journal of Bone and Joint Surgery. British volume, vol. 60-B, no. 4, pp. 552-560, 1978.

[26] B. Hudgins, P. Parker, and R. N. Scott, "A new strategy for multifunction myoelectric control." IEEE Transactions on Biomedical Engineering, vol. 40, no. 1, pp. 82-94, Jan. 1993.

[27] K. Englehart and B. Hudgins, "A robust, real-time control scheme for multifunction myoelectric control." IEEE Transactions on Biomedical Engineering, vol. 50, no. 7, pp. 848-54, Jul. 2003. 
[28] B. Peerdeman, D. Boere, H. Witteveen, R. Huis in 'tVeld, H. Hermens, S. Stramigioli, H. Rietman, P. Veltink, and S. Misra, "Myoelectric forearm prostheses: State of the art from a user-centered perspective," The Journal of Rehabilitation Research and Development, vol. 48, no. 6, p. 719, 2011.

[29] M. Zardoshti-Kermani, B. Wheeler, K. Badie, and R. Hashemi, "EMG feature evaluation for movement control of upper extremity prostheses," IEEE Transactions on Rehabilitation Engineering, vol. 3, no. 4, pp. 324-333, 1995.

[30] M. Zecca, S. Micera, M. C. Carrozza, and P. Dario, "Control of multifunctional prosthetic hands by processing the electromyographic signal." Critical Reviews in Biomedical Engineering, vol. 30, no. 4-6, pp. 459-85, Jan. 2002.

[31] M.-F. Lucas, A. Gaufriau, S. Pascual, C. Doncarli, and D. Farina, "Multichannel surface EMG classification using support vector machines and signalbased wavelet optimization," Biomedical Signal Processing and Control, vol. 3, no. 2, pp. 169-174, Apr. 2008.

[32] A. Maitrot, M. F. Lucas, C. Doncarli, and D. Farina, "Signal-dependent wavelets for electromyogram classification." Medical $\& 3$ Biological Engineering E Computing, vol. 43, no. 4, pp. 487-92, Jul. 2005.

[33] R. Boostani and M. H. Moradi, "Evaluation of the forearm EMG signal features for the control of a prosthetic hand." Physiological Measurement, vol. 24, no. 2, pp. 309-19, May 2003.

[34] M. Oskoei and H. Hu, "Support Vector Machine-Based Classication Scheme for Myoelectric Control Applied to Upper Limb," IEEE Transactions on Biomedical Engineering, vol. 55, no. 8, pp. 1956-1965, 2008.

[35] M. Khezri and M. Jahed, "Real-time intelligent pattern recognition algorithm for surface EMG signals." Biomedical Engineering Online, vol. 6, no. 45, pp. 1-12, Jan. 2007.

[36] K. Englehart, B. Hudgins, P. a. Parker, and M. Stevenson, "Classification of the myoelectric signal using time-frequency based representations." Medical Engineering $\& 3$ Physics, vol. 21, pp. 431-8, 1999. 
[37] G. R. Naik, D. K. Kumar, and S. Arjunan, "Use of sEMG in identification of low level muscle activities: features based on ICA and fractal dimension." Conference Proceedings of the Annual International Conference of the IEEE Engineering in Medicine and Biology Society., vol. 2009, pp. 364-7, Jan. 2009.

[38] S. Theodoridis and K. Koutroumbas, Pattern Recognition, 4th ed. Academic Press, Sep. 2008.

[39] K. Fukunaga, Introduction to Statistical Pattern Recognition, 2nd ed. San Diego, CA, USA: Academic Press, 1990.

[40] L. Hargrove, K. Englehart, and B. Hudgins, "A training strategy to reduce classification degradation due to electrode displacements in pattern recognition based myoelectric control," Biomedical Signal Processing and Control, vol. 3, no. 2, pp. 175-180, Apr. 2008.

[41] L. J. Hargrove, E. J. Scheme, K. B. Englehart, and B. S. Hudgins, "Multiple binary classifications via linear discriminant analysis for improved controllability of a powered prosthesis." IEEE Transactions on Neural Systems and Rehabilitation Engineering, vol. 18, no. 1, pp. 49-57, Feb. 2010.

[42] N. Jiang, S. Muceli, B. Graimann, and D. Farina, "Effect of arm position on the prediction of kinematics from EMG in amputees." Medical $\&$ Biological Engineering $\&$ Computing, vol. 51, no. 1-2, pp. 143-51, Oct. 2013.

[43] J. L. G. Nielsen, S. Holmgaard, N. Jiang, K. B. Englehart, D. Farina, and P. a. Parker, "Simultaneous and proportional force estimation for multifunction myoelectric prostheses using mirrored bilateral training." IEEE Transactions on Biomedical Engineering, vol. 58, no. 3, pp. 681-8, Mar. 2011.

[44] D. Tkach, H. Huang, and T. a. Kuiken, "Study of stability of time-domain features for electromyographic pattern recognition." Journal of Neuroengineering and Rehabilitation, vol. 7, p. 21, Jan. 2010.

[45] N. Jiang, K. B. Englehart, and P. a. Parker, "Extracting simultaneous and proportional neural control information for multiple-DOF prostheses from the surface electromyographic signal." IEEE Transactions on Biomedical Engineering, vol. 56, no. 4, pp. 1070-80, Apr. 2009. 
[46] S. Muceli and D. Farina, "Simultaneous and proportional estimation of hand kinematics from EMG during mirrored movements at multiple degrees-offreedom." IEEE Transactions on Neural Systems and Rehabilitation Engineering, vol. 20, no. 3, pp. 371-8, May 2012.

[47] E. Scheme and K. Englehart, "Electromyogram pattern recognition for control of powered upper-limb prostheses: State of the art and challenges for clinical use," The Journal of Rehabilitation Research and Development, vol. 48, no. 6, pp. 643-660, 2011.

[48] T. Tommasi, F. Orabona, C. Castellini, and B. Caputo, "Improving Control of Dexterous Hand Prostheses Using Adaptive Learning," IEEE Transactions on Robotics, vol. 29, no. 1, pp. 207-219, 2012.

[49] T. Lorrain, N. Jiang, and D. Farina, "Influence of the training set on the accuracy of surface EMG classification in dynamic contractions for the control of multifunction prostheses." Journal of Neuroengineering and Rehabilitation, vol. 8, no. 25, pp. 1-9, Jan. 2011.

[50] J. C. Rosenvang and R. Horup, Towards Intuitive Control of Simultaneous Movements and Force Estimation for Myoelectric Prostheses. Master Thesis, Aalborg University: Biomedical Engineering and Informatics, 2012.

[51] I. Kuzborskij, A. Gijsberts, and B. Caputo, "On the challenge of classifying 52 hand movements from surface electromyography." Conference Proceedings of the Annual International Conference of the IEEE Engineering in Medicine and Biology Society., vol. 2012, pp. 4931-7, Aug. 2012.

[52] E. J. Scheme, B. S. Hudgins, and K. B. Englehart, "Confidence-based rejection for improved pattern recognition myoelectric control." IEEE Transactions on Biomedical Engineering, vol. 60, no. 6, pp. 1563-70, Jun. 2013.

[53] E. Scheme, B. Lock, L. Hargrove, W. Hill, U. Kuraganti, and K. Englehart, "Motion Normalized Proportional Control for Improved Pattern Recognition Based Myoelectric Control." IEEE Transactions on Neural Systems and Rehabilitation Engineering, no. c, Mar. 2013.

[54] E. H. Moore, "On the reciprocal of the general algebraic matrix," Bulletin of the American Mathematical Society, vol. 26, pp. 394 - 395, 1920. 
[55] J. M. Hahne, F. Biebmann, N. Jiang, H. Rehbaum, D. Farina, F. C. Meinecke, K.-R. Muller, and L. C. Parra, "Linear and nonlinear regression techniques for simultaneous and proportional myoelectric control." IEEE Transactions on Neural Systems and Rehabilitation Engineering, vol. 22, no. 2, pp. 269-79, Mar. 2014.

[56] A. Gijsberts, R. Bohra, D. Sierra González, A. Werner, M. Nowak, B. Caputo, M. Roa, and C. Castellini, "Stable myoelectric control of a hand prosthesis using non-linear incremental learning." Frontiers in Neurorobotics, vol. 8, no. February, p. 8, Jan. 2014.

[57] D. D. Lee and H. S. Seung, "Learning the parts of objects by non-negative matrix factorization." Nature, vol. 401, no. 6755, pp. 788-91, Oct. 1999.

[58] N. Jiang, I. Vujaklija, H. Rehbaum, S. Member, and B. Graimann, "Is Accurate Mapping of EMG Signals on Kinematics Needed for Precise Online Myoelectric Control?" IEEE Transactions on Neural Systems and Rehabilitation Engineering, no. [Epub ahead of print], 2013.

[59] A. Ameri, E. Kamavuako, E. Scheme, K. Englehart, and P. Parker, "Support Vector Regression for Improved Real-Time, Simultaneous Myoelectric Control." IEEE Transactions on Neural Systems and Rehabilitation Engineering, no. [epub ahead of print], May 2014.

[60] N. Jiang, H. Rehbaum, I. Vujaklija, B. Graimann, and D. Farina, "Intuitive, Online, Simultaneous and Proportional Myoelectric Control Over Two Degrees of Freedom in Upper Limb Amputees." IEEE Transactions on Neural Systems and Rehabilitation Engineering, no. c, pp. 1-10, Aug. 2013.

[61] "COAPT Complete Control," 2014. [Online]. Available: http://www. coaptengineering.com/

[62] M. Ortiz-Catalan, R. Brånemark, and B. Håkansson, "BioPatRec: A modular research platform for the control of artificial limbs based on pattern recognition algorithms." Source Code for Biology and Medicine, vol. 8, no. 1, p. 11, Jan. 2013. 
[63] B. A. Lock, K. Englehart, D. Ph, and B. Hudgins, "Real-time myoelectric control in a virtual environment to relate usability vs. accuracy," in MyoElectric Controls Symposium, 2005, pp. 17-20.

[64] E. Scheme, A. Fougner, O. Stavdahl, a. C. Chan, and K. Englehart, "Examining the adverse effects of limb position on pattern recognition based myoelectric control." Conference Proceedings of the Annual International Conference of the IEEE Engineering in Medicine and Biology Society., vol. 2010, pp. 6337-40, Jan. 2010.

[65] E. Scheme, K. Biron, and K. Englehart, "Improving myoelectric pattern recognition positional robustness using advanced training protocols." Conference Proceedings of the Annual International Conference of the IEEE Engineering in Medicine and Biology Society., vol. 2011, pp. 4828-31, Jan. 2011.

[66] Y. Geng, P. Zhou, and G. Li, "Toward attenuating the impact of arm positions on electromyography pattern-recognition based motion classification in transradial amputees." Journal of Neuroengineering and Rehabilitation, vol. 9, no. 74, pp. 1-23, Oct. 2012.

[67] A. Fougner, E. Scheme, A. Chan, K. Englehart, and O. Stavdahl, "Resolving the Limb Position Effect in Myoelectric Pattern Recognition." IEEE Transactions on Neural Systems and Rehabilitation Engineering, vol. 19, no. 6, pp. 644-651, Aug. 2011.

[68] J. Liu, D. Zhang, X. Sheng, and X. Zhu, "Quantification and solutions of arm movements effect on sEMG pattern recognition," Biomedical Signal Processing and Control, vol. 13, pp. 189-197, Sep. 2014.

[69] A. Boschmann, B. Nofen, and M. Platzner, "Improving transient state myoelectric signal recognition in hand movement classification using gyroscopes." Conference Proceedings of the Annual International Conference of the IEEE Engineering in Medicine and Biology Society., vol. 2013, pp. 6035-8, Jan. 2013.

[70] C. Cipriani, R. Sassu, M. Controzzi, and M. C. Carrozza, "Influence of the weight actions of the hand prosthesis on the performance of pattern recognition based myoelectric control: preliminary study." Conference Proceedings of 
the Annual International Conference of the IEEE Engineering in Medicine and Biology Society., vol. 2011, pp. 1620-3, Jan. 2011.

[71] A. Gijsberts, M. Atzori, C. Castellini, H. Muller, and B. Caputo, "The Movement Error Rate for Evaluation of Machine Learning Methods for sEMG-based Hand Movement Classification," IEEE Transactions on Neural Systems and Rehabilitation Engineering, vol. 4320, no. c, pp. 1-1, 2014.

[72] A. Fougner, E. Scheme, A. D. C. Chan, K. Englehart, and O. Stavdahl, "A multi-modal approach for hand motion classification using surface EMG and accelerometers." Conference Proceedings of the Annual International Conference of the IEEE Engineering in Medicine and Biology Society., vol. 2011, no. Grant 192546, pp. 4247-50, Jan. 2011.

[73] A. Boschmann and M. Platzner, "Reducing classification accuracy degradation of pattern recognition based myoelectric control caused by electrode shift using a high density electrode array." Conference Proceedings of the Annual International Conference of the IEEE Engineering in Medicine and Biology Society., vol. 2012, pp. 4324-7, Jan. 2012.

[74] A. J. Young, L. J. Hargrove, and T. A. Kuiken, "The effects of electrode size and orientation on the sensitivity of myoelectric pattern recognition systems to electrode shift." IEEE Transactions on Biomedical Engineering, vol. 58, no. 9, pp. 2537-44, Sep. 2011.

[75] T. Lorrain, N. Jiang, and D. Farina, "Surface EMG classification during dynamic contractions for multifunction transradial prostheses." Conference Proceedings of the Annual International Conference of the IEEE Engineering in Medicine and Biology Society., vol. 2010, pp. 2766-9, Jan. 2010.

[76] E. Scheme and K. Englehart, "Training Strategies for Mitigating the Effect of Proportional Control on Classification in Pattern Recognition Based Myoelectric Control," Journal of Prosthetics \& Orthotics, vol. 25, no. 2, pp. 76-83, 2013.

[77] B. Wan, L. Xu, Y. Ren, L. Wang, S. Qiu, X. Liu, X. Liu, H. Qi, D. Ming, and W. Wang, "Study on Fatigue Feature from Forearm SEMG Signal Based on 
Wavelet Analysis," Proceedings of the 2010 IEEE International Conference of Robotics and Biomimetics, pp. 1229-1232, 2010.

[78] H. Yokoi, A. H. Arieta, R. Katoh, and W. Yu, "Mutual Adaptation in a Prosthetics Application," in Embodied Artificial Intelligence, 1st ed., M. M. Yokoi Horoshi, Arieta A, Katoh R., Wenwei Yu, Ichiro Watanabe, Ed., Berlin, 2004, ch. Mutual Ada, pp. 146-159.

[79] N. Ge, P. M. Goebel, S. Amsuess, L. Paredes, R. Pawlik, and D. Farina, "Evaluating Upper-Limb EMG-Prosthesis User Performance by Combining Psychometric Measures and Classification-Rates," in NER 2013 6th International IEEE EMBS Conference on Neural Engineering, 2013, pp. 359-363.

[80] P. Kaufmann, K. Englehart, and M. Platzner, "Fluctuating emg signals: investigating long-term effects of pattern matching algorithms." Conference Proceedings of the Annual International Conference of the IEEE Engineering in Medicine and Biology Society., vol. 2010, pp. 6357-60, Jan. 2010.

[81] S. Amsuess, L. P. Paredes, N. Rudigkeit, B. Graimann, M. J. Herrmann, and D. Farina, "Long Term Stability of Surface EMG Pattern Classification for Prosthetic Control," Conference Proceedings of the Annual International Conference of the IEEE Engineering in Medicine and Biology Society., pp. 3622-25, 2013.

[82] L. P. Paredes, S. Amsuess, P. M. Goebel, D. Hofmann, B. Graimann, and D. Farina, "Factors of Influence on the Reduction of EMG Classification Accuracy over Days in Controls and Amputees," Submitted to IEEE Transactions on Neural Systems and Rehabilitation Engineering, 2014.

[83] N. E. Bunderson and T. A. Kuiken, "Quantification of feature space changes with experience during electromyogram pattern recognition control." IEEE Transactions on Neural Systems and Rehabilitation Engineering, vol. 20, no. 3, pp. 239-46, May 2012.

[84] S. Amsuess, P. Gobel, N. Jiang, B. Graimann, L. Paredes, and D. Farina, "SelfCorrecting Pattern Recognition System of Surface EMG Signals for Upper Limb Prosthesis Control," IEEE Transactions on Biomedical Engineering, vol. 61, no. 4, pp. 1167-76, 2014. 
[85] M. M. Vidovic, L. P. Paredes, H.-J. Hwang, S. Amsuess, J. Pahl, J. M. Hahne, B. Graimann, D. Farina, and K.-R. Mueller, "Covariate Shift Adaptation in EMG Pattern Recognition for Prosthetic Device Control," Conference Proceedings of the Annual International Conference of the IEEE Engineering in Medicine and Biology Society., 2014.

[86] S. Amsuess, P. Goebel, H. Rehbaum, and R. Pawlik, "Applying LDA to Accomplish Simultaneous 2-DOF Movements using Visual Feedback," in Conference Proceedings of the Annual International Conference of the IEEE Engineering in Medicine and Biology Society., San Diego, CA, USA, 2012.

[87] L. H. Smith, L. J. Hargrove, B. A. Lock, and T. A. Kuiken, "Determining the optimal window length for pattern recognition-based myoelectric control: balancing the competing effects of classification error and controller delay." IEEE Transactions on Neural Systems and Rehabilitation Engineering, vol. 19, no. 2, pp. 186-92, Apr. 2011.

[88] S. M. Wurth and L. J. Hargrove, "A real-time comparison between direct control, sequential pattern recognition control and simultaneous pattern recognition control using a Fitts' law style assessment procedure." Journal of Neuroengineering and Rehabilitation, vol. 11, no. 91, pp. 1-7, May 2014.

[89] J. W. Tukey, "The Philosophy of Multiple Comparisons," Statistical Science, vol. 6, no. 1, pp. 100-116, 1991.

[90] C. Y. Kramer, "Extension of Multiple Range Tests to Group Means with Unequal Numbers of Replications," International Biometric Society, vol. 12, no. 3, pp. 307-310, 1956.

[91] T. R. Farrell and R. F. Weir, "The optimal controller delay for myoelectric prostheses." IEEE Transactions on Neural Systems and Rehabilitation Engineering, vol. 15, no. 1, pp. 111-8, Mar. 2007.

[92] J. W. Sensinger, B. Lock, and T. Kuiken, "Adaptive pattern recognition of myoelectric signals: exploration of conceptual framework and practical algorithms." IEEE Transactions on Neural Systems and Rehabilitation Engineering, vol. 17, no. 3, pp. 270-8, Jun. 2009. 
[93] J. M. Hahne, B. Graimann, and K.-R. Müller, "Spatial filtering for robust myoelectric control." IEEE Transactions on Biomedical Engineering, vol. 59, no. 5, pp. 1436-43, May 2012.

[94] S. Amsuess, P. Goebel, B. Graimann, and D. Farina, "A Novel Multi-Class Proportional Myocontrol Algorithm for Upper Limb Prosthesis Control: Validation in Real-Life Scenarios on Amputees," Submitted to IEEE Transactions on Neural Systems and Rehabilitation Engineering, pp. 1-10, 2014.

[95] — - "Extending mode switching to multiple degrees of freedom in hand prosthesis control is not efficient," Conference Proceedings of the Annual International Conference of the IEEE Engineering in Medicine and Biology Society., 2014.

[96] Z. J. Koles, "The quantitative extraction and topographic mapping of the abnormal components in the clinical EEG." Electroencephalography and Clinical Neurophysiology, vol. 79, no. 6, pp. 440-7, Dec. 1991.

[97] J. Müller-Gerking, G. Pfurtscheller, and H. Flyvbjerg, "Designing optimal spatial filters for single-trial EEG classification in a movement task," Clinical Neurophysiology, vol. 110, no. 5, pp. 787-798, 1999.

[98] H. Ramoser, J. Müller-Gerking, and G. Pfurtscheller, "Optimal spatial filtering of single trial EEG during imagined hand movement." IEEE Transactions on Rehabilitation Engineering, vol. 8, no. 4, pp. 441-6, Dec. 2000.

[99] B. Blankertz, M. Kawanabe, R. Tomioka, F. Hohlefeld, V. Nikulin, and K.-R. Müller, "Invariant Common Spatial Patterns: Alleviating Nonstationarities in Brain-Computer Interfacing." in NIPS, 2008, pp. 1-8.

[100] F. Lotte and C. Guan, "Regularizing common spatial patterns to improve BCI designs: unified theory and new algorithms." IEEE Transactions on Biomedical Engineering, vol. 58, no. 2, pp. 355-62, Feb. 2011.

[101] W. Samek, C. Vidaurre, K.-R. Müller, and M. Kawanabe, "Stationary common spatial patterns for brain-computer interfacing." Journal of Neural Engineering, vol. 9, no. 2, p. 026013, Apr. 2012. 
[102] F. S. Cromwell, Occupational therapist's manual for basic skills assessment or primary pre-vocational evaluation, 1st ed. Pasadena: Fair Oaks Printing Co., 1960.

[103] V. Mathiowetz, G. Volland, N. Kashman, and K. Weber, "Adult Norms for the Box and Block Test of Manual Dexterity," The American Journal of Occupational Therapy, vol. 39, no. 6, pp. 386-391, 1985.

[104] V. Mathiowetz, S. Federman, and D. Wiemer, "Box and Block Test of Manual Dexterity: Norms for 6-19 Year Olds," Canadian Journal of Occupational Therapy, vol. 52, no. 5, pp. 241-245, 2004.

[105] T. A. Kuiken, G. A. Dumanian, R. D. Lipschutz, L. A. Miller, and K. A. Stubblefield, "The use of targeted muscle reinnervation for improved myoelectric prosthesis control in a bilateral shoulder disarticulation amputee," Prosthetics and Orthotics International, vol. 28, no. 3, pp. 245-253, 2004.

[106] "Patterson Medical." [Online]. Available: http://www.pattersonmedical.com

[107] W. Hill, P. Kyberd, L. Norling Hermansson, S. Hubbard, O. y. Stavdahl, and S. Swanson, "Upper Limb Prosthetic Outcome Measures (ULPOM): A Working Group and Their Findings," JPO Journal of Prosthetics and Orthotics, vol. 21, no. Supplement, pp. 69-82, Oct. 2009.

[108] P. J. Kyberd and W. Hill, "Survey of upper limb prosthesis users in Sweden, the United Kingdom and Canada." Prosthetics and Orthotics International, vol. 35, no. 2, pp. 234-41, Jun. 2011.

[109] K. Ø stlie, I. M. Lesjø, R. J. Franklin, B. Garfelt, O. H. Skjeldal, and P. Magnus, "Prosthesis rejection in acquired major upper-limb amputees: a populationbased survey." Disability and Rehabilitation. Assistive Technology, vol. 7, no. 4, pp. 294-303, Jul. 2012.

[110] N. Jiang, "Extracting force functions from the multi-channel surface electromyographic signal," Ph.D. dissertation, The University of New Brunswick, 2008.

[111] M. Markou and S. Singh, "Novelty Detection: A ReviewPart 1: Statistical Approaches," Signal Processing, vol. 83, no. 12, pp. 2499-2521, Dec. 2003. 
[112] B. Schoelkopf, R. Williamson, A. Smola, J. Shawe-taylor, and J. Platt, "Support Vector Method for Novelty Detection," NIPS, vol. 12, pp. 582-588, 1999.

[113] C. Chih-Chung and L. Chih-Jen, "Lib-SVM." [Online]. Available: http: //www.csie.ntu.edu.tw/ cjlin/libsvm/

[114] P. Bodesheim, A. Freytag, E. Rodner, M. Kemmler, and J. Denzler, "Kernel Null Space Methods for Novelty Detection," 2013 IEEE Conference on Computer Vision and Pattern Recognition, pp. 3374-3381, Jun. 2013.

[115] P. Bodesheim, A. Freytag, E. Rodner, K. Michael, and J. Denzler, "KNFST Matlab code," 2013. [Online]. Available: http://www.inf-cv.uni-jena.de/ dbvmedia/source_code/code_knfst.zip

[116] S. Amsuess, "Assignment of statistical analysis course in R (unpublished)," 2014.

[117] S. Holm, "A simple sequentially rejective Bonferroni test procedure," Scandinavian Journal on Statistics, vol. 6, pp. 65-70, 1979.

[118] C. M. Light, P. H. Chappell, and P. J. Kyberd, "Establishing a standardized clinical assessment tool of pathologic and prosthetic hand function: Normative data, reliability, and validity," Archives of Physical Medicine and Rehabilitation, vol. 83, no. 6, pp. 776-783, Jun. 2002.

[119] S. Dalley, D. Bennett, and M. Goldfarb, "Preliminary functional assessment of a multigrasp myoelectric prosthesis." Conference Proceedings of the Annual International Conference of the IEEE Engineering in Medicine and Biology Society., vol. 2012, pp. 4172-5, Aug. 2012.

[120] M. M. D. Sobuh, L. P. J. Kenney, A. J. Galpin, S. B. Thies, J. McLaughlin, J. Kulkarni, and P. Kyberd, "Visuomotor behaviours when using a myoelectric prosthesis." Journal of Neuroengineering and Rehabilitation, vol. 11, no. 1, p. 72, Jan. 2014.

[121] J. A. Adams, "Historical review and appraisal of research on the learning, retention, and transfer of human motor skills." Psychological Bulletin, vol. 101, no. 1, pp. 41-74, 1987. 
[122] A. Phinyomark, P. Phukpattaranont, and C. Limsakul, "Investigating LongTerm Effects of Feature Extraction Methods for Continuous Emg Pattern Classification," Fluctuation and Noise Letters, vol. 11, no. 04, p. 1250028, Dec. 2012. 


\section{$7 \quad$ Supplement}

\subsection{List of publications}

1. Amsüss, S., Goebel, P.M., Ning Jiang, Graimann, B., Paredes, L., Farina, D., "Self-Correcting Pattern Recognition System of Surface EMG Signals for Upper Limb Prosthesis Control", IEEE Transactions on Biomedical Engineering, vol.61, no.4, pp.1167-1176, April 2014

2. Amsüss, S., Goebel, P., Graimann, B., Farina, D., "A Multi-Class Proportional Myocontrol Algorithm for Upper Limb Prosthesis Control: Validation in Real-Life Scenarios on Amputees", submitted to IEEE Transactions Of Transactions On Neural Systems And Rehabilitation Engineering.

3. Amsüss, S., Paredes, L.P., Rudigkeit, N., Graimann, B., Herrmann, M.J., Farina, D., "Long term stability of surface EMG pattern classification for prosthetic control," Engineering in Medicine and Biology Society (EMBC), 2013, 35th Annual International Conference of the IEEE, pp.3622-25

4. Amsüss, S., Goebel, P.M., Pawlik, R., "Applying LDA to Accomplish Simultaneous 2-DOF Movements using Visual Feedback", Engineering in Medicine and Biology Society (EMBC), 2012, 34th Annual International Conference of the IEEE, 1 page paper (poster presentation)

5. Amsüss, S., Goebel P, Graimann, B., Farina, D., "Simultaneous, Proportional Wrist And Hand Control For Natural, Dexterous Movements Of A Physical Prosthesis By Amputees", 2014, Myoelectric Control Symposium (MEC).

6. Amsüss, S., Goebel P., Graimann, B., Farina, D., "Extending mode switching to multiple degrees of freedom in hand prosthesis control is not efficient", Engineering in Medicine and Biology Society (EMBC), 2014, 36th Annual International Conference of the IEEE. 
7. Amsüss, S., Roche A.D., Goebel P., Graimann B., Farina D., Aszmann O.C., "Regaining High Functional, Multiple Degrees Of Freedom Hand Control Following Bionic Reconstruction", Peripheral Nervous System Machine Interface (PNS-MI) Workshop held at the Myoelectric Control Symposium (MEC) 2014.

8. Hartmann, C., Dosen, S., Amsüss, S., Farina, D., "Closed-Loop Control of Myoelectric Prostheses with Electrotactile Feedback: Inuence of Stimulation Artifact and Blanking", submitted to IEEE Transactions Of Transactions On Neural Systems And Rehabilitation Engineering.

9. Nan Ge, Goebel, P.M., Amsüss, S., Paredes, L., Pawlik, R., Farina, D., "Evaluating upper-limb EMG-prosthesis user performance by combining psychometric measures and classification-rates," Neural Engineering (NER), 2013, 6th International IEEE/EMBS Conference, pp.359-362

10. Rehbaum, H., Ning Jiang, Paredes, L., Amsüss, S., Graimann, B., Farina, D., "Real time simultaneous and proportional control of multiple degrees of freedom from surface EMG: Preliminary results on subjects with limb deficiency," Engineering in Medicine and Biology Society (EMBC), 2012, 34th Annual International Conference of the IEEE, pp.1346-1349,

11. Vidovic M., Paredes LP., Pahl J., Amsüss, S., Hwang H.J., Graimann B., Müller K.R., "Covariate Shift Adaptation in EMG Pattern Recognition for Prosthetic Device Control", Engineering in Medicine and Biology Society (EMBC), 2014, 36th Annual International Conference of the IEEE.

The podium presentation "Simultaneous, Proportional Wrist And Hand Control For Natural, Dexterous Movements Of A Physical Prosthesis By Amputees" (presenting the final system of this thesis), held at the MEC Symposium 2014, was awarded the $1^{\text {st }}$ prize as Best Student Paper presentation of that conference.

\subsection{Reviewer activities}

During my Ph.D. studies, I have served as a reviewer for a total of 12 papers submitted for journal publication to IEEE Transactions of Neural Systems and Rehabilitation Engineering (5), IEEE Transactions of Biomedical Engineering (5), Journal of Electromyography \& Kinesiology (1), Frontiers in Computational Neuroscience (1) and 3 conference papers (Myoelectric Control Symposium 2014, Fredericton, Canada). 
Sebastian Amsüss

Ginsterweg 18/310

37077 Göttingen, Germany

Mobile: 0176/47753346

E-mail: sebastian.amsuess@gmail.com

Date of birth: February 1 1985, Klosterneuburg, AUT

Marital status: Single

Nationality: Austrian

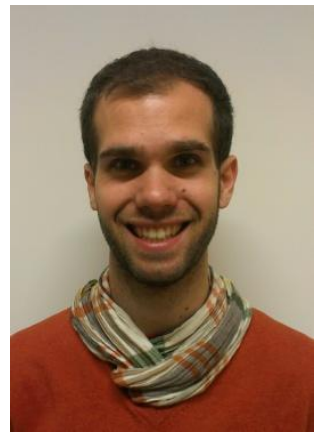

\section{Education}

1995 - 2003 High School Bundesrealgymnasium XVIII Schopenhauerstraße, Vienna Graduation with „Excellent Success“

2005 - 2008 Bachelor of Science, Biomedical Engineering, UAS Technikum Vienna $5^{\text {th }}$ semester at University of Vigo, Spain

2 Bachelor theses:

- Socket types for hand prostheses

- Development of rehabilitation device after nerve transsection Graduation with „Very Good Success””

2008-2010 Master of Science, Healthcare- and Rehabilitation Technology, UAS Technikum Vienna Master thesis:

- Virtual Sensibility for peripheral nerve injury rehabilitation Graduation with „Excellent Success”

\section{Professional Experience}

$2004-2005$ Civilian service, Johanniter Ambulance, Vienna

First aid in medical emergencies as paramedic, ambulance service. Stayed for two more years as volunteer.

$2007-2011$ Master thesis and scientific staff, Ludwig Boltzmann Institute for clinical and experimental traumatology, Vienna

- Development of a rehabilitation device after nerve injuries to the hand

- Recording and evaluation of $\mu C T$ images for preclinical studies

2011 - 2012 Software developer, Otto Bock Healthcare Products GmbH, Vienna Research and development: Hand prosthesis control through pattern recognition of EMG Signals

2013 - act. PhD. Student and scientific staff, Medical University Center Göttingen, Department for Neurorehabilitation Engineering Research on hand prosthesis control through machine learning applied to EMG signals as Marie-Curie fellow in EU IAPP project „AMYO“ 


\section{Further interests}

$\begin{array}{lll}\text { Languages } & \begin{array}{l}\text { German } \\ \text { English } \\ \text { French } \\ \text { Spanish }\end{array} & \begin{array}{l}\text { Native speaker } \\ \text { Fluent }\end{array} \\ & \begin{array}{l}\text { Very good command } \\ \text { Very good command }\end{array} \\ \text { Computer } & \begin{array}{l}\text { Operating system } \\ \text { Documentation }\end{array} & \begin{array}{l}\text { Microsoft Windows } \\ \text { Microsoft Office, Latex }\end{array} \\ & \text { Programming languages } & \text { Visual C\#, Matlab. Java for Android, Arduino } \\ \text { Hobbies } & \text { Biking } & \text { Trial, Mountainbike } \\ & \text { Electronics } & \text { Projects based on Arduino platform } \\ & \text { Software } & \text { Java for Android (self study) } \\ \text { Languages } & \text { French and Spanish in self study } \\ & \text { Various } & \text { Cooking, rock climbing, pool billiard }\end{array}$

Göttingen, August $27^{\text {th }} 2014$

Sebastian Amsüss 